\title{
HISTOIRE ET TERRITOIRES DES SCIENCES SOCIALES
}

Le Monde végétal (xIf-xvII siècles). Savoirs et usages sociaux. Sous la dir. d'Allen J. Grieco, Odile Redon, Lucia Tongiorgi Tomasi. Saint-Denis, Presses universitaires de Vincennes, 1993. 13,5 × 22, 188 p., index (« Essais et savoirs »).

La présentation indique que ce volume rassemble les actes d'un colloque qui s'est tenu à Florence en 1989, "promu par le Harvard University Center for Italian Renaissance Studies, Villa i Tatti à Florence, l'Institut culturel français de Florence, l'Institut universitaire européen de Florence et l'Université de Paris-VIII-Vincennes à Saint-Denis ». L'abondance des patronages contraste avec le nombre relativement réduit des contributions rassemblées : cinq articles en français et quatre en italien (avec résumés français). Mais la concision et la sobriété vont ici de pair avec la maniabilité d'autant qu'un index et des illustrations bien choisies contribuent à faciliter la consultation. Le bilinguisme du colloque et de ce recueil est également à saluer. Enfin le thème des rapports entre le monde végétal et les sociétés humaines, qui constitue l'objet propre de l'ethnobotanique, a été relativement peu traité jusqu'ici dans l'historiographie, ce qui rend d'autant plus utile ce petit volume.

Les contributions sont regroupées en deux parties. La première, intitulée « Du bon usage des plantes », est composée de quatre contributions. La consommation alimentaire directe des végétaux y tient la plus grande place puisque Allen J. Grieco traite de la signification symbolique des régimes végétariens à la fin du Moyen Âge (p. 11-29), Bernard Rosenberger, des « recettes pour les temps de disette au Maroc » (p. 31-58), et Jean-Louis Flandrin des «légumes dans les livres de cuisine français du XIv ${ }^{\mathrm{e}}$ au XVIII ${ }^{\mathrm{e}}$ siècle » (p. 71-85). Toutefois le texte de Mauro Ambrosoli, «Erbe selvatiche et leguminose nel lungo periodo: il caso delle Medicago prima del 1600 », ménage une ouverture particulièrement heureuse sur l'histoire de l'agriculture et de l'élevage (p. 59-70). On regrettera d'autant plus que Medicago sativa, l' « erba medica » du texte italien, devienne le « sainfoin » dans le résumé français, alors que l'auteur de l'article explique qu'il s'agit de la luzerne et que « sainfoin" qui, au XvI siècle était synonyme de luzerne, désigne aujourd'hui en français la plante dont le nom italien est « lupinella » (Onobrychis sativa).

Sous le titre "Science et rêve du végétal », la deuxième partie rassemble cinq contributions. L'étude de Lucia Tongiorgi Tomasi sur le paysage et les illustrations botaniques dans l'œuvre d'un peintre italien du xvie siècle (Gherardo Cibo) et celle

Revue de synthèse : $4^{e} \mathrm{~S}$. $\mathrm{N}^{\mathrm{o}}$ 4, oct.-déc. 1997, p. 551-613. 
de Bruno Laurioux sur « l'imaginaire médiéval des épices » manifestent l'apport de l'histoire de l'art et de l'histoire culturelle (p. 107-122 et p. 157-174). Le texte de Laurence Moulinier, sur Hildegarde de Bingen et Albert le Grand (p. 89-105), et celui de Guiddo Moggi, « La conoscenza del mondo vegetale prima e doppo Andrea Cesalpino " (p. 123-140), se complètent pour donner une vue panoramique de la botanique savante au Moyen Âge et à la Renaissance. On appréciera en particulier les documents qui accompagnent l'étude sur Cesalpino (p. 135 sq.). C'est à la botanique aussi mais surtout à l'horticulture qu'est consacré l'article de Milena Rizotto sur les pratiques de sélection au $x{ }^{\mathrm{e}}$ siècle : «Le amene monstruosità : coltora, selezione e manipolazione delle piante ornementali nel xVII secolo» (p. 141-155). Cet article combine information scientifique et érudition historique en une synthèse du plus grand intérêt pour l'historien des sciences. Comme l'ont noté les auteurs qui ont écrit sur l'origine des plantes cultivées, les conditions particulières créées par la culture permettent à des variations, intéressantes pour l'homme mais désavantageuses pour la plante, de subsister. Cela est vrai en particulier pour les plantes ornementales, ces «monstres agréables» selon l'expression employée par Milena Rizotto. Or le statut de ces monstruosités a joué un rôle important - au-delà de la période considérée ici - dans l'étude de la morphologie végétale.

En définitive, les limites de cet ouvrage tiennent à ses mérites. Limité au bas Moyen Âge et au début des temps modernes, centré sur l'espace occidental et méditerranéen, il évite les difficultés qu'entraîne un trop grand éloignement spatial ou temporel, sans avoir à traiter des développements scientifiques du xviI ${ }^{\mathrm{e}}$ siècle. $\grave{A}$ l'intérieur de ce cadre, il ne prétend à aucune exhaustivité et livre des études partielles mais suggestives. Il juxtapose des approches un peu disparates mais révèle ainsi la variété des disciplines concernées.

Au total donc, un livre stimulant, qui instruit et qui donne à penser. Il reste à espérer qu'il suscite d'autres rencontres et inspire d'autres travaux dans le même champ de recherche.

Jean-Marc Drouin

Eustache Delafosse, Voyage sur la côte de Guinée, au Portugal \& en Espagne (1479-1481). Transcription du manuscrit de Valenciennes, trad. et prés. de Denis Escudier, av.-pr. de Théodore MonOD. Paris, Chandeigne/Librairie portugaise, 1992. 15,6 × 22, 183 p., index, bibliogr. ( Collection Magellane »).

Entre 1479 et 1481, Eustache Delafosse, facteur d'une maison de commerce de Bruges, fut envoyé « à la Mine d'Or », c'est-à-dire sur la côte de Guinée, afin de se procurer par troc l'or et l'épice (la malaguette). Or les Portugais avaient établi là un monopole qu'ils entendaient faire respecter. Arrêté, le marchand fut conduit au Portugal. Menacé d'être pendu, il s'évada, gagna la Castille puis les Asturies, où il s'embarqua pour Bruges. Une dizaine d'années plus tard sans doute, il rédigea en français le récit de ses aventures.

On en trouve ici l'édition et la traduction en français moderne, l'une et l'autre présentées en vis-à-vis. Texte unique, non pas par son objet, car on possède d'autres 
textes contemporains, mais par sa langue : pour plusieurs décennies, c'est le seul récit de voyage en français vers les zones nouvellement découvertes.

L'auteur témoigne d'une fraîcheur de vision et d'expression qui renseigne sur la psychologie du marchand. Plus qu'aux hommes, il s'intéresse aux langues africaines, aux techniques de navigation, aux marchandises, à l'île du Cap-Vert où les lépreux cherchent la guérison par l'immersion dans le sang de tortue, espoir qui séduira Louis XI à la veille de sa mort... Sensible au merveilleux, il s'attarde sur les îles mystérieuses où abordèrent autrefois des fidèles fuyant l'invasion musulmane, et qui ne réapparaitront que lorsque toute l'Espagne sera revenue à la foi catholique.

Cette brève relation d'une trentaine de pages introduit le lecteur au moment psychologique des Découvertes. C'est tout le mérite de Denis Escudier de porter ensuite avec science et élégance les éclairages nécessaires sur le manuscrit, l'auteur et les circonstances.

L'ouvrage se clôt par une chronologie du voyage, un index des noms propres et une abondante bibliographie. Il est opportun de souligner, enfin, que la typographie, le papier et l'impression, par leur qualité, font de ce petit ouvrage une exception remarquable dans la production éditoriale courante.

Patrick Gautier Dalché

Antonio R. Damasio, L'Erreur de Descartes. La raison des émotions. Trad. de l'anglais (États-Unis) par Marcel BLANC. Paris, Odile Jacob, 1995. $15 \times 24$, 365 p., index.

L'ouvrage d'Antonio Damasio ne peut être réduit à sa seule fonction vulgarisatrice. L'ambition de ce travail, avouée dès les premières pages, est d'opérer un retour sur les «conceptions traditionnelles » du raisonnement, à partir des développements les plus récents de l'analyse des mécanismes neuraux. Ce retour s'effectue en plusieurs temps : aux traditionnelles, et très anglo-saxonnes, études empiriques de cas succèdent le passage en revue des découvertes en neuropsychologie animale et humaine, puis l'énoncé de l'hypothèse de travail selon laquelle la faculté de raisonner (la généralité de cette notion est passablement gênante) dépend de systèmes de neurones agissant de conserve à différents niveaux de l'organisation neurale. Le « haut niveau » des explications traditionnelles (qui confèrent au seul centre cérébral la puissance de raisonner) se voit secondé par le «bas niveau " corporel. Dans le raisonnement se trouvent impliqués les mêmes mécanismes que ceux dont dépendent les émotions ainsi que les fonctions corporelles inconscientes liées à la survie. Là est, selon l'auteur, l'idée décisive : raisonner, c'est employer le corps tout autant que cet antique " esprit » dont, à la suite de Ryle, il dénonce logiquement le caractère d'entité impalpable. L'entreprise est passionnante puisqu'elle donne au conflit entre thèses réalistes de l'âme (voir les homoncules raillés par Freud ou Sartre) et thèses réfutant l'existence de cette âme, sa traduction neurologique : il s'agit de savoir si la perception des émotions (second thème cher à Damasio, et qui suit logiquement sa thèse concernant la faculté de raisonner) ne se situe que dans le 
système limbique, ou bien s'il faut y adjoindre certaines parties du cortex préfrontal, ainsi que certaines régions du cerveau où se recueillent les signaux émis par le corps. Ce second thème (la perception des émotions « est un état du corps » et a une valeur cognitive, p. 11) uni au premier (le raisonnement n'est pas une activité pure) achève l'architectonique conceptuelle de cet ouvrage : la perception des émotions est au fondement de ce qu'une analyse traditionnelle nomme « âme " ou " esprit ». Le corps constitue, à travers la mise en évidence du rôle du bas niveau, émotionnel, de l'organisation neurologique dans le fonctionnement de l'ensemble, le cadre de référence nécessaire des processus neuraux.

Le projet de cet ouvrage est à la fois très riche et très décevant.

Très riche car il fait entrevoir, chez un grand neurologue, la tentative d'éprouver une intuition philosophique prometteuse : la nécessité de re-penser la place du corps dans l'enchevêtrement des découvertes positives en neurosciences. Cette résonance philosophique le différencie assurément de bien d'autres ouvrages qui couvrent, pour un large public, le même champ. On songe ici à Évolution du cerveau et création de la conscience de John Eccles (Paris, Flammarion, 1994 pour l'édition de poche), qui possède pour ambition de justifier la création de l' «âme » avec les outils de la théorie de l'Évolution et dont l'intention est d'éradiquer le néomatérialisme induit par les avancées en neurosciences. On comprend que Damasio soit introduit aux Éditions Odile Jacob par un J.-P. Changeux plutôt que par un J. Eccles. Damasio évite par ailleurs l'écueil consistant à réduire l'analyse du comportement à la seule neurologie. Dans le chapitre iv, il plaide pour l'intégration de toutes les « variables pertinentes », au nombre desquels il compte les facteurs socioculturels du passé et du présent. Cette version humble de l'analyse neuronale rappelle, à juste titre, le statut de science humaine qui est celui de la neurologie. La tentation de délivrer une connaissance intégrale du comportement humain (ayant par exemple des applications dans la prévention ou la répression des actes de violence urbaine) est bannie, conformément à la finalité d'une « science humaine » qui n'est que d'analyser (c'est-à-dire de détruire ou de fragmenter) l'homme en l'un de ses aspects.

Très décevant, car l'ambition philosophique évidente de cet ouvrage repose sur une double méprise. La première tient dans la croyance en l'aspect révolutionnaire de cette réhabilitation du corps. Le propos est très structuré, il passe de l'analyse historique et clinique du cas de Phineas Gage (victime, au XIX ${ }^{\mathrm{e}}$ siècle, d'une lésion du cerveau ayant entraîné un bouleversement de sa faculté de décision) jusqu'à l'affirmation plus théorique selon laquelle le «moi » de la décision, de l'émotion, n'est pas un homoncule mais une élaboration mentale intégrant des processus du cerveau et la représentation constante d'un état du corps. Mais si on perçoit l'importance de cette thèse au sein d'une radicalisation cognitiviste des analyses du cerveau (oubliant, de fait, de rapporter à un corps les événements neuraux), on ne voit pas en quoi cette thèse est philosophiquement novatrice. Or c'est, par mégarde, ce que Damasio semble penser. La seconde méprise consiste, à l'instar de Ryle, à nommer Descartes grand homonculateur. La thèse selon laquelle nous aurions un accès privé aux « états de l'âme » est plutôt lockienne. Mais bien plus, il faudrait revoir ce préjugé qui veut que, lorsque l'on tient un discours qui réhabilite le corps, on commence par jeter l'anathème sur le dualisme cartésien. 
La thèse centrale de Damasio tient en l'affirmation suivante : on ne peut faire l'économie d'une référence au corps lorsque l'on prétend comprendre les processus mentaux. Cette thèse, volontairement rapprochée des travaux de Francisco Varela et de Eleanor Rosch (cités p. 297 pour The Embodied Mind, Cambridge, MA, MIT Press, 1992) ou de ceux, philosophiques, de Dennett (La Conscience expliquée, trad. Pascal Engel, Paris, Odile Jacob, 1993 - auteur dont Eccles raille les idées inspirées de Ryle et dont Damasio reprend la thèse selon laquelle «il n'y a pas de Théâtre cartésien dans notre cerveau »), est donnée par Damasio comme une façon nouvelle de comprendre les processus mentaux. Mais si l'on donne, comme le fait Damasio lui-même en bien des rencontres, un sens ouvert et non exclusivement neurologique à la notion de « mental ", l'incarnation de la pensée, l'idée même que l'on ne peut séparer la totalité organique que forment le corps et l'activité de pensée, n'est pas une idée très neuve. Elle n'est en rien " exagérée » (p. 294) et serait même plutôt banale. La psychanalyse n'est certainement, pour faire court, que la tentative de comprendre le fonctionnement d'un esprit enfoncé dans un corps singulier (songeons à la "psychologie concrète " d'un Politzer, qui se heurte à la nécessité de penser les événements du corps). Lorsque Damasio évoque lui-même (p. $291 s q$.), au titre de conséquence majeure, la nécessité de postuler deux réseaux de signaux intervenant dans la perception d'une chose extérieure (un signal transmettant « la forme et la texture de l'objet », d'une part, et d'autre part un signal destiné à l'usage interne du corps qui localise et cartographie le site de la modification d'un état du corps), il affirme que le corps «sent », qu'il "voit» ou «touche» un objet : le corps se voit, voyant. Cette réflexivité du corps, cette façon de supposer l'inséparabilité des événements du moi et des événements du corps fait irrésistiblement penser à la phénoménologie d'un Merleau-Ponty.

Plus avant encore, Damasio invente de toutes pièces un Descartes-repoussoir, figé dans le rôle d'ennemi du corps. C'est à coup sûr un contresens que d'affirmer que, chez Descartes, le corps n'est pour rien dans l'activité de pensée.

Faute d'avoir distingué les différents niveaux de conceptualisation de l'activité de pensée chez Descartes, Damasio a feint de considérer que la philosophie cartésienne tenait toute dans le dualisme qui sépare l'âme du corps. C'est le cas dans la perspective logique et ontologique du dégagement des essences. Mais le discours cartésien, sans faire droit à une psychologie authentique et anachronique (c'est peut-être ce que Damasio lui reproche), tient parfaitement compte de cette donnée anthropologique fondamentale selon laquelle la pensée de chaque individu se fait sous la dépendance de l'union de l'âme et du corps, cause des préjugés de l'enfance et de leur ténacité : l'esprit purement attentif, non diverti par les fictions imaginatives dont l'origine est corporelle, n'existe pas chez Descartes. C'est bien de cette façon qu'est déduite la possibilité de l'erreur, dans la Quatrième Méditation. Une déduction, une inférence logique ou une vérité nécessaire peuvent être analysées sans le recours à l'insertion de l'esprit dans un corps. Il y a chez Damasio, semble-t-il, confusion à nommer unilatéralement "raisonnement " ce que Descartes nomme «entendement » dans la compréhension des concepts et «jugement» dans la conduite prudente de la vie. D'autre part, la distinction réelle des substances, sur laquelle, après tant d'autres, s'attarde Damasio, fait droit, dans la Sixième Méditation, au sentiment, fondé dans l'union concrète, que ce corps est mien : « La nature m'enseigne aussi par ces sentiments de douleur, de faim, de soif, etc., que je ne suis 
pas seulement logé dans mon corps, ainsi qu'un pilote en son navire, mais, outre cela, que je lui suis conjoint très étroitement et tellement confondu et mêlé que je compose comme un seul tout avec lui ». Cette métaphore célèbre indique assez l'inadéquation de la perspective adoptée par Damasio.

Le portrait qui est fait ici de Descartes est, il faut l'avouer, d'un simplisme irritant et il convient, sur ce point, de parler de l'erreur de Damasio plutôt que de celle de Descartes. Certes, Damasio n'est pas historien de la philosophie (et rappelle, p. $16 s q$., que son livre « ne porte ni sur Descartes ni sur la philosophie ») mais il y aurait beaucoup à dire de cette façon de tirer à soi le nom et le prestige d'un philosophe, afin d'assurer la diffusion de ses propres idées. De même qu'il est assez étrange de citer Éluard d'après l'Anthologie de G. Pompidou (p. 317), il est peu banal, pour un ouvrage dont c'est le titre, de n'analyser l' « erreur» de Descartes que par des lieux communs. Il s'agit néanmoins d'un ouvrage à lire, car l'information qui s'y donne est à la fois riche en contenus de savoir et spéculativement prometteuse.

Fabien CHAREIX

Jacques Roger, Pour une histoire des sciences à part entière. Texte établi et présenté par Claude BlancKaERT, av.-pr. de $\mathbf{M}^{\text {me }}$ Marie-Louise Roger, postf. de Jean GAYon. Paris, Albin Michel, 1995. 14,5 × 22,5, 476 p., bibliogr. (« Bibliothèque Albin Michel Idées »).

Jacques Roger (1920-1990) a profondément marqué l'histoire des sciences française. Ce livre rassemble quelques-uns de ses plus importants articles, dont certains étaient inédits en français ou trop peu connus. Ils couvrent le vaste parcours de J. Roger, depuis la question de la naissance de la science moderne jusqu'aux développements de l'eugénisme et de la sociobiologie, en passant par l'étude des médecins et naturalistes des $\mathrm{XVII}^{\mathrm{e}}$ et $\mathrm{XVII}{ }^{\mathrm{e}}$ siècles, ou l'apparition et les transformations des théories de l'évolution aux $\mathrm{xIx}^{\mathrm{e}}$ et $\mathrm{xx}^{\mathrm{e}}$ siècles. Toujours très informatives, ces diverses études offrent aussi une forme de discours de la méthode en histoire des sciences.

Il faut prendre toute la mesure du titre si juste donné à ce recueil. J. Roger était un historien à part entière. Il a étudié l'histoire pour comprendre l'histoire; c'est-à-dire pour tenter de décrire la différence du passé et expliquer le mouvement de sa transformation. L'histoire des sciences vise alors, à la fois, l'étude du " passé dans son originalité irréductible» (p.51), et l'analyse des continuités qui permettent de comprendre les «transformations profondes de la manière dont l'homme saisit le monde » (p. 96). La difficile dialectique entre ces deux exigences fut un axe central du travail de J. Roger.

D'une part, l'historien ne doit pas poser l'existence de lois ou de cadres normatifs qui surplomberaient son objet. La science ne peut s'expliquer simplement par la découverte progressive d'une connaissance vraie de la nature à travers les « obstacles épistémologiques ». Elle ne peut se résumer ni à des contraintes idéologiques, 
sociologiques ou institutionnelles, ni à une logique intrinsèque des concepts : « [...] les voies de l'histoire des sciences ne sont pas toujours aussi directes et logiques que le philosophe pourrait le souhaiter » (p. 261). L'historien étudie des hommes, dans toute la complexité de leurs comportements, de leurs sociétés, de leurs idées et de leurs traditions. Il doit respecter l'altérité du passé et tenter de dégager les « attitudes fondamentales " (p. 97) devant la nature qui marquent une époque ou un groupe social. L'invention ou le succès d'une nouvelle façon de voir les choses doivent se comprendre dans l'histoire, et par l'histoire.

Mais, d'autre part, si cette étude « des structures profondes de la sensibilité et de l'imagination » (p. 19) était proche de celle des « episteme » de Michel Foucault ou de celle des « paradigmes » de Thomas Kuhn (quand la notion est employée dans sa plus grande généralité), J. Roger se refusait à en conclure à un discontinuisme abrupt, une incommensurabilité soudaine entre paradigmes : "On passe du rien au tout, de l'éternité à l'instant présent, sans qu'aucune épaisseur de temps s'interpose, sans qu'aucune durée permette une évolution, serve de lieu à une histoire » (p. 231). Une telle option résulte de simplifications exagérées qui visent à présenter chaque époque par une structure intellectuelle et sociale complète et stable. Au contraire, la compréhension du mouvement historique exige que l'on soit toujours attentif à l'inachèvement, à la diversité des systèmes et des interprétations nationales, sociales ou individuelles : «Les systèmes de pensée, comme les systèmes culturels, sociaux et politiques, sont des structures mobiles, dont le fonctionnement n'est jamais parfait, parce que l'intégration des éléments n'est jamais complète » (p. 68).

On peut appliquer ce principe à l'historiographie des années 1960-1980. Comme le montre bien Claude Blanckaert dans sa très riche introduction, J. Roger reconnaissait lucidement qu'à la suite du structuralisme « le rationalisme moderne a rendu historiens et philosophes plus attentifs à une pensée qui structure le réel en soulignant les discontinuités » (p. 22). Mais lui-même se refusait à une telle attitude qui mutilait la complexité et le mouvement de l'histoire réelle. Un chercheur actuel, tentant l'analyse de l'historiographie de ces années, se tromperait donc en caractérisant l'« épisteme » des historiens par une posture unique pour laquelle les « études de structures prennent visiblement le pas sur les études de genèse » (p. 227). En effet, l'œuvre de J. Roger l'obligerait à reconnaître la présence à cette époque d'une méthode spécialement attentive aux phénomènes de genèse, donnant une place centrale aux auteurs, ne refusant ni analyse biographique, ni considérations psychologiques. Et ce serait bel et bien cette richesse présente alors qui lui servirait à expliquer les développements de l'histoire des sciences dans les années qui suivirent.

Placée au cœur de l'histoire générale, l'histoire des sciences doit donc mobiliser pour ses explications à la fois la complexité sociale et institutionnelle, les controverses philosophiques et politiques, les rencontres entre traditions culturelles, les images qui structurent les croyances partagées et, finalement, laisser place à la psychologie des auteurs, à leurs styles et à leur imagination. Chaque moment de l'histoire doit contenir en lui-même les moyens et les règles de ses transformations. C'est ce même questionnement sur la causalité des phénomènes historiques qui explique la fascination de J. Roger pour les théories de l'évolution biologique, entendue comme histoire des formes vivantes : « [...] le problème du transformisme est de découvrir dans la nature actuelle les moyens d'une histoire » (p. 235). 
Pour comprendre l'histoire, les analyses des conditions de la réception ou du succès des théories et pratiques ne peuvent suffire, « la succession des théories, si intéressante qu'elle soit, simplifie à tort la complexité du processus et parler de "changement de paradigme" n'est pas d'un grand secours » (p. 211). Ce serait une forme de darwinisme qui expliquerait l'histoire par la sélection sans rendre compte de la causalité des variations. L'historien doit plutôt aussi tenter de saisir les conditions qui ont conduit à la construction des nouvelles théories. Cependant, montrer que la création théorique est dépendante des idéologies, des institutions et de toutes les composantes de la subjectivité ne doit pas conduire à une réduction sceptique du savoir scientifique aux conditions sociales de sa genèse.

Dans une certaine mesure, J. Roger admettait le principe de symétrie chère à l'actuelle sociologie des sciences : «Le problème de la vérité scientifique peut être l'affaire du philosophe ou du savant; il n'est pas, en soi, l'affaire de l'historien. L'histoire des sciences ne peut prétendre à aucune valeur normative » (p. 54). En effet : «La volonté de juger empêche souvent de comprendre » (p. 54). Cependant, contre le réductionnisme sociologique, il affirmait une spécificité du savoir scientifique : « [...] l'histoire même oblige à reconnaître une différence entre ce qui dure et ce qui ne dure pas dans des sociétés qui changent» (p. 58). La spécificité de la démarche intellectuelle scientifique tient à la pérennité de ses résultats, leur adaptabilité à des contextes sociaux ou idéologiques opposés. Ainsi, l'histoire des sciences contient une forme de critère de validité des connaissances scientifique, critère relatif et provisoire, consistant en la réappropriation de ces connaissances par d'autres auteurs, au service d'intérêts divers ou contradictoires. Sur une abondance d'exemples, J. Roger conjugue donc l'analyse de la construction d'une théorie dans un contexte socio-idéologique donné et la démonstration de sa relative indépendance vis-à-vis des causes de sa construction : «[...] la mécanique céleste newtonienne a largement survécu aux conditions politico-religieuses de sa naissance et de son succès initial [...]» (p. 58).

Un même idéal devrait guider la production des connaissances historiques. L'historien sait que ses choix et moyens sont conditionnés par le présent. "Mais s'il cherche vraiment à comprendre pour comprendre, il atteindra normalement à ce que l'on peut appeler l'objectivité historique. Ce qui se marque à ceci que les faits et les documents qu'il aura mis au jour ou les analyses qu'il aura produites pourront être utilisés par d'autres et peut-être pour d'autres interprétations » (p. 56). Une telle exigence entraînait celle de la lecture attentive des autres chercheurs, la mise en place d'un système de références systématique et le développement de descriptions complètes laissant toujours place à une complexité réelle qui dépasse l'analyse présente. C'est là ce qui explique la pérennité des travaux de J. Roger et justifie tout l'intérêt de ce recueil de textes. 
Franck Salaün, L'Ordre des moeurs. Essai sur la place du matérialisme dans la société française du xvıI siècle (1734-1784). Paris, Kimé, 1996. 14,5×21, 368 p. (« Philosophie-épistémologie »).

Franck Salaün se livre à une intéressante recherche sur la place du matérialisme dans la société française, entre la publication des Lettres philosophiques de Voltaire (1734) et la mort de Diderot (1784). Au début du siècle, le phénomène demeure rare et relativement discret. Le terme «matérialiste » entre tardivement dans la langue française : il est attesté en anglais dès 1668, mais n'apparaît en français qu'en 1702, dans la polémique entre Bayle et Leibniz. Mais pendant longtemps encore règne l'amalgame simplificateur : matérialisme, athéisme, déisme, sont souvent confondus dans l'anathème et désignent un adversaire protéiforme. L'accusation vengeresse est tour à tour portée contre Épicure et Lucrèce, mais aussi contre Spinoza, Bayle, Hobbes, Locke, Toland... et la liste serait sans fin.

Salaïn voit un tournant décisif dans l'histoire du matérialisme et de sa perception, avec la parution en 1745 de l'Histoire naturelle de l'âme de La Mettrie. Celui-ci défend ouvertement le matérialisme et la philosophie, comme si les deux notions étaient consubstantielles, et les oppose au fanatisme religieux, pour conclure: "Cent traités du matérialisme sont donc beaucoup moins à craindre qu'un janséniste impitoyable, ou qu'un pontife ambitieux. » Dans la seconde partie du siècle, autour de 1760 , le matérialisme est devenu un danger pressant, grave, qui mobilise contre lui les meilleures plumes de l'apologétique. L'avocat général Joly de Fleury stigmatise le complot «d'une société formée pour soutenir le matérialisme, pour détruire la religion, pour inspirer l'indépendance, et nourrir la corruption des mœurs ». $\mathrm{Au}$ milieu de nombreux autres textes dénonciateurs, l'abbé Gauchat affirme, en 1763, que le non-chrétien passe inévitablement au matérialisme et à l'athéisme : pas de milieu, pas de droit à l'indifférence. Aux alentours de 1780, la question du matérialisme semble cependant avoir perdu de son actualité polémique.

Salaün relie cette chronologie fine et bien argumentée au développement général des Lumières et notamment à la sécularisation du temps. Il se livre à une critique du célèbre texte de M. Foucault sur l'article, non moins fameux, de Kant publié en 1784 , en réponse à la question lancée par le périodique Berlinische Monatschrift «Qu'est-ce que les Lumières? ». Il conclut : "La sécularisation du temps, que de nombreux faits confirment, est à la fois l'une des formes de la matérialisation du réel et l'une des conditions des autres évolutions. Dans la France du xviII siècle, l'attention au présent accroît le sens du possible» (p. 98). Pour l'auteur, le xviII ${ }^{e}$ siècle est celui des mours. Le thème de leur corruption n'est pas nouveau et se retrouve dans la prédication et la pastorale. Mais avec Condillac, les mœurs «sont susceptibles d'être rapportées aux normes sociales ", elles sont conditionnées par l'histoire (p. 114), et pour d'Holbach « les mœurs doivent correspondre à la civilité véritable dont il trouve le modèle chez les bourgeois éclairés de la République des lettres. On peut, c'est entendu, lui reprocher de ne donner ainsi qu'un reflet des intérêts de sa classe, d'en être une sorte de porte-parole, Marx et Engels l'ont fait dans l'Idéologie allemande, mais il ne faut pas perdre de vue le contenu de ce modèle, qui, pour l'époque, est progressiste, si ce n'est révolutionnaire, et fait l'objet d'une réelle élaboration» (p. 214); notre baron n'est certes pas un révolutionnaire. S'il 
veut changer les mœurs, pour mieux changer la société, il tient à conserver les acquis de la civilisation, et à ne pas donner au peuple une liberté «trop étendue » qui risquerait de dégénérer «en licence». Salaün constate non sans regret : «Il semble que passé un certain seuil, d'Holbach, en tant qu'individu, résiste à sa propre philosophie, aux potentialités qu'elle porte, comme si ses mœurs étaient en retard sur son analyse matérialiste des mœurs » (p. 216).

Cet essai proclame sa volonté de lier recherche historique et réflexion philosophique : ce second point est parfaitement réussi, le premier laisse trop souvent insatisfait. Le thème de la « sécularisation » notamment mériterait une analyse précise. Bien avant le siècle des Lumières, J. Le Goff a perçu un temps des marchands, les Réformes marquent également un temps fort de cette sécularisation... La bibliographie fait appel aux meilleurs historiens, mais le lecteur a souvent l'impression qu'ils sont convoqués comme témoins, voire comme alibis, dans une problématique, très respectable, qui demeure celle de l'histoire des idées.

Monique CoTtret

« Kant : philosophie de l'histoire », Revue germanique internationale, 6, 1996.

Précisons d'emblée que le lecteur trouvera, en fin de volume, une utile bibliographie consacrée à la question de la philosophie de l'histoire. Norbert Waszek, dans sa présentation du volume, définit l'enjeu central de cette publication : montrer, suite au bicentenaire du traité Vers la paix perpétuelle de 1795, l'actualité de la philosophie kantienne de l'histoire et souligner son intérêt philosophique majeur. Le volume se distribue en trois sections traitant successivement de la généalogie et des sources de l'histoire kantienne, des concepts majeurs de cette histoire (le droit, le contrat, la téléologie, les indices et la question de la pratique) et de sa postérité néokantienne et hégélienne.

Reinhard Brandt n'hésite pas à faire de l'anthropologie kantienne « le lieu de naissance de la philosophie kantienne de l'histoire» (p. 22). Ce que montrent les cours d'anthropologie de Kant, bientôt réunis dans le tome XXV de l'édition de l'académie de Berlin, c'est une grande proximité de la réflexion kantienne avec la tradition des philosophes populaires (Popularphilosophen), tels Christian Garve et J.G.H. Feder, et leur commun intérêt pour une philosophie de la vie quotidienne. Ce qui est en jeu, en effet, dans une telle anthropologie, c'est de rechercher, à l'opposé de l'anthropologie physiologique et médicale d'un Ernst Platner, les sources de l'activité pratique de l'homme. On trouvera, dans cette analyse de la dimension pragmatique de la vie humaine dans son commerce prudent avec le monde, la matrice de la réflexion kantienne sur l'histoire.

Brigitte Geonget étudie minutieusement la généalogie du concept d'insociable sociabilité qu'elle rapporte à l'influence conjointe de Hobbes et de Rousseau. Elle montre que le concept de nature fonctionne à deux niveaux, celui métaphysique d'un agent téléologique et celui psychologique d'un lieu d'expression de nos affects passionnels. L'insociable sociabilité constitue le modèle paradoxal d'une socialité 
humaine ambivalente, où le lien intersubjectif se forme à partir d'une relation première de conflit et d'hostilité. Tel est le sens de ce fameux oxymore.

La philosophie kantienne de l'histoire s'oppose radicalement aux Ideen de Herder. Ce que montre Pierre Pénisson, c'est que la confrontation est d'abord celle de deux conceptions de l'écriture philosophique. L'une, poétique, celle de Herder, court sans cesse le risque de l'imprécision imaginative, tandis que l'autre, dessinée en creux, celle bien sûr de Kant, reste attachée à la rigueur et à l'exactitude des concepts. Plus fondamentalement, Kant souligne l'effroi de la raison confrontée à la vacuité des idées herderiennes. Ce qui lui paraît condamnable, c'est à la fois le goût de Herder pour la diversité sensible (telle celle des époques historiques) contre l'unité du concept et son penchant pour une histoire naturelle de l'homme, qui est à l'opposé d'une philosophie de la liberté et de ce qui en constitue le corollaire, la définition d'un statut philosophique de l'avenir.

Reste à révaluer l'influence écossaise sur Kant. Norbert Waszek montre que le thème kantien d'une liberté politique s'engendrant à partir du développement du commerce et des industries ne peut se comprendre que si l'on fait référence à la réflexion de Hume concernant le luxe. Ce que Kant fait apparaître ici, en écho aux philosophes écossais, c'est l'équivalent d'une ruse de la raison historique : le despotisme ne peut porter atteinte à la liberté civile et donc au développement économique du pays, sans contradictoirement l'affaiblir par rapport aux états extérieurs.

Le deuxième volet de ce tryptique concerne les grands thèmes de la réflexion kantienne sur l'histoire. Dans une intervention d'une grande densité conceptuelle, Bernard Bourgeois reconstruit les différents moments de l'analyse kantienne du droit. Ce qu'il faut élucider ici, c'est l'équation de l'histoire et du droit. Celle-ci ne peut se lire qu'en un seul sens : si l'histoire est droit, le droit n'est pas histoire. Le kantisme n'est pas un historicisme, car la raison pratique surplombe verticalement l'histoire, au lieu de rendre possible une production horizontale du droit dans l'histoire comme chez Hegel. On trouvera là ce qui distingue radicalement l'entendement juridique kantien de la ruse de la raison hégélienne. Reste que l'équation doit se lire dans le premier sens évoqué. Le moment juridico-politique de la civilisation rend possible le développement culturel de l'habileté et celui, éthique, de la moralité. L'origine, le cours et la fin de l'histoire ne peuvent se penser que par rapport à la républicanisation de l'État.

La théorie du contrat occupe une place centrale dans l'économie de la pensée historique de Kant. Karlfriedrich Herb fait l'inventaire de toutes les transformations que Kant impose à cette notion. Kant change de terrain par rapport à Rousseau. La notion devient une pure idéalité juridique, toute dimension historique étant écartée. Le rapport du droit public et du droit privé est inversé par rapport à Rousseau. Il ne s'agit plus de produire une individualité sociale et politique. De même, la question de la représentation politique oppose les deux philosophes. Le contractualisme ouvre pour l'un à un optimisme confiant, pour l'autre à un grand pessimisme quant à l'utilité d'une grande science de la politique.

Jean-Michel Muglioni souligne l'importance du principe téléologique dans la construction de la pensée kantienne de l'histoire. Qu'est-ce que la réflexion téléologique sinon l'entreprise qui fait parler l'histoire dans le sens de l'intérêt de la raison? De cette réflexion, on attendra des signes et non des preuves, une espérance et non une science. La réflexion de cette finalité pratique commande celle de la finalité 
naturelle. Comme le soulignait Éric Weil, le cosmos ne possède un sens que pour l'homme qui y réalise sa liberté. La téléologie, c'est la raison pratique comme fil conducteur et principe d'orientation.

L'histoire, comme le montre Michèle Crampe-Casnabet, est d'abord celle de la raison, d'une enfance dogmatique à l'âge critique en passant par l'adolescence anarchique. Le traité sur le conflit des facultés systématise cette conception progressiste de l'histoire : il faut récuser à la fois la conception terroriste (l'histoire comme régression) et la position abdéritaine (l'oscillation pendulaire du bon et du mauvais), au profit d'une conception prophétique d'une histoire allant vers le mieux. Cette méditation de l'histoire prend sens à partir d'une lecture des indices qu'offre le mouvement de l'histoire.

Heiner F. Klemme replace le débat sur la théorie et la pratique dans la constellation historique qui lui donne sens (la philosophie populaire d'un Thomasius). Ce que Kant veut montrer contre ses détracteurs, c'est que de la théorie morale à la pratique, la conséquence est bonne. Il n'y a pas, en effet, de solution de continuité du « je dois » au « je peux ». C'est dans ce cadre conceptuel que prend sens la référence au droit de résistance ainsi qu'au droit international dont Kant montre pour quelles raisons il est tout autre chose qu'une chimère. Pour finir l'auteur donne l'exemple suggestif de l'enseignement comme passage à la pratique morale, tout en montrant la constitution d'une méthodologie pratique qui vise à faire naître l'intérêt pour la raison pratique. De sorte qu'il faut dépasser, comme le fait la philosophie populaire, l'opposition de l'école et de la vie.

Helmut Holzhey étudie la postérité de la réflexion kantienne dans l'œuvre de Hermann Cohen. La thématique centrale de l'école de Marburg est ici de savoir comment le «devoir-être» peut trouver son «être». Le fait recherché sera la pure science du droit politique et non l'histoire. Si le droit est bien le véhicule de l'histoire, il faut s'interdire toute spéculation religieuse sur la finalité de l'histoire et occuper le poste qui est le nôtre dans l'histoire. Ce qui constitue sans doute le meilleur moyen d'éviter toute dérive totalitaire.

Jacques D'Hondt restitue tout le caractère explosif de l'opuscule de Kant sur l'histoire universelle. Kant commence par écarter toute investigation métaphysique de la question de la volonté pour mettre en évidence l'enchaînement causal des phénomènes historiques. Puis il souligne la nécessité d'une socialisation et d'une association cosmopolitique de l'homme. Cette histoire du genre humain se fera, dans une véritable ruse de la raison, en utilisant les mauvais instincts de l'homme.

Ce numéro de la Revue germanique internationale, fort riche, a le mérite de réévaluer certaines sources de l'investigation kantienne de l'histoire (la philosophie populaire et la pensée écossaise) et de proposer une étude conceptuelle précise des grands concepts kantiens de l'histoire. 
Chantal Grell, Le Dix-huitième Siècle et l'Antiquité en France, 1680-1789. I et II. Oxford, Voltaire Foundation, 1995. 16,5 × 24, xxIII-1335 p., bibliogr., index («Studies on Voltaire and the eighteenth century », 330-331).

Le grand travail de Chantal Grell reprend et amplifie une recherche sur la culture historique des Lumières françaises déjà présentée dans la Revue de synthèse (2-3, avr.-sept. 1995 , p. 487-488). Avec le présent volume, il s'agit du rapport de la culture française du $\mathrm{XvIII}^{\mathrm{e}}$ à l'Antiquité dans la totalité de ses aspects. La première partie examine les cadres et les sources de la connaissance (collèges, académies, textes et monuments). La deuxième partie se demande comment fonctionne le modèle antique : dans quelle mesure est-il rejeté par les « Modernes » et pourquoi ? Quels sont les remaniements, voire les distorsions que subit le modèle quand il est réutilisé par la pensée politique ou l'œuvre d'art (architecture, sculpture, peinture)? À partir de quels centres se diffusent les modèles antiques remaniés? La troisième partie dégage les enjeux politiques, religieux et éthiques des débats menés autour de l'Antiquité.

La première conclusion qui se dégage, à la lecture de cette enquête exhaustive, est le peu de science qu'ont fourni les milliers de pages que l'auteur a lues pour nous (1500 titres environ). Certes, il faut rendre hommage aux doctes et silencieux travaux de l'Académie des inscriptions et belles lettres; reconnaître le flair méthodologique de Louis de Beaufort, de Nicolas Fréret. Mais la science historique française, appliquée à l'Antiquité, n'atteint pas, sauf à Strasbourg, le sérieux de l'Altertumwissenschaft. D'une part, la méthode critique lumineusement mise au point par Mabillon et ses émules ne peut s'appliquer à l'Antiquité, faute de documents suffisants ou suffisamment fiables. D'autre part, l'histoire en France s'offre comme une discipline éclatée : se la partagent les Belles-Lettres, l'érudition et la philosophie. Dans la pondération des influences, cette dernière l'emporte. L'histoire en France possède des ambitions philosophiques à cause de l'importance des enjeux qu'elle enveloppe. Le camp des philosophes travaille à l'arracher à la domination de l'apologétique et $\mathrm{C}$. Grell montre bien comment cette préoccupation renouvelle la conception de l'histoire des religions. La Bible cesse d'être le grand livre du savoir, ne serait-ce que par la fausseté de sa chronologie. Mais cette sécularisation de l'histoire n'est-elle pas un faux-semblant, un simple transfert de sacralité? C. Grell incline à le croire, en appuyant de toute son érudition une proposition de Starobinski : la recherche d'une origine immaculée, du peuple « instituteur de l'humanité » garde une saveur théologique.

À côté de la religion, la politique. Le recours au modèle antique s'opère de manière contradictoire selon les camps en présence. Les partisans de la monarchie absolue relèvent toutes les institutions ou les événements de l'Antiquité qui justifient la volonté royale de mettre au pas la noblesse ou les Parlements. Les opposants, attachés aux vestiges de la société féodale, insistent sur le despotisme des sociétés antiques et regardent les conquêtes barbares comme une «libération », afin de justifier les coutumes franques et les privilèges seigneuriaux. L'acharnement de ces discussions finira par lasser l'opinion, comme le montre, en l'an III, une leçon de Volney prononcée à l'École normale supérieure. Dans sa conclusion, C. Grell cite longuement ce texte hautement significatif. 
Les deux branches de l'enquête finissent ainsi par se rejoindre. D'un côté, la science vraie de l'Antiquité est assez pauvre dans la France des Lumières. De l'autre, les Français, habitués de longue date à assimiler dans les collèges l'héritage humaniste, vivent au milieu des modèles antiques. La circulation des artistes entre Paris et Rome et la permanence dans l'art des sujets et des styles antiques renforcent cette imprégnation de leur sensibilité. L'Antiquité leur est si présente qu'elle a de la peine à acquérir le détachement nécessaire pour devenir objet d'histoire. Il faudra, pour que naisse en France une science de l'Antiquité, non seulement les développements de la philologie et les conquêtes archéologiques du $\mathrm{XIX}^{\mathrm{e}}$ siècle, mais aussi le changement du regard. L'Antiquité apparaîtra dépouillée de ses revêtements chrétiens et dénuée de recettes politiques pour le présent, puisque, décidément, la liberté des Modernes se distingue de celle des Anciens. Si l'on ajoute à ces facteurs la prise de conscience du progrès de l'esprit humain, l'attention à l'avenir provoquée par le développement des sciences, l'on sera amené à conclure, de la naissance de la modernité et du rejet de la tradition qui la définit, à la possibilité de construire le passé comme objet de science, au lieu de subir sa domination culturelle.

L'enquête de C. Grell se recommande pour son sérieux et son exhaustivité. Elle s'étend à tous les domaines de la culture et réserve une large part à l'histoire de l'art, bien incorporée à l'ensemble du sujet. Certes, l'antiquité gréco-romaine tient la première place dans le livre, comme il se doit, mais les civilisations du Moyen et de l'Extrême-Orient ne sont pas en dehors de l'enquête, puisque les Lumières françaises s'y intéressèrent de plus en plus. La qualité de l'érudition de l'auteur est mise en valeur par ses notes, précises et complètes, ainsi que par ses abondantes bibliographies et les tableaux offrant des indications quantitatives sur la répartition, à diverses périodes, de la production imprimée relative à l'Antiquité. C. Grell a pris un soin particulier à noter l'apparition des termes nouveaux, significatifs pour son sujet, et à décrire la généalogie des concepts ainsi désignés (ainsi les termes désignant les périodes historiques : Antiquité, Moyen Âge, Renaissance; ou d'autres comme : mythe, révolution...). Les analyses de contenu sont précises et nuancées, parfois un peu longues, mais ce léger défaut me paraît, à tout prendre, moins imputable à l'auteur qu'aux normes quantitatives qui définissaient, dans un Urzeit, la longueur souhaitée pour l'ancienne thèse de doctorat d'État. C. Grell s'est attachée de manière soutenue à mettre en contexte les contenus des œuvres étudiées et à les rattacher à la fois à des milieux socioculturels et à la conjoncture politique. Elle donne ainsi une claire vision des oppositions qui structurent le champ social de la France du XVIII siècle : Versailles (la cour) vs. Paris (la ville); monarchie vs. parlements/ noblesse; jésuites vs. jansénistes; Anciens vs. Modernes; romanistes vs. germanistes. Entre ces différents clivages de la société française, s'opèrent des glissements et des superpositions provisoires, en fonction des stratégies du moment. Tous ces mouvements sont scrupuleusement observés et, au total, ce livre apporte un enrichissement indispensable à notre connaissance du xviII ${ }^{e}$ siècle français.

François LAPLANCHE 
Pascale Hummel, Humanités normaliennes. L'enseignement classique et l'érudition philologique dans l'École normale supérieure du XIX' siècle. Paris, Les Belles Lettres, 1995. $16 \times 24,297$ p., bibliogr., index («Études anciennes. Série grecque », 123).

Mettant à profit sa compétence de philologue et sa connaissance des sources de l'histoire de l'École normale supérieure ${ }^{1}$, Pascale Hummel retrace dans ce travail ce que fut, dans cette institution, l'enseignement des humanités au long du $\mathrm{XIX}^{\mathrm{e}}$ siècle.

L'ouvrage s'ouvre naturellement sur cette étape préliminaire qu'est le concours d'entrée qui, dans le prolongement du secondaire, fait la part belle au latin, « le véritable pilier du système éducatif français »; le grec occupe une place seconde. Cette hiérarchie ressort du nombre des épreuves : à l'écrit, une seule pour le grec (version jusqu'en 1842, puis thème), contre trois pour le latin jusqu'en 1885 (discours, version, vers). Elle se dégage aussi de la nature même de ces épreuves et des performances des candidats : alors que le niveau en grec est bien inférieur, la version, puis le thème servent à vérifier une compétence linguistique; il en va différemment pour le latin qui, supposé parfaitement maîtrisé (et n'oublions pas la différence de traitement entre les deux langues tout au long de la scolarité au lycée), est « une langue véhiculaire à laquelle il est demandé d'exprimer des idées et surtout de faire preuve d'une dextérité rhétorique ».

Trois chapitres portent ensuite sur l'enseignement des humanités à l'École normale. Le premier examine les principes qui le guidèrent et les méthodes qui furent mises en œuvre : la « conférence », l'enseignement mutuel, l'enseignement individuel, l'enseignement simultané, autant de pratiques qui répondent au souci de concilier une formation savante individuelle et de préparer les élèves à leur future tâche de professeur. L'auteur examine ensuite les contenus de cet enseignement et les exercices auxquels se livraient les élèves pendant les deux, puis trois années qu'ils passaient à l'École. Enfin, elle s'arrête sur les maîtres qui, pendant un siècle, distribuèrent cet enseignement : il s'ensuit une longue "galerie de portraits " où se succèdent hellénistes, latinistes, grammairiens et suppléants, autant de portraits qui valent surtout par les témoignages que leurs anciens élèves ont laissés. Les deux principales conclusions qui se dégagent de ce chapitre de «prosopographie » sont une tendance croissante à «l'autorecrutement ou recrutement interne » et une certaine diversité quant aux talents, ce que P. Hummel énonce ainsi : «Les quelques noms qui retiennent l'attention dans le cortège des maîtres qui professèrent à l'École normale au $\mathrm{XIX}^{\mathrm{e}}$ siècle sont comme les derniers témoins des humanités vivantes incarnés dans des personnalités parfois attachantes. »

Le chapitre $v$ replace les humanités normaliennes dans le cadre des études classiques en France. La comparaison avec l'instruction reçue au lycée est riche d'enseignements, en particulier quant au statut des deux langues classiques : le grec fut mis sur un pied d'égalité avec le latin et les élèves, peut-être lassés des longues années et

1. Pascale Hummel, Regards sur les études classiques au xix siècle. Catalogue du fonds Morante de l'École normale supérieure, Paris, Presses de l'École normale supérieure, 1990. ID., Anne Lejeune, David Peyceré, Pour une histoire de l'École normale supérieure: sources d'archives (1794-1993), Paris, Archives nationales/Presses de l'École normale supérieure, 1996. 
des nombreuses heures de latin du lycée, embrassèrent le grec avec enthousiasme et avec un certain succès. Les exercices et les méthodes de travail différaient aussi, laissant plus de place à l'oral (il s'agit de former de futurs professeurs) et à l'initiative individuelle, à la recherche : ici, les élèves avaient le privilège de disposer de plusieurs bibliothèques et dépôts de livres particulièrement riches quant au fonds sur l'Antiquité classique. Rien d'étonnant donc à ce que l'on trouvât les normaliens en nombre, comme étudiants, auditeurs, ou professeurs, à la Sorbonne ou à l'École pratique des hautes études, qu'ils aient contribué massivement aux publications pédagogiques en matière d'études classiques, parfois même avec des innovations promises à un bel avenir, telles les traductions juxtalinéaires lancées en 1845 par Louis Hachette, un ancien normalien.

Le sixième et dernier chapitre replace l'enseignement qui fut distribué à l'École normale, les réformes et les aménagements qu'il connut, dans le cadre d'une confrontation, particulièrement aiguë dans le dernier tiers du siècle, avec le modèle allemand. Alors qu'en France l'approche de l'Antiquité classique était plus esthétique que savante, plus morale que scientifique, alors que l'enseignement privilégiait des qualités littéraires et rhétoriques qui avaient leur dénominateur commun dans le goût, l'Allemagne de l'Altertumwissenschaft exerça au long du $\mathrm{XIX}^{\mathrm{e}}$ siècle une séduction de plus en plus forte; des voyages et des séjours outre-Rhin, la lecture d'ouvrages allemands (et la bibliothèque de l'École normale supérieure était dans ce secteur plus riche que la Bibliothèque nationale), la traduction de tels ouvrages, des correspondances avec des savants allemands, la présence de maîtres allemands à l'École familiarisèrent les normaliens avec une approche érudite et amenèrent à une réévaluation de la grammaire et à l'introduction de nouveaux enseignements de paléographie, de philologie, d'histoire ancienne. Cet intérêt pour la science allemande ne fut pas unanime et l'on rechercha une voie moyenne qui serait « le mélange d'une science pure et du brio mondain ».

Cet ouvrage que nous avons présenté dans ses grandes articulations repose sur une véritable somme d'érudition qui amène d'ailleurs l'auteur à fournir des informations aussi nombreuses que précises sur tous les points qu'elle traite. Ainsi, pour le concours, elle retrace l'historique de sa mise en place, de son organisation; il en va de même pour la «conférence ", pour le statut du corps professoral, pour la constitution des bibliothèques, etc. De sorte que l'ouvrage donne beaucoup plus que son titre ne promet. Il ne laisse pas moins le lecteur parfois sur sa faim. Ainsi, alors que l'auteur nous dit disposer des feuilles de notes (c'est-à-dire d'appréciations portées sur les élèves au cours de leur scolarité), on eût aimé une étude détaillée sur le niveau des normaliens en latin et en grec. De même, la « question du latin » est trop rapidement évoquée; on aurait souhaité en savoir plus sur l'écho qu'eut à l'École normale le débat autour des « humanités modernes »; encore n'aurait-il pas été sans intérêt d'analyser les arguments qui s'échangèrent lors de la suppression des vers latins, ne serait-ce que dans le cadre plus général du débat de fond entre l'humanisme à la française et la science allemande. Enfin, l'auteur n'échappe pas aux redites et, par un goût prononcé pour la nuance, en arrive parfois à manier un style excessivement lourd ${ }^{2}$. En conclusion, il eut été à souhaiter que cet ouvrage, fondé

2. Pour un exemple : «Alors que l'École normale fut créée par des révolutionnaires plus soucieux de rupture que de continuité, la procédure de recrutement dotée par Napoléon des 
sur une documentation et une bibliographie impeccables, fît un peu plus de place au brio et que l'érudition le cédât à l'histoire.

Françoise WAQUET

Victor Hugo et l'Europe de la pensée. Colloque de Thionville-Vianden, 8, 9 et 10 oct. 1993, textes réunis et présentés par Françoise Chenet-Faugeras. Paris, Nizet, $1995.16 \times 24,247 \mathrm{p}$.

Projet séduisant et généreux, le colloque qui s'est tenu en octobre 1993 à la fois à Thionville et à Vianden, deux hauts lieux hugoliens et européens, s'intitulait «Victor Hugo, les Intellectuels et l'Europe », ce qui ouvrait bien des directions. Les actes du colloque ont reçu un autre titre, Victor Hugo et l'Europe de la pensée, et ont été construits selon trois parties : « Pré-histoire », « Victor Hugo et l'Europe », "L'Idée européenne en marche ». Le lecteur a bien une impression de disparate (c'est un peu la loi du genre) mais il trouvera au fil des pages de quoi nourrir sa curiosité et sa réflexion.

Françoise Chenet-Faugeras, qui a organisé le colloque et préparé ces actes, éclaire le projet en revendiquant une approche "génétique et plus philosophique que littéraire» de l'idée d'Europe; dans sa propre communication («Victor Hugo et l'Europe : esprit du lieu et lieu des esprits »), elle définit le colloque comme une manière de faire l'Europe en la disant, une sorte d'épiphanie à la fin du $\mathrm{xx}^{\mathrm{e}}$ siècle de l'idée que se faisaient de l'Europe les esprits du milieu du $\mathrm{XIX}^{e}$ siècle : un « concile des intelligences ». Certains articles répondent parfaitement à ce projet (et ils justifient largement l'existence du recueil) tout en ayant également le mérite de souligner les ambiguités d'une certaine idée de l'Europe et la conscience que Victor Hugo et d'autres « intellectuels » du XIX siècle avaient de ces ambiguittés.

L'ouvrage propose plusieurs monographies d'intérêt très inégal : on s'interroge par exemple sur la pertinence, par rapport au recueil, de la mise au point sur les saint-simoniennes mais on goûte la vivacité du croquis des chevaliers de fortune (ces aventuriers européens du $\mathrm{xvuH}^{\mathrm{e}}$ siècle) ainsi que la précision pittoresque des documents sur les séjours de Hugo au Luxembourg.

Plus substantielles sont les deux « ouvertures » qui, sous le signe de Platon et de Dante respectivement, posent d'emblée l'Europe non comme une entité géographique mais comme un principe spirituel dynamique, l'âme rayonnante du monde. On se laisse guider avec beaucoup d'intérêt par Jean-François Mattei, du mythe originel d'Eurôpè (la princesse asiate dont le nom même et le destin tracent la vocation

fondements juridiques et institutionnels d'un concours, pouvait difficilement épouser les desseins iconoclastes des plus ardents, si bien qu'elle se caractérisa d'emblée, et se caractérise encore mutatis mutandis, par une évidente continuité par rapport et à conformité avec l'enseignement secondaire, qui fait de cette École, comme de beaucoup de grandes écoles, des lieux parfois controversés pour leur conformisme » (p. 58). 
universelle de l'Europe) à sa transposition philosophique chez Platon et à l'influence de celui-ci sur les images hugoliennes de l'Europe (que l'auteur voit organisées selon deux axes, le premier d'expansion horizontale, le second de transcendance verticale). Maurice de Gandillac trace l'extraordinaire dynamique de l'Europe médiévale (en train de prendre une fière conscience d'elle-même), son cosmopolitisme et son universalisme fondés sur la raison et l'esprit critique et il en montre les échos dans les images de Dante.

Les conceptions européennes de Hugo sont abordées de manière intéressante soit à travers ses « actes et paroles » (Yves Gohin analyse la transfiguration rêvée de la France en une Europe-fédération de peuples par un processus de sublimation, analogue à celui par lequel le Peuple deviendra Humanité), soit à travers ses œuvres proprement littéraires (Priscilla Parkhurst-Ferguson cerne dans Quatrevingt-Treize, les jeux d'opposition entre Paris, la France et l'Europe et montre comment la langue hugolienne fait entrer le particularisme vendéen dans une « francité » dont la mobilité et la modernité ont vocation universelle, avec tous les germes impérialistes que cela implique). Franck Laurent, enfin, donne une approche très fine et très ferme de la position hugolienne des années 1840 (autour du Rhin principalement); il montre que l'assimilation entre Europe et civilisation est, en même temps et avec autant de force, affirmée et minée par Hugo, autant par sa vision de l'Orient que par sa conscience de tous ceux qu'exclut le discours de la Civilisation, en particulier les «barbares de l'intérieur».

Les thèses européennes de Novalis d'une part, de Maistre et Veuillot d'autre part, sont présentées sans toujours les mises en perspective que l'on pourrait attendre, mais elles rappellent utilement combien fut grande la nostalgie d'une Europe définie comme et par la chrétienté. Dans le même ordre d'idées, l'article sur Auguste Comte montre combien sa conception de l'occidentalité comme étape vers l'humanité implique le rapport le plus étroit qui soit entre Europe et spiritualité.

Ce recueil, donc, s'il célèbre l'Europe comme principe spirituel rayonnant, met fortement en soupçon une certaine idée de l'Europe au $\mathrm{XIX}^{\mathrm{e}}$ siècle mais aussi au $\mathrm{Xx}^{\mathrm{e}}$ (Jean Maurel dénonce avec véhémence la fermeture des intelligences européennes). L'ouvrage est traversé par les interrogations sur la «mission » de l'Europe et sur une éventuelle téléologie de son histoire, renvoyant constamment à la question de sa définition même et du lien de cette définition avec la notion de Civilisation, ce qui amène forcément la confrontation avec la Barbarie (la contribution de Pierre Michel, largement nourrie de Michelet, montre combien l' «Européen essentiel » intègre des éléments de barbarie et elle entre par là en écho avec les analyses sur les «barbares " de Hugo). Affleure aussi dans maint article l'articulation problématique entre le particulier et l'universel (dont Monique Castillo situe très bien les enjeux à travers une confrontation entre les thèses de Kant et celles de Herder sur l'opposition entre la civilisation et les cultures).

Ce qui reste posé, c'est le risque incessant de glissement de l'universalité à l'impérialisme. C'est une des gloires de Victor Hugo que d'avoir senti les pièges du rêve européen et du discours de la Civilisation - et ce, avant même qu'il ne soit devenu républicain. Le colloque, et ces actes, auront montré combien Hugo, de multiples manières, a intégré et problématisé les discours de son temps. C'est peut-être 
en ce sens (et parce qu'il est devenu anti-impérialiste) qu'il est vraiment européen et universel.

Agnès SPIQuel

Ernest Renan, L'Avenir de la science. Prés., chronol., bibliogr. par Annie Petrt. Paris, Flammarion, 1995. $11 \times 18,542$ p. («GF-Flammarion », 765).

Le centenaire de la mort de Renan a donné occasion à des rééditions d'œuvres significatives, comme les Études d'histoire religieuse (Paris, Gallimard, «Tel», 1992). Annie Petit, professeur à l'université de Montpellier, nous donne maintenant une édition de l'Avenir de la science, d'après l'édition de 1890, qu'elle a collationnée avec le manuscrit. Elle indique que ce livre, écrit entre mai 1848 et juillet 1849 , mais publié seulement en 1890 , constitue à la fois un programme et un testament. Renan, en effet, y a puisé souvent et l'inspiration de ce texte est reconnaissable dans maint article de la maturité de l'auteur.

Cette édition comporte une chronologie très précise et une bibliographie de Renan (p. 53-62). L'introduction (p. 7-45) tire de ce texte bouillonnant, juvénile, quelques affirmations décisives pour l'intelligence de la pensée renanienne. A. Petit précise comment Renan se distingue du positivisme : sa religion n'est pas celle de l'humanité, mais celle de la science. Ce rationalisme critique s'applique de préférence à l'histoire de l'esprit humain, nulle part mieux saisissable que dans le moment originaire des grandes religions de salut. Les religions sont, en effet, "l'expression la plus pure et la plus complète de la nature humaine » (p. 307) et, pour chaque cuvre de l'esprit humain, l'origine est à privilégier, en tant que moment synthétique et spontané de ce qui, au fil du temps, deviendra plus analytique et plus réfléchi. Chaque religion déroule sa propre histoire selon des lois soumises à un déterminisme semblable à celui des lois de la nature.

Toutefois, A. Petit note que Renan assouplit ce déterminisme en insistant sur le rôle de l'observation concrète, même minutieuse, dans l'élaboration de la science historique. Mêmes nuances s'il s'agit de discerner les sources de la philosophie renanienne de l'histoire. Elles s'avèrent nombreuses (Goethe et Herder, Cousin, Hegel, l'évolutionnisme scientifique), mais tout est repris dans un effort de synthèse personnelle.

Enfin, la complexité du personnage et de l'œuvre se manifeste dans l'engagement politique. Chez Renan, c'est le rationalisme scientifique qui engage la vision politique : la vraie révolution n'est pas à attendre de transformations sociales, mais d'une réforme intellectuelle et morale. D'où l'accusation d'élitisme portée parfois contre l'auteur de L'Avenir de la science. Au total, les observations d'A. Petit invitent à une lecture fine du programme de Renan, texte majeur du $\mathrm{xIX}^{\mathrm{e}}$ siècle. 
Paul-Laurent Assoun, Freud, la philosophie et les philosophes. Paris, Presses universitaires de France, 1995. 12,5 × 19, 400 p., index ("Quadrige », 180).

Cette nouvelle édition de Freud, la philosophie et les philosophes, vingt ans après la première, reprend le texte initial en lui ajoutant d'une part une longue préface, d'autre part une troisième partie qui clôt l'ouvrage.

Dans la préface, Paul-Laurent Assoun s'explique sur les raisons qui l'ont amené, vingt ans après, à ajouter cette troisième partie. Cette dernière, en réalité, développe et complète la deuxième partie, consacrée à Freud et les philosophes. Nous analyserons donc la préface avec la deuxième et la troisième partie de l'ouvrage.

La première partie, «Freud et la philosophie » est nettement distincte des deux suivantes et représente, selon nous, la partie la plus riche et la plus intéressante de l'ouvrage. Aussi allons-nous dans cette analyse lui accorder une place plus grande qu'aux deux suivantes.

Cette première partie traite du rapport, ou plutôt des rapports contradictoires que Freud a entretenus, tout au long de sa vie, avec la philosophie, d'abord en tant qu'homme, ensuite et surtout en tant que penseur, rapports donc aussi bien théoriques qu'affectifs. Or, si l'on en croit précisément les propos de Freud quant à la philosophie, les options théoriques sont toujours à interpréter comme symptôme psychologique, et par suite l'affectif et le théorique ne sont que les deux versants d'un seul et même élan. Cet ouvrage ne prend peut-être pas les dires de Freud avec suffisamment de recul pour pouvoir les retourner sur Freud lui-même. En effet, on a souvent l'impression d'assister à une sorte de recensement des différents passages où Freud évoque la philosophie sans que ces propos soient euxmêmes interrogés, ne serait-ce qu'avec la grille de lecture que Freud applique aux écrits théoriques. Freud est lu comme jamais il n'aurait lu lui-même aucun théoricien. Bien sûr - et c'est ce que souligne Assoun - Freud ne se considérait pas comme un pur théoricien et ne considérait pas sa pensée comme un système. Or, c'est justement cela qu'il aurait peut-être fallu interroger : la théorie psychanalytique n'est-elle pas un système, qui, en tant que tel, pèche par les travers énoncés par Freud lui-même?

Ce livre a pourtant le mérite d'exposer de manière extrêmement claire les critiques que Freud assigne à la philosophie. Mais c'est cette clarté même qui nous pousse à considérer la pensée freudienne à la lumière de ces critiques et nous fait regretter que ce retournement ne soit pas développé.

Freud reproche à la philosophie deux aspects essentiels : son conscientialisme et le fait qu'elle se donne la plupart du temps comme une Weltanschauung, comme une vision du monde. Pourtant, malgré la force de ces critiques et le caractère rédhibitoire qu'elles ont pour lui, ce dernier demeure dans un rapport ambivalent à la philosophie, un rapport d'amour-rejet, et c'est ce que l'enquête biographique menée par Assoun dans l'introduction révèle. On est alors frappé de l'assiduité de cet étudiant en médecine pour les cours de philosophie. C'est quelque cinquante années après cet engouement premier pour la philosophie que Freud écrit à son 
propos dans une lettre adressée à Max Eitigon : «Vous n'imaginez pas combien me sont étrangères toutes ces cogitations philosophiques. La seule satisfaction que j'en retire est de savoir que je ne participe pas à ce lamentable gâchis de pouvoirs intellectuels » (p. 7).

Il faut donc revenir aux principales critiques que Freud énonce afin de se demander d'une part dans quelle mesure elles justifient la violence des propos précédents, d'autre part dans quelle mesure ces critiques ne s'appliquent pas finalement à Freud lui-même. Freud serait-il d'autant plus virulent qu'il se sentirait lui-même menacé, dans l'élaboration de sa pensée, par ces travers?

La première de ces critiques, celle du conscientialisme, ne peut évidemment pas se retourner contre Freud lui-même, mais paradoxalement paraît moins virulente que les suivantes. Il s'agit $d u$ point de divergence théorique entre la philosophie et la psychanalyse: la philosophie ne prend pas au sérieux l'hypothèse de l'inconscient. Même quand elle parle de pensées inconscientes, elle n'en prend pas la mesure. Elle reste sourde à l'inconscient, assimilant, en réalité, pensée et conscience. L'inconscient se réduit alors à ce qui est susceptible de conscience, à ce qui n'est pas actuellement dans le champ de la conscience mais peut être à tout moment réactualisé. L'inconscient de la philosophie n'est autre qu'un conscient virtuel. La spécificité de l'inconscient reste donc impensée. Or, si l'inconscient est resté ainsi impensé chez les philosophes, c'est qu'ils demeurent dans une interrogation stérile quant à sa nature sans se confronter au fonctionnement concret des processus inconscients. Ils ont laissé échapper tout l'aspect expérimental, c'est-àdire observable des phénomènes inconscients. Ainsi, c'est la méthode même de la philosophie (son abstraction et son absence d'observation concrète) qui la rend aveugle à la réalité spécifique de l'inconscient. La philosophie occulte ainsi l'objèt privilégié de la psychanalyse.

La seconde critique, qui consiste à souligner la propension de la philosophie à construire des «visions du monde», peut aisément se retourner contre la pensée de Freud lui-même. Si cette critique est si virulente, c'est qu'elle menace la théorie psychanalytique et que Freud le sait.

Freud oppose la Weltanschauung (vision ou conception du monde) et la Naturwissenschaft (science de la nature), en affirmant la proximité de méthode de cette dernière avec celle de la psychanalyse. La psychanalyse est avant tout une science empirique. La philosophie, en tant que vision du monde, construit des systèmes fermés prétendant expliquer la réalité tout entière à partir d'un principe déterminé a priori. Un tel système est sans doute cohérent mais perd en réalité ce qu'il gagne en cohérence; il est souvent totalement détaché du réel. Le système philosophique se présente comme une belle construction, achevée, mais qui par là même ne peut que rester hermétique à l'intégration de nouvelles données qui risqueraient de mettre en péril la construction tout entière. C'est sur ce point précis que Freud affirme toute la distance entre la philosophie et la psychanalyse. Cette dernière s'attache à résoudre des problèmes concrets en naviguant sans cesse entre la pratique et la théorie, chacune se transformant mutuellement. C'est en ce sens qu'elle se rapproche de la démarche des sciences de la nature. Pour reprendre l'expression d'Assoun «à la clôture philosophique s'oppose l'ouverture expérimentale ». 
C'est particulièrement à cet endroit que l'on regrette qu'Assoun n'ait pas renvoyé à Freud ses propres dires : la psychanalyse ne représente-t-elle pas elle aussi une sorte de totalisation à partir de quelques principes intangibles? Certes Freud n'a pas cessé tout au long de sa vie de remodeler sa théorie, mais le désir d'explication totale des phénomènes psychiques à partir sinon d'un seul principe du moins d'un nombre extrêmement restreint ne persistait-il pas? Par exemple, le fait que tout rêve soit, malgré toutes les difficultés que cela comporte, interprété comme la réalisation d'un désir refoulé, ou le fait que l'étiologie de la névrose soit toujours de nature sexuelle, ne relèvent-ils pas d'un tel désir de totalisation théorique?

Freud - et Assoun développe très clairement ce point - n'est jamais tant menacé par les travers de la philosophie que lorsqu'il tente de concevoir une métapsychologie. En effet, toute science, et sans doute particulièrement la psychanalyse, comprend, à côté de sa partie purement empirique fondée sur l'observation des faits, une partie plus spéculative constituée par les principes directeurs de la science en question. La métapsychologie représente cette dernière. Elle est la part la plus philosophique, et en tant que telle la plus menacée par ces travers. La métapsychologie se distingue des sciences de la nature, notamment de la médecine, par le fait qu'elle ne s'en tient pas à une simple lecture des faits. Elle affirme la nécessité de recourir à des concepts fondamentaux (Grundbegriffe) pour unifier la disparité des faits; pour les faire parler. Or, c'est précisément dans ce recours à un certain nombre de concepts fondamentaux unifiants que la métapsychologie se rapproche dangereusement de la démarche philosophique. Freud introduit alors une distinction, selon nous tout à fait contestable, entre le concept philosophique et le concept métapsychologique : «Le Grundbegriff philosophique traduit l'exigence d'a priorité radicale : il exprime ainsi le projet de déduction universelle à partir de quelques "postulats" ou "présupposés" [Voraussetzungen] 》 (p. 113).

Après avoir analysé les distinctions que Freud établit entre la philosophie et la psychanalyse, Assoun thématise l'interprétation psychanalytique de la philosophie, d'une part en tant que production individuelle, d'autre part en tant qu'institution culturelle.

En effet, comme production de l'esprit humain, la philosophie peut être prise pour objet de l'investigation psychanalytique. La philosophie a alors le statut de symptôme. Cette lecture psychanalytique de la philosophie permet de déterminer les constantes psychologiques de l'auteur, mais surtout, par là même, de déterminer ce qui résiste à ces constantes et constitue alors le noyau purement conceptuel de la pensée, son objectivité, en quelque sorte.

Freud interprète également la philosophie en tant que forme d'activité spécifique de la civilisation. Il en souligne alors la proximité avec cette forme de pensée archaïque: l'animisme. Ces deux formes de pensée ont en commun «la surestimation de la magie verbale " et "l'idée que notre pensée guide et règle les phénomènes ». Et derrière l'animisme, c'est le narcissisme secondaire, cette phase dérivée du narcissisme qui se caractérise par "l'introduction des investissements d'objets », que Freud reconnaît. Le désir de Weltanschauungen, inhérent à la phi- 
losophie, est symptomatique du narcissisme puisqu'il s'agit de reconstituer la totalité du réel à partir d'un principe anthropomorphique. C'est enfin la critique classique de la philosophie comme sublimation qui apparait. En tant que construction artistique, la philosophie est alors interprétée, à l'instar de n'importe quelle œuvre d'art, comme la sublimation de pulsions libidinales.

On le voit, l'analyse de la philosophie en tant que production individuelle et l'analyse de la philosophie en tant qu'institution culturelle, s'éclairent mutuellement. Ce sont sur ces analyses que s'achève la partie principale de l'ouvrage qui nous intéresse ici.

Les deux dernières parties, ainsi que la préface, traitent du rapport de Freud aux philosophes, pris un à un. Ces deux parties ont l'avantage de constituer un recensement exhaustif fournissant un précieux outil de travail. Cependant, le classement par auteur aboutit à une disparité des thèmes abordés qui rend cette partie difficilement résumable. Pour finir, nous pouvons rappeler le grand intérêt de l'analyse conceptuelle de la première partie et le merveilleux outil de travail que fournissent les deux dernières.

Ariane Poulantzas

Theodore M. Porter, Trust in numbers. The pursuit of objectivity in science and public life. Princeton, NJ, Princeton University Press, 1995. $16 \times 24,310$ p., bibliogr., index.

L'analyse historique et sociologique que propose Theodore M. Porter dans son nouvel ouvrage constitue un bel exemple de recherche sur les fondements sociaux des autorités à la fois scientifiques et sociales (administratives) : les dossiers présentés constituent un ensemble historiographique riche et varié. L'auteur propose quelques éléments de réponse voire même « une » réponse à la question des causes de l'expansion de la volonté et des démarches de quantification dans la société, dans les savoirs qui ont trait à cette société mais aussi dans les savoirs sur la nature (sciences sociales mais aussi naturelles). La période couverte s'étale approximativement de 1830 à 1970. Les éclairages fournis par l'auteur sont d'autant plus précieux que l'analyse des contextes sociohistoriques d'apparition et de développement des outils quantitatifs est encore rare dans la littérature d'histoire ou de sociologie des sciences.

Le premier temps de l'argumentation de Porter repose sur la démonstration que le recours à la quantification permet d'échapper au caractère local de toute connaissance, au caractère subjectif de toute décision individuelle, au caractère 
partial de toute opération d'observation. La quantification est donc un outil d'objectivation, un instrument permettant d'assurer l'impartialité des décisions : c'est une technologie de distanciation (technology of distance) permettant de s'éloigner du particulier et de tendre vers le général, l'universel. Porter cite par exemple les efforts de l'industrie pharmaceutique pour standardiser ses produits durant les premières années de notre siècle: l'adoption de normes numériques apparaissait, aux yeux des biologistes, des médecins ou des pharmaciens, comme la promesse de voir leurs mesures accéder au même statut d'universalité que la mesure des longueurs ou des masses, et ainsi s'imposer à la société tout entière.

Sur ce point, Porter étend à une époque contemporaine les propos tenus par Witold Kula dans son riche et utile ouvrage Les Mesures et les hommes (Paris, Éd. de la Maison des sciences de l'homme, 1984) : c'est la nécessité de disposer de conventions uniformes et interpersonnelles lors des transactions marchandes qui a imposé, au fil des siècles, les systèmes des poids et mesures (notamment le système métrique). Le développement de la quantification serait donc lié à l'évolution des relations marchandes entre les hommes et à la nécessité d'administrer leur communauté. Porter nie en quelque sorte l'existence éventuelle d'une approche « naturellement » quantitative des phénomènes. Il s'inscrit explicitement dans la même perspective que Witold Kula lorsque ce dernier affirme que la mentalité primitive appréhende les objets de manière synthétique et qualitative et qu'il a fallu des milliers d'années pour que se constitue une attitude abstraitement quantitative. Porter sépare d'ailleurs l'histoire des disciplines mathématiques en deux branches très distinctes : les mathématiques des nombres (arithmétique, algèbre) qui puisent leurs racines du coté des pratiques marchandes et comptables; et les mathématiques sans nombres (théorie des ensembles, logique, analyse, mathématiques modernes développées depuis le $\mathrm{xvI}^{\mathrm{e}}$ siècle) qui trouvent leurs origines du côté de la géométrie, de la logique, du raisonnement abstrait et formel.

Sur ces divers points, qui constituent l'essentiel de la première partie du livre (p. 9-86), les propos de Porter s'appuient sur la littérature secondaire. Du coup, ils manquent parfois de cohérence et de systématicité. La tâche que se fixe l'auteur est, il est vrai, difficile : au regard de l'étendue des questions et des périodes abordées, les sources secondaires sont rares et hétérogènes. Reste, pour le lecteur, un sentiment d'incomplétude, un sentiment de curiosité déçue. L'autre limite à la portée des propos de Porter se situe dans le choix de sa définition de l'objectivité : toute sa démarche repose sur une définition de l'objectivité qui écarte très explicitement toute référence à la réalité de la nature ou à la vérité des énoncés la concernant. Elle renvoie seulement à l'idée d'indépendance vis-à-vis des circonstances ou des intérêts particuliers. Elle s'oppose directement et simplement à la subjectivité : la définition est, de ce point de vue, négative. Porter écarte explicitement de son analyse toute réflexion sur la réalité, sur les capacités ou les incapacités des savoirs scientifiques et techniques à rendre compte, au moins en partie, de la réalité.

Le recours à la quantification n'est toutefois pas général: seuls les acteurs sociaux dont les capacités d'expertise ne sont pas reconnues, seuls les groupes 
dont l'autorité n'est pas fondée, introduisent l'usage des nombres et des outils quantitatifs dans leurs prises de décision, dans l'exposé de leurs résultats. Ce recours apparaît dès lors comme une stratégie sociale, comme une technologie sociale permettant aux uns et aux autres d'asseoir leur autorité : la quantification permet d'assurer la confiance (trust) dans les choix d'expertise et les résultats d'expériences et, par là, de donner autonomie et assurance aux communautés d'experts. Ce second point de l'argumentation (qui occupe, schématiquement, la deuxième partie du livre, p. 87-190) repose pour l'essentiel sur des recherches empiriques sur des sources primaires : il s'agit, aux yeux même de l'auteur, du cœur de son travail. Celui-ci est comparatif : c'est sur des analyses différentielles des situations des actuaires britanniques du $\mathrm{XIX}^{\mathrm{e}}$ siècle et des comptables américains du $\mathrm{xx}^{\mathrm{e}}$ siècle d'une part, des ingénieurs français du corps des ponts et chaussées au $\mathrm{XIX}^{\mathrm{e}}$ siècle et des ingénieurs militaires américains de notre siècle d'autre part, que Porter fonde l'essentiel de ses développements. Suivons par exemple le cas des ingénieurs français et américains : les ingénieurs d'État du corps des ponts et chaussées comme les ingénieurs du corps militaire américain ont pour charge principale la planification et la réalisation des édifices de génie civil (ponts, voies ferroviaires et routières, barrages notamment). Pour Porter, l'autorité des ingénieurs français, historiquement fondée sur la sélection culturelle, sociale et intellectuelle de ses membres, repose sur son élitisme et sur l'acceptation de cet élitisme par une société française centralisée et hiérarchisée. La reconnaissance de cette autorité permet au corps de prendre des décisions sans devoir en rendre compte publiquement, sans avoir recours à des arguments quantifiés impersonnels. Leur formation en mathématiques et sciences de l'ingénieur n'était pourtant pas un obstacle et aurait même pu constituer une incitation au développement de méthodes quantitatives de décision. À l'inverse, le corps des ingénieurs militaires américains a dû développer une méthode quantitative (l'analyse coût-bénéfice) pour justifier ses propositions. Son autorité, comme celles de toutes les agences gouvernementales américaines, n'était pas suffisamment fondée pour se passer d'un système d'argumentation présentant les signes de l'objectivité. La quantification apparaît comme un instrument permettant aux ingénieurs dont l'autorité n'est pas encore assurée de fonder leur autorité sociale voire scientifique.

Porter tire, jusqu'au bout, les conséquences de sa démonstration (dernière partie, p. 191-231): non seulement les ingénieurs, les comptables et les économistes mais les scientifiques eux-mêmes ont bâti leur légitimité sur le recours à la mesure, à la quantification. L'auteur réfute donc l'idée, largement répandue, selon laquelle les sciences de la nature auraient fourni leur modèle (l'idéal quantitatif) aux sciences de la société : les causes du développement des instruments de mesure (balances, chronomètres, ampèremètres...) ne seraient pas liées aux nécessités «ontologiques » du travail scientifique mais à des contraintes économiques et administratives. La quantification ne s'imposerait donc pas par nature mais par culture, comme conséquence de l'existence nécessaire de règles communes à tous les hommes vivant en société, comme corollaire de l'élaboration d'une science et d'une technologie partagées par le plus grand nombre. Porter illustre ses propos par l'étude du rôle des statistiques inférentielles en médecine et psychologie. 
Pour incarner cette thèse, la figure de Karl Pearson est avancée à de nombreuses reprises par Porter. Il voit chez le savant anglais un exemple de scientifique ayant développé une conception de la science bâtie sur la volonté d'administrer le monde plus que de le comprendre, sur une philosophie davantage sociale que naturelle, sur la recherche d'instruments permettant de subordonner les intérêts particuliers à des règles générales. À la figure de Karl Pearson nous pourrions opposer celles de beaucoup d'autres incarnant une conception réaliste des mesures. Citons par exemple Galton, Weldon ou Spearman qui se sont opposés à certaines interprétations de Pearson. Comment les nombreuses controverses sur le réalisme des indicateurs quantifiés ou des méthodes quantitatives s'intègrent-elles à la thèse de l'ouvrage?

Au total et au-delà des limites et critiques que le travail de Porter appelle, la thèse défendue a incontestablement une réelle pertinence qui devra, au fil des investigations futures, être précisée et circonscrite (dans le temps et l'espace disciplinaire). L'ouvrage a la vertu de proposer, dans un champ de recherche riche et encore largement vierge, une thèse forte qui devrait susciter des réactions. L'auteur ne le cache pas : il y voit même une des premières ambitions de son livre.

Olivier MARTIN

Émile Meyerson, De l'explication dans les sciences. Rééd. Paris, Fayard, 1995. $13,8 \times 22,3,979$ p. ( (Corpus des œuvres de philosophie en langue française »).

Le moment est venu d'apprendre à connaître l'œuvre d'Émile Meyerson. Longtemps, son seul titre de gloire fut de rassembler tous les vices de l'épistémologie pré-bachelardienne. On oubliait alors facilement que l'ami de L. Brunschwig, L. Lévy-Bruhl, P. Painlevé et $\mathrm{H}$. Høffding avait eu un rôle décisif dans la formation d'historiens des sciences comme A. Koyré et H. Metzger ${ }^{3}$; on ramenait une pensée souvent subtile et toujours érudite à quelques slogans aisément stigmatisables par des mots en "-isme»: substantialisme, chosisme, choquisme, réalisme (naïf), continuisme; on condamnait sans appel l'ambition d'élaborer une théorie universelle de la connaissance. Assurément, certains aspects de cette œuvre nous sont aujourd'hui devenus étrangers; il me semble cependant nécessaire d'en reconnaitre l'originalité, la valeur et la fécondité.

3. Il suffit à vrai dire de lire les Études galiléennes sans préjugé pour noter les hommages appuyés d'A. Koyré à É. Meyerson; Ernest Coumet, dans son article "Alexandre Koyré : la Révolution scientifique introuvable? », History and technology, $\mathrm{n}^{\circ}$ spéc., "Proceedings of the International Conference Alexandre Koyré », vol. 4, 1987, p. 514-524, montre plus précisément que les difficultés historiques qu'É. Meyerson avait rencontrées dans l'analyse du principe d'inertie expliquent en partie le programme de recherches d'A. Koyré. Les questions que pose Hélène Metzger à l'histoire de la chimie sont elles aussi inspirées par l'épistémologie d'É. Meyerson (sur ce thème, voir, par ex., Jan V. Golinski, « Hélène Metzger and the interpretation of seventeenth-century chemistry », History of science, 25, 1987, p. 85-97). 
Publié quatorze ans après Identité et réalité, De l'explication dans les sciences en constitue « une suite, ou plutôt un complément ${ }^{4}$ »; ce n'est pas exactement une suite car les thèses fondamentales sont les mêmes d'un ouvrage à l'autre; c'est plutôt un complément car, en se tournant vers le domaine encore inexploré de la philosophie de la nature hégélienne, le second ouvrage confirme et approfondit les thèses qu'avait fait naître dans le premier l'analyse de la science au sens strict. De l'explication dans les sciences se compose en effet de quatre livres: les deux premiers reprennent les thèses d'Identité et réalité en les présentant autrement ${ }^{5}$; le troisième étudie, et cela est nouveau, la tentative hégélienne d'expliquer globalement la nature; le quatrième enfin compare la raison scientifique et la raison philosophique. Entrons maintenant plus avant dans le détail du développement.

Les deux thèses que Meyerson oppose au positivisme d'A. Comte et au phénoménisme d'E. Mach font l'objet du premier livre. La science a en premier lieu une visée ontologique : elle construit des choses, c'est-à-dire des réalités indépendantes des sensations et plus durables qu'elles; la science d'aujourd'hui détruit assurément les choses du sens commun et de la science d'hier, mais c'est toujours au profit de nouvelles choses. En second lieu, la science n'est pas seulement légale : elle ne se contente pas d'établir des rapports entre phénomènes, mais cherche à expliquer les phénomènes, à en trouver la cause.

Le deuxième livre examine conséquemment la «marche de l'explication », son enjeu et ses limites. Expliquer un phénomène, c'est en trouver la cause, le réduire à une conséquence logique, l'inscrire dans une chaîne déductive; la force explicative d'une théorie physique ne vient pas en effet de ses principes - ils sont toujours obscurs - mais des enchaînements déductifs qu'elle rend possibles. L'explication scientifique suppose implicitement la rationalité du réel; or ce qui caractérise en général notre raison, c'est le processus d'identification; expliquer consiste dès lors à identifier des phénomènes qui paraissent divers dans l'espace ou dans le temps. C'est alors qu'intervient le concept fondamental de l'irrationnel « qui excite un intérêt tout à fait dramatique ${ }^{6} »$. Est irrationnel en général selon Meyerson tout ce qui résiste à la raison dans sa démarche identificatrice, autrement dit tout ce qui apparaît comme un donné ou comme un divers irréductible : la sensation comme telle, la qualité, le proprium quid des phénomènes, leur diversité dans l'espace et leur irréversibilité dans le temps, mais aussi les principes ou les hypothèses propres à chaque science. De l'explication dans les sciences approfondit une théorie de l'irrationnel encore imprécise dans Identité et réalité : les irrationnels varient bien sûr selon les sciences dont ils manifestent la spécificité - mathématique, et surtout

4. É. MeYerson à H. HøғFding, sans date [entre mai et novembre 1918], Correspondance entre Harald Høffding et Émile Meyerson, publiée par Frithiof BRANDT, Hans HøFDING et Jean AdigARD de GaUTRIEs, Copenhague, Einar Munksgaard, 1939, p. 4.

5. Voir préf., p. 9-10: « Nous avions, autrefois, traité le schéma d'identification comme un énoncé simplement heuristique [...] Il semble que cette manière d'exposer la thèse [...], entièrement a posteriori, $[\ldots]$ ait dérouté plus d'un lecteur $[. .$.$] C'est pourquoi nous nous sommes$ demandé s'il n'était pas possible de parvenir au même résultat par une marche plus proprement logique, en essayant de démontrer plus directement le mécanisme de la pensée scientifique. $"$

6. H. HøFfding à É. MeYerson, 29 avril 1921, op. cit. supra n. 2, p. 6. 
physique, chimie et biologie - , mais aussi selon leur situation par rapport à ces sciences - le mécanisme exclut par exemple la sensation comme telle, mais prend le choc comme fondement de toutes ses explications - , enfin selon leur degré d'élaboration - le principe de Carnot a délimité l'irrationnel qu'est l'irréversibilité temporelle ${ }^{7}$, mais la diversité dans l'espace n'a pas encore trouvé d'expression définitive. L'idée directrice de Meyerson est qu'on ne peut assigner à résidence l'irrationnel une fois pour toutes, car il surgit toujours inopinément dans la science, là où il n'était pas attendu, sous une forme qu'on n'avait pas prévue. La fin de ce deuxième livre apporte des précisions sur les modalités et les possibilités futures des explications spatiales, puis montre que les notions de puissance ou de potentiel fournissent un succédané d'explication là où l'identification ne réussit pas immédiatement.

Le troisième livre porte sur la philosophie de la nature de Hegel : cela surprendra de la part d'un épistémologue car cette partie du système hégélien est un tissu d'aberrations; cela était d'autant plus étonnant en 1921 qu'elle avait été négligée par les commentateurs ${ }^{8}$.

Meyerson estime cependant le cas hégélien exemplaire pour confirmer ses thèses sur la nature de la raison et spécifier la différence entre la voie qu'elle emprunte dans les sciences et dans la philosophie. Les raisons de l'échec de Hegel sont aussi celles de sa grandeur : il a entrepris de montrer immédiatement la rationalité effective du réel dans sa totalité ; il est parti à cet effet des sensations que le mécanisme exclut, sans comprendre la puissance de la déduction mathématique; surtout, il a prétendu rationaliser l'irrationnel en le réduisant à une seule espèce, l'être-autre or la richesse des sciences vient précisément de ce qu'elles admettent différents irrationnels au fondement de leur déduction. À l'analyse interne de l'œuvre succède sa confrontation avec les démarches de Schelling, Comte, Descartes et Kant.

Dans le quatrième livre, Meyerson expose pour conclure sa théorie de la connaissance. La science ne peut se passer d'ontologie, mais, dans son exercice même, elle est indifférente à toute métaphysique particulière. Les démarches scientifiques et philosophiques aussi bien que celles du sens commun sont fondées sur la recherche de l'identité; le succès des sciences montre qu'il existe un accord partiel entre notre raison et le réel; le processus d'identification est le moteur de toutes les sciences, ce qui explique qu'elles ne consistent jamais à enregistrer des faits bruts, mais se présentent bien au contraire comme des théories déductives. Si le schéma identitaire est d'une telle importance, comment se fait-il cependant qu'on ne s'en soit pas aperçu

7. C'est l'occasion de rappeler le profond jugement qu'avait porté Léon BRUNSCHvicG, dans son article « La philosophie d'É. Meyerson », Revue de métaphysique et de morale, 33, 1926, p. 40, sur Identité et réalité : «L'apologie de la causalité identificatrice ne sert qu'à dresser le piédestal sur lequel s'élèvera la statue du véritable héros du livre, qui est Sadi Carnot. "

8. É. Meyerson à H. HøfFding, 5 sept. 1921, op. cit. supra n. 2, p. 11 : «Je suis particulièrement heureux de ce que vous approuvez ce que j'ai exposé au sujet de la philosophie de Hegel. Je vous avoue que j'éprouvais, de ce chef, des craintes assez vives. Par le fait même que je me suis occupé d'un côté de sa philosophie que ses disciples ont généralement négligé, l'image que Hegel prend chez moi n'est pas tout à fait conforme à la tradition. » Voir sur ce point, Alexandre KoYRé, « Rapport sur l'état des études hégéliennes en France », Verhandlungen des ersten Hegelkongresses, Tübingen, 1931, repris in ID., Études d'histoire de la pensée philosophique, Paris, Gallimard (« Bibliothèque des idées »), 1971, p. 236-241. 
plut tôt? Il y a à cela une raison profonde : il est difficile d'admettre que la raison poursuive obscurément un but absurde, la négation du divers dont elle a pourtant besoin pour s'exercer et sans lequel le monde serait inexplicable. L'ultime chapitre affirme l'unité de la raison humaine tout en cherchant à maintenir la spécificité de la philosophie et de la science; le verdict semble cependant rude pour la philosophie : elle ne peut, comme la science, admettre l'existence d'irrationnels sans se compromettre et, si elle les refuse, elle est vouée au destin monstrueux des philosophies de la nature; elle doit baisser les armes devant les résultats scientifiques; elle a pour seule fonction de faire connaître au scientifique les acquis du passé.

Voici ramené à quelques lignes un livre qui avoisine les mille pages; ce résumé pour le moins succinct ne montre guère en quoi De l'explication dans les sciences est l'écrit d'un philosophe et d'un historien des sciences : j'ai dû négliger les arguments, les nuances et les précautions qui accompagnent toutes les thèses de Meyerson, mais aussi la multitude de références à l'histoire des sciences qui les étayent. Parfois même, le travail de l'historien semble prendre le pas sur la tâche du philosophe : l'ouvrage s'achève par vingt et un appendices qui sont autant de mises au point historiques n'ayant pas trouvé leur place dans le développement principal. J'espère avoir du moins éveillé la curiosité des lecteurs; je finirai en tout cas comme j'ai commencé : le moment est venu d'apprendre à connaître l'œuvre de Meyerson.

Sophie Roux

«Les sciences biologiques et médicales en France, 1920-1950 », actes du colloque de Dijon, 25-27 juin 1992, éd. Claude Debru, Jean GaYon, Jean-François PICARD, Cahiers pour l'histoire de la recherche, 1994.

Ces actes qui présentent les travaux historiques de spécialistes français et étrangers viennent combler une grave lacune dans le domaine de l'histoire des sciences biologiques en France. Premier ouvrage consacré à ce sujet, il constitue une source d'information irremplaçable. Dans ce recueil sont abordés des thèmes qui dans leur diversité laissent apparaître en filigrane deux questions transversales: celle de la pertinence de la notion de « tradition nationale » en sciences, et celle de la « stagnation des sciences biologiques françaises » au cours de la période étudiée. Ces deux questions ne sont pas obligatoirement liées : il arrive que des chercheurs travaillant dans un pays donné soient à ce point mal insérés dans la communauté scientifique internationale qu'ils deviennent stigmatisés comme représentants d'une science «stagnante » par rapport aux acquis de cette communauté internationale, sans avoir pour autant développé un «style national » distinct. Une telle «stagnation » peut se développer en particulier dans les pays périphériques, coupés des grandes centres de recherche. D'autre part, une forte tradition scientifique nationale - tel l'héritage de la physiologie de Claude Bernard dans la biologie française - peut à la fois favoriser la cristallisation d'un «style national » et rendre plus difficile l'intégration des chercheurs français dans les circuits internationaux. Les éditeurs des «Sciences 
biologiques et médicales en France " s'interrogent en premier lieu sur la réalité d'un «style français » capable de produire des recherches biologiques menées entre 1920 et 1950; en second lieu sur la validité de l'opinion qui veut que cette période corresponde à une «stagnation" des sciences biologiques en France, ou plutôt au développement des directions d'investigation différentes, enfin sur le lien possible entre ces deux problématiques.

Les cas étudiés dans ce volume sont loin de fournir une réponse claire aux questions posées par les organisateurs du colloque de Dijon. Ils suggèrent cependant un certain nombre de pistes pour l'avenir. Dans leur majorité, ces études de cas confirment tout à la fois l'existence d'une certaine « spécificité » de la biologie française et sa «stagnation" - ou plutôt sa différence - dans la période étudiée. Abordant ces notions de façon nuancée, elles pointent l'existence d'importantes variations entre sites, entre domaines de recherche, et même entre les situations dans lesquelles se trouvent les chercheurs engagés dans des investigations relativement proches. Ainsi, certains domaines d'étude directement inspirés par la physiologie générale - l'étude du comportement animal analysé par Richard Burkhardt, ou la théorie d'excitabilité de Louis Lapicque étudiée par Joy Harvey et par Jean-Claude Dupont - n'ont guère bénéficié de l'essor qu'ils ont connu dans d'autres pays. En revanche, et bien que relevant d'une inspiration physiologique voisine, la protistologie développée par Edouard Chaton a pu donner naissance aux théories fructueuses qui furent reprises par la suite par son élève, André Lwoff, pour être incorporées dans la biologie moléculaire naissante. La relative «stagnation » de la majorité des recherches physiologiques contraste encore avec la vitalité d'un petit groupe de chercheurs en embryologie expérimentale étudié par Richard Burian, Michel Delsol, Janine Flatin, Jean-Louis Fisher, Jean Gayon et Hervé Le Guyader.

La diversité des cas étudiés dans ce volume confirme le danger des généralisations abusives. Elle n'élimine cependant pas le besoin d'une vision plus large. En dépit de l'intérêt indéniable des problèmes posés par les cas particuliers qui composent «Les Sciences biologiques et médicales en France », on peut regretter que ce volume n'ait pas inclus des travaux à caractère plus synthétique, et que les auteurs aient eu des difficultés à aller au-delà des pistes partielles indiquées dans les recherches ponctuelles. Il s'agit là, on peut le supposer, d'une contrainte propre à la forme "actes d'un colloque ». L'introduction avertit que "l'histoire des sciences biologiques et médicales en France entre 1920 et 1950 nécessiterait, pour être véritablement écrite, la maîtrise d'un matériel considérable qui reste encore à rassembler, et l'invention de méthodologies nouvelles adaptées à l'énormité de ce matériel » (p. 12). On peut cependant imaginer des recherches qui se situeraient à mi-chemin entre l'étude aboutie d'une période entière et une série d'études de cas isolés, et des travaux qui feraient des cas réunis ici le point de départ de nouvelles recherches prenant en compte, sur une période plus longue, davantage de protagonistes et de sites.

Les éditeurs du recueil affirment à juste titre que « les explications intellectuelles, conceptuelles, techniques, institutionnelles, démographiques de l'histoire des sciences ne sont pas exclusives les unes des autres », et que la combinaison de plusieurs démarches partielles améliorera la compréhension du développement des sciences biologiques en France. Cependant, à l'exception des travaux consacrés à la biologie appliquée et à la recherche médicale centrés sur les développements institu- 
tionnels, les recherches réunies dans ce recueil s'intéressent avant tout aux questions conceptuelles et méthodologiques. Cette polarité dans le traitement des sujets risque d'induire l'impression que les aspects institutionnels et techniques deviennent moins importants dès qu'on passe à l'histoire des recherches fondamentales en biologie. Des études à visée synthétique axées sur des systèmes expérimentaux, des écoles de pensée, des cultures de laboratoire, des institutions scientifiques, sur la place des sciences biologiques et médicales dans l'imaginaire collectif de la nation (par exemple le culte des " grands savants ", les cérémonies commémoratives, l'histoire des disciplines écrite d'un point de vue national) peuvent faciliter l'articulation des divers points de vue sur l'histoire d'une science. De telles recherches pourraient être le sujet d'un futur volume qui complétera cet ouvrage.

Ilana LöwY

\section{QUESTIONS DE SCIENCES SOCIALES}

Sciences et langues en Europe. Sous la dir. de Roger Chartier \& Pietro Corsi. Paris, École des hautes études en sciences sociales, 1996. 16,8 $\times 24,273 \mathrm{p}$. ( Commission européenne-Forum européen de la science et de la technologie »).

Le sujet de ce volume, qui fut d'abord celui d'un colloque (Paris, nov. 1994), prête souvent aux excès, aux débats passionnés, aux descriptions apocalyptiques, aux protestations solennelles, quand il ne se dilue pas dans des généralités pompeuses. Or, la première impression que l'on retire de la lecture de ce recueil est celle d'une grande justesse de ton. Si le parti historique qui domine dans la plupart des textes explique largement cela, la même remarque vaut pour les quelques communications qui portaient sur, dirions-nous, la pratique, au premier rang desquelles le texte fort stimulant de Jean-Marc Lévy-Leblond.

Le programme scientifique du colloque amenait à réfléchir sur les rapports entre les sciences et les langues, en Europe, de la Renaissance au temps présent. Trois axes de recherche se présentaient, en fait trois couples d'opposition entre d'une part, les langues vernaculaires et, d'autre part, une langue universelle, une langue parfaite, une langue véhiculaire. Le plan suivant lequel les dix-sept communications ont été ordonnées, rend assez bien compte des questions qui ont été posées : 1. Latin et langues vernaculaires dans l'Europe de la première modernité; 2 . Langues vernaculaires, langues universelles aux XVI $I^{e}$ et XVII siècles; 3 . De la langue « naturelle " à 
la convention linguistique; 4 . La langue de la science $:$ les dimensions linguistiques; 5. La langue des sciences aux $x^{e}{ }^{e}$ et $x^{e}{ }^{e}$ siècles; 6 . Enjeux et débats dans le monde contemporain.

Plutôt que de donner un résumé analytique de chaque texte, il nous est apparu préférable de mettre en évidence un certain nombre d'acquis. Tout d'abord, la permanence d'une question de la langue dans la période considérée : pendant quatre siècles, les « savants » se sont, en effet, interrogés - ou ont été amenés à le faire sur la langue à utiliser dans leurs travaux. En cela, une double nécessité est apparue : quelle langue employer pour communiquer au mieux avec autrui, mais aussi pour traduire le plus exactement possible, en toute transparence, le résultat des recherches. Nombre de langues artificielles sont nées de ce second impératif. Ressort aussi de plusieurs communications, le rôle important que le latin a eu dans ce domaine : langue par excellence des sciences, langue véhiculaire de convention, référence et modèle, voire source de certaines langues artificielles des $\mathrm{XIX}^{\mathrm{e}}-\mathrm{xx}^{\mathrm{e}}$ siècles; pour cette dernière période, on se doit de citer l'article d'Anne Rasmussen, l'un des plus solides du recueil, tant par la documentation mise en œuvre que par la finesse de l'analyse. En troisième lieu, l'incidence de phénomènes extralinguistiques apparaît avec netteté dans ce rapport entre sciences et langues, qu'il s'agisse de facteurs politiques et économiques, qu'il s'agisse de considérations d'ordre idéologique, tel le prestige dont jouit une langue, les qualités dont on la pare, l'aptitude qu'on lui reconnaît - ou qu'on ne lui reconnaît pas - à être le véhicule du savoir. Dans cet ordre d'idées, on lira avec intérêt plusieurs textes s'interrogeant sur la situation contemporaine et, traitant non seulement du statut de l'anglais, mais encore du rapport des autres langues avec ce nouveau latin. Enfin, des communications insistent sur le rapport qui existe entre une langue et le processus scientifique luimême, quand la langue n'est pas seulement un véhicule, mais aussi un instrument d'invention : la contribution de Marco Beretta qui porte sur la nomenclature de la chimie au XvIII siècle est fort éclairante de cette conception de la langue qui est alors pour le savant, et la citation est empruntée à Lavoisier, "une véritable méthode analytique ».

À côté de ces acquis, ce volume d'une haute tenue offre bien des suggestions et présente des pistes de recherche à poursuivre : en particulier, la dimension orale du savoir. Les quelques remarques d'Anne Rasmussen sur l'importance des colloques dès la fin du XIX ${ }^{e}$ siècle, de Jean-Marc Lévy-Leblond et de Geoffrey Nunberg sur la spécificité des échanges informels dans le monde scientifique contemporain invitent à ne pas cantonner les enquêtes au seul domaine de l'imprimé. Pour en rester à l'Ancien Régime et au xix ${ }^{e}$ siècle, la rencontre savante, le cours universitaire, la séance académique constituent des situations des plus fréquentes dans le monde intellectuel, situations que des documents d'ordre narratif permettent de saisir dans leur dimension orale. Au titre des regrets, on constatera l'équation implicite qui a été posée entre sciences et sciences « dures », excluant d'emblée le champ de l'histoire, de la philosophie, bref, de ce que l'on appelle les sciences humaines. De même, on soulignera l'absence d'intérêt pour l'une des formes linguistiques que la 
science - toutes disciplines confondues - n'a jamais ignorée : le jargon ${ }^{9}$. Peutêtre est-ce parce que les auteurs de ces textes s'en sont dans l'ensemble soigneusement gardés ${ }^{10}$.

Françoise WAQUET

Jacques Proust, L'Europe au prisme du Japon, xví-xvilf siècle. Entre humanisme, Contre-Réforme et Lumières. Paris, Albin Michel, 1997. 14,5 × 22,5, 319 p., bibliogr. («Bibliothèque Albin Michel Histoire»).

Jacques Proust ne nous entretient pas dans cet ouvrage de l'Europe de l'époque moderne qui nous est familière, mais de l'Europe telle qu'elle fut perçue et imaginée par les Japonais cultivés, du XvI ${ }^{e}$ au XvIII ${ }^{e}$ siècle. Le prologue qui précède les sept chapitres du développement en éclaire l'esprit : il ne s'agit pas ici de reprendre le cheminement traditionnel d'une histoire des idées s'attachant à établir le bilan d'une influence européenne sur la culture japonaise. Deux défauts majeurs entravent une telle démarche : croire que l'Europe exporte une image authentique et complète d'elle-même, croire aussi que l'autre ne peut choisir qu'entre une acceptation globale et sans réserve d'une telle image et son rejet pur et simple. L'européocentrisme fréquent de l'histoire des idées a ainsi condamné les historiens à ne voir dans l'accueil fait par les étrangers à la culture européenne qu'un miroir plus ou moins fidèle alors que la complexité des modalités de connaissance et des réactions s'apparente plus à une reconstruction de l'image qu'à un simple reflet passif. Cette conception des rapports culturels entre Orient et Occident n'est pas tout à fait nouvelle : elle sous-tendait déjà l'étude de Jacques Gernet parue il y a quelques années sur la Chine et le christianisme. Mais la méthode est ici différente : Jacques Gernet ${ }^{11}$ avait mis en œuvre sa connaissance de la langue chinoise où la transcendance n'est pas exprimable pour mettre en lumière le malentendu profond et inévitable entre missionnaires et Chinois. La démarche de J. Proust est différente : elle s'appuie sur

9. Sur les jargons, on signalera l'ouvrage extrêmement stimulant, publié sous la dir. de Peter BURKe et de Roy PORTER, Languages and jargons. Contributions to a social history of language, Cambridge, Polity Press, 1995.

10. Toutefois, on lit, p. 129: «La science, en effet, est une création humaine, elle est une production humaine de part en part, une production réalisée avec les moyens du bord par des hommes, écrite et parlée par des femmes [...] »; cette phrase est accompagnée de la note suivante: "Je propose d'employer alternativement hommes et femmes pour signifier "humains" ". On doit se rappeler l'explication ainsi fournie pour éviter à la page suivante de penser à une division du travail suivant les sexes : « [...] ce sont les hommes qui s'expriment et nous racontent ce qu'ils tiennent pour sa voix [de la nature], ce sont les femmes qui la singularisent pour la maîtriser; ce sont eux qui la manipulent de toute (sic) les façons [...] pour en dire le vrai. Et ces femmes parlent dans une langue localement située, toujours lourde d'une polysémie non maîtrisable [...] ». Le lecteur jugera de cet égalitarisme d'inspiration politiquement correcte.

11. Jacques Gernet, Chine et christianisme. Action et réaction, Paris, Gallimard (« Bibliothèque des histoires »), 1982. 
les textes accessibles en des langues occidentales et sur des œuvres figurées qui révèlent l'image que l'Europe a donnée d'elle-même et la façon dont elle a été perçue.

Les quatre premiers chapitres sont consacrés à la période portugaise : de l'arrivée des premiers jésuites au déclenchement de la persécution des chrétiens en 1614. Période marquée par la présence missionnaire et par un événement emblématique : le voyage de quatre jeunes catéchumènes japonais en Europe sous la férule de leurs maîtres jésuites, sujet du dialogue du De missione legatorum, publié en 1590 à Macao sous la direction d'Alexandre Valignano, alors visiteur de la Compagnie au Japon. De Rome à Coïmbra, l'Europe donnée à voir aux jeunes visiteurs et lecteurs éventuels est celle d'un catholicisme triomphant, unanime, même s'il est teinté d'humanisme. C'est également cette image que véhiculent des ouvrages imprimés par les pères de la Compagnie sur leurs presses japonaises ou demeurés manuscrits $^{12}$ : catéchismes, exposés de la doctrine chrétienne, histoire du Japon à l'usage des Européens. Mais le message religieux des pères est fortement déterminé par l'enseignement scolastique et les catégories aristotéliciennes : il repose sur la notion d'être, étrangère à la philosophie japonaise, imparfaitement traduite. Aussi la tradition nippone ne retient-elle véritablement des ouvrages européens que les fables d'Ésope, traduites, adaptées et très rapidement assimilées à la culture nationale.

Réticents face aux volontés missionnaires, les Japonais sont beaucoup plus attentifs aux domaines qui échappent à l'emprise de l'autorité et du dogme. C'est ainsi que la médecine et l'art sont les canaux privilégiés des échanges. La médecine en particulier, domaine souvent suspect aux yeux des missionnaires, est dans un premier temps un terrain privilégié de rencontre entre les deux cultures, comme en témoigne l'école de médecine occidentale établie par Luis de Almeida à Funai et qui fonctionne de 1546 à 1593 : la chirurgie occidentale y est enseignée, mais la médecine interne y relève toujours de la tradition chinoise. Quelques années plus tard, le personnage de Cristovão Ferreira est lui aussi représentatif du rôle joué par la médecine. Entré dans la compagnie de Jésus à Lisbonne, il est envoyé de Macao au Japon dès 1609. La grande persécution l'oblige à continuer son apostolat dans la clandestinité; capturé et supplicié, il apostasie. Une fois devenu Sawano Chuan, il s'initie à la médecine auprès des médecins de la Compagnie hollandaise des Indes orientales à Nagasaki et rédige quelques années plus tard un ouvrage de chirurgie.

Homme des marges et des contrats, Ferreira révèle également les failles d'une Europe que les missionnaires présentent unie dans l'attachement au dogme. La réfutation du christianisme rédigée après son apostasie confond les textes bibliques par des arguments de bon sens, souligne les contradictions entre la Bible et l'enseignement d'Aristote et du pape. Quant à sa critique de l'usage des sacrements, elle révèle, souligne J. Proust, qu'il a été "frotté, sinon de "luthéranisme", du moins d'un zeste d'érasmisme» (p. 85). L'expérience missionnaire au Japon amène les jésuites à s'interroger sur les croyances et les coutumes japonaises, mais les entraîne aussi dans une réflexion sur l'utilisation des images dans le culte, réflexion qui

12. En part., l'Historia de Japan du père L. FroIs, publiée en partie en français sous le titre : Traité de Luis Frois, S.J. (1585) sur les contradictions de mours entre Européens et Japonais, préf. José Manuel Garcia, notes et commentaire de Robert ScHRIMPF, Paris, Chandeigne (« Magellane »), 1993. 
tient une part importante chez le casuiste Vasquez. La prédication repose ainsi largement sur l'utilisation des images mais s'adapte au contexte japonais en évitant par exemple un trop grand réalisme dans la représentation de la crucifixion, peine infamante au Japon. Cet art qui favorise les échanges et les contacts est aussi le terrain privilégié des glissements, réadaptations et réinterprétations, les codes culturels ayant présidé à leur élaboration étant ignorés ou mal interprétés. C'est ainsi qu'un paravent japonais représente une bataille de Lépante étrangement terrestre où s'égarent des éléphants venus d'une image modèle de la bataille de Zama, tandis que Philippe II revêt les emblèmes d'un empereur romain.

À partir de 1614 et de la Grande Persécution, le Japon échappe aux ambitions missionnaires des Européens. Dès lors, le prisme japonais ne renvoie plus de l'Europe qu'une image singulièrement éclatée. Jusqu'au $\mathrm{XIX}^{\mathrm{e}}$ siècle, les chrétiens japonais conservent l'essentiel du dogme chrétien à travers le récit oral des Commencements du Ciel et de la Terre, analysé dans le chapitre v. Ce conte apparaît comme le miroir déformé de la prédication : Marie se confondant parfois avec la déesse de la miséricorde, tandis que le Christ est un « Bouddha vivant ». Cependant, ce syncrétisme évite des interprétations trop directement inspirées du shintoïsme ou de l'amadisme dont les ressemblances avec certains aspects du christianisme auraient pu favoriser les confusions.

De 1614 à la fin du XVIII siècle, au cours de la période hollandaise, à laquelle sont consacrés les deux derniers chapitres, c'est à nouveau à travers l'art et les sciences que le Japon emprunte à l'Europe, par le biais d'œuvres mineures. C'est en effet en recopiant des gravures illustrant des livres européens que les artistes japonais introduisent la perspective dans les « images du monde flottant ». Mais cette copie n'est jamais totalement passive et s'accompagne d'une réinterprétation comme cette scène de théâtre où l'artiste a utilisé les lois de la perspective linéaire mais en multipliant les points de fuite, faisant ainsi de l'ensemble de l'œuvre une sorte de kaléidoscope où chaque scène participe à l'animation du théâtre. Cette perspective s'impose au Japon, comme elle l'a fait plus tôt en Europe, par le biais des artistes, avant d'être codifiée par le langage scientifique.

Ce dernier s'introduit également au Japon grâce aux livres de médecine. C'est parce qu'ils sont frappés du décalage entre les planches d'anatomie chinoises et les représentations occidentales que des médecins japonais effectuent les premières dissections au xvIII siècle. Elles leur révèlent l'importance de l'expérience et les amènent à corriger les planches des ouvrages européens et à les traduire en japonais, inventant ainsi une nouvelle langue scientifique, à partir des racines chinoises. $\mathrm{La}$ perspective et l'anatomie font pénétrer le Japon de plain-pied dans le mọnde de la modernité.

Proust rappelle les facteurs qui ont selon lui favorisé une telle progression, en particulier le contact privilégié du Japon avec des auteurs européens qui entretiennent une certaine distance avec le dogme et une orthodoxie trop rigide d'une part, d'autre part le scepticisme relatif de certains japonais cultivés qui ont conscience de « la nocivité absolue de la tradition contre laquelle se battait au même moment l'intelligence européenne : la scolastique aristotélicienne, le principe d'autorité, le dogmatisme » (p. 255). On aura reconnu ici les cibles privilégiées des Lumières, de Diderot et de l'Encyclopédie dont Proust a donné, il y a quelques années, une étude magistrale. Tout en étant profondément original cet ouvrage rejoint les études 
contemporaines menées sur les contacts entre l'Europe et l'Asie, en particulier la Chine. Il contribue à la réflexion menée sur ce qu'une culture parvient à transmettre de sa propre image et au rôle des intermédiaires culturels. Surtout, il attire l'attention sur le fait que ce qui est transmis n'est pas toujours ce qui était pensé comme essentiel, mais ce qui ne devait en être à l'origine que le vecteur ou un aspect mineur : la perspective dans l'art et non le sujet, la médecine et non la religion ${ }^{13}$. Ainsi, non seulement la distance entre les cultures déforme l'image proposée, mais elle contribue également à en renverser la construction et les propres codes de valeur.

Isabelle BRIAN

Olivier Christin, La Paix de religion. L'autonomisation de la raison politique au xvf siècle. Paris, Seuil, 1997. 13 × 21,5, 333 p., bibliogr. (« Liber»).

L'essai d'Olivier Christin analyse un processus longtemps en échec, celui de la coexistence pacifique entre confessions dans le royaume de France et le Saint Empire au cours de la seconde moitié du xvI siècle. Ce faisant, l'auteur place au cœur de son interrogation un moment de l'histoire de l'Europe médiane - en particulier les années 1555-1567 - et une procédure, la « paix de religion » largement oubliée par l'historiographie récente des guerres de religion.

L'irruption du luthéranisme et du calvinisme crée une situation politico-religieuse inédite dans les pays de l'Europe médiane. Aux Pays-Bas, dans le Saint Empire romain de langue germanique, les cantons suisses et le royaume de France, communauté religieuse et corps politique cessent alors de coïncider. La religion du Prince n'est plus partagée par tous ses sujets et la légitimation symbolique de son pouvoir par la religion devient étrangère, voire étrange aux yeux d'une partie de ses régnicoles. Rapidement, cet état de faits paraît irréversible. Les luttes conduites contre les «dissidents » tournent à la confusion de leurs instigateurs, les débats et les colloques religieux animés par des théologiens afin de résorber le fossé entre les thèses de la papauté et des réformateurs échouent, confirmant de profondes divergences dogmatiques. La fracture religieuse est officialisée; dans cette béance, des pans entiers de la société s'engouffrent et se livrent une guerre sans merci, alimentée par des motifs existentiels, confessionnels, politiques ou banalement matériels. Les pays basculent dans la guerre civile, considérée comme la plus singulière, la plus injuste, la plus inhumaine de tous les conflits. Dans ce contexte, marqué par l'insoutenable de la guerre civile, le risque d'un évanouissement du pouvoir souverain et la transmutation de la tolérance religieuse en concorde civile comme l'a exposé Mario Turchetti $^{14}$, des hommes d'État et des théoriciens décident de proposer la pacification intérieure comme réponse à la division religieuse.

13. Plusieurs études actuelles portent notamment sur la musique européenne en Chine : les œuvres du jésuite Joseph Amiot ou du lazariste Pedrini.

14. Mario TURCHETTI, « Religious concord and political tolerance in sixteenth-and-seventeenth-century France », The Sixteenth-Century Journal, XXII, 1991, p. 15-25. 
À situation inédite, solution inédite. Les «paix de religion »- paix de Cappel (1531), paix d'Augsbourg (1555), édit d'Amboise (1563) - sont des textes sans antécédent. Brefs, rédigés en termes généraux, souvent flous, ils prennent acte du partage confessionnel et veulent en neutraliser la charge belliqueuse potentielle. Ils se signalent par une teneur laïcisée étrangère aux interrogations dogmatiques, à l'organisation des Églises, une dimension contractuelle et réclament l'intervention du pouvoir central - l'État - chargé de veiller à leur respect. Pris sous la contrainte de l'événement, d'abord pensés et présentés comme provisoires dans l'attente d'une réunification religieuse, ces textes prêtent le flanc à la critique théorique et voient leur mise en application sujette à d'innombrables entraves. Toutefois, il y eut grâce à la volonté du pouvoir souverain relayée dans le pays par des commissaires et la détermination des exécutifs citadins, la mise en pratique de "paix de religion». Les édits généraux de pacification devenaient la matrice des protocoles locaux, reposant sur quelques solides fondements tels que le principe de la liberté de conscience, l'acceptation de la coexistence confessionnelle et sa mise en pratique. Au quotidien, l'expérimentation de la différence religieuse et l'existence d'une minorité et d'une majorité confessionnelle aboutissent à une variété de solutions. Christin, à l'aide d'exemples allemands, helvétiques et de villes du Sudest de la France, distingue les cités où la minorité ne dispose que de la liberté de conscience, celles où les deux cultes peuvent être célébrés publiquement et enfin les villes fondées sur la parité politique et religieuse. Avec comme corollaire, des questions de coexistence confessionnelle d'autant plus nombreuses à régler que le champ des libertés reconnues ou concédées à la minorité est vaste. Avec au cœur des débats, les modalités et la hauteur de la représentation politique de la communauté minoritaire, sa place dans le corps de ville, sa participation éventuelle à l'exécutif citadin, les usages de l'espace urbain lors des processions ou la question du respect du calendrier catholique par les protestants. Autant de problèmes quotidiens, surgis de la coexistence confessionnelle et qui posent des problèmes de fond sur l'essence de l'universitas, l'impact des valeurs inhérentes à la citoyenneté citadine auprès d'une société segmentée en communautés religieuses. Pour répondre à ces questions, les protagonistes des « paix de religion » firent le choix du pragmatisme, d'un traitement technique des problèmes, permettant aux juristes d'acquérir une reconnaissance et une position sociale de premier plan dans les cités comme dans l'État.

Les «paix de religion", promulguées à l'initiative d'hommes d'État pétris de culture juridique, tels les emblématiques Michel de L'Hospital et Lazarus von Schwendi, sont mises en œuvre dans les villes par des praticiens du droit, avertis des réalités de la chose publique et avocats du bien public. Christin souligne cette tension entre centre et périphérie, entre État central et communautés urbaines. Celles-ci crurent à la viabilité des «paix de religion » définies schématiquement par le pouvoir central auquel elles demandèrent d'être le garant des traductions particulières de son innovation politico-religieuse. Christin peut alors remettre en question le lien généralement posé entre divisions religieuses et affaiblissement du pouvoir souverain et souligner que loin de rendre l'État absent ou évanescent, la diversité religieuse et son mode de résolution par la «paix de religion » lui offre la possibilité d'être un acteur impartial, garant des confessions de ses sujets, respecté et légitimé grâce à sa fonction arbitrale. Dans cette perspective, le massacre de la Saint- 
Barthélemy, assumé par Charles IX ne pouvait que ruiner, au moins de manière temporaire, cette position de l'État et du souverain. Il offrait la possibilité aux théologiens et surtout aux prédicateurs-imprécateurs adeptes d'un retour au triptyque "une Foi, une Loi, un Roi » déjà chimérique, de prendre leur revanche sur les hommes de loi et d'envahir à leur tour l'espace politique dans les années 1580-1590. Ce combat des « fidèles papistes » se révéla sans traduction politique durable, aussi, sans céder à la prospective rétrospective justement égratignée par l'auteur, l'on peut créditer les "paix de religion » et leur souvenir d'une certaine influence dans l'échec politique de la Ligue et l'acceptation laborieuse de l'édit de Nantes.

L'essai de Christin, servi par une démarche comparatiste stimulante, la mobilisation de sources aussi diverses que des traités politiques, des délibérations consulaires, des allégories, l'emploi des méthodes de l'anthropologie et de la sociologie historiques emporte l'adhésion et démontre de manière salutaire l'inanité du finalisme brocardé avec vigueur (p. 202-203). Il y a bien eu dans l'Europe médiane un moment des « paix de religion », qui a correspondu en France aux premières années du règne de Charles IX et dont l'échec n'était pas inéluctable. Sans doute, suivrat-on moins facilement l'auteur dans son refus d'envisager que les édits de pacification, imposant à chacun de pratiquer son culte avec réserve n'aient pas favorisé l'intériorisation confessionnelle. N'y a-t-il pas dans cette séparation souhaitée et recherchée entre le politique et le religieux, une voie d'accès, des effets induits en faveur de l'autonomisation de la raison politique cernée de manière convaincante par Christin.

Michel CASSAN

Reuven A. Brenner, Gabrielle Brenner, Spéculation et jeux de hasard. Une histoire de l'homme par le jeu. Trad. de l'anglais par Marie-Andrée LAMONTAGNE. Paris, Presses universitaires de France, 1993. $15 \times$ 21,5, 254 p., bibliogr., index (« Libre-échange »).

L'objectif déclaré, et ambitieux, de l'ouvrage est de raconter une histoire de l'homme par le jeu. En effet, il ne s'agit pas seulement d'écrire une histoire des jeux de hasard, mais encore de comprendre ce qui pousse l'être humain à se livrer à ces derniers. Ce projet amène les auteurs à un second propos qui prend, chemin faisant, une grande importance : réfuter tous les dénigrements qui ont visé cette activité et, à l'aide des statistiques, de l'enquête sociologique, de l'économie, de l'histoire, présenter un portrait du joueur, une analyse du jeu de hasard et de la spéculation qui combattent les idées reçues en la matière. C'est donc bien une histoire d'un trait frappant du caractère humain qui nous est ici présentée.

Six chapitres s'enchaînent de la façon suivante. Le premier déroule un historique des jeux de hasard, et particulièrement de la loterie. Le deuxième explique pourquoi les gens s'adonnent au jeu. Le troisième et le quatrième interrogent le fait que, respectivement, le jeu et la spéculation ont souvent été l'objet de condamnations sévères et tentent de l'expliquer. Le cinquième étudie les moyens mis, de nos jours, 
à la disposition des gouvernements pour réglementer cette activité. Le sixième et dernier chapitre tente une synthèse conclusive et, définissant les notions employées dans l'ouvrage, propose une véritable politique du jeu.

L'origine des jeux de hasard est liée au fait que les peuples avaient régulièrement recours au tirage au sort pour connaître la volonté de Dieu sur un certain nombre de questions. En ces temps-là, le hasard n'est qu'une autre figure de l'ordre divin se révélant aux hommes. Dans les « sorts », il s'agit, jusqu'au XVII e siècle, d'en appeler directement à la providence divine : voilà pourquoi l'Église en interdit l'usage sur des questions triviales. Ainsi, la loterie est condamnée pour encourager l'oisiveté et l'insouciance et pour manquer de respect envers Dieu. Les auteurs montrent, à travers une rapide histoire de la loterie, que cette dernière est tantôt interdite, tantôt utilisée par les États pour se procurer des fonds. Mais, du fait des déficits gouvernementaux, la loterie finit par triompher, comme en témoigne l'exemple du Canada. Pourquoi les loteries sont-elles à présent florissantes?

Pour saisir la complexité des attitudes répertoriées ci-avant, interdiction, condamnation virulente, à l'occasion permissivité, il importe de savoir pourquoi les gens s'adonnent au jeu et quels groupes sociaux jouent plus que d'autres. Les auteurs montrent, en s'appuyant sur des études statistiques de type sociologique, que la population des joueurs de loterie a tendance à être plus âgée, plus pauvre et à avoir plus d'enfants que la population en général. Il y a également ceux dont, accidentellement, les avoirs réels se sont avérés sensiblement moindres que prévu.

Ce même deuxième chapitre réfute, dans un second temps, l'opinion négative que les « arbitres de la vertu » ont des joueurs, présentés volontiers comme instables, trop confiants en leur bonne fortune, prêts à ruiner la vie de leur famille. Il s'agit d'un mythe qui a été forgé par la littérature sur le joueur. Tout d'abord, les joueurs ne sont pas des analphabètes culturels, ils voient plus souvent leurs amis et leur famille et s'engagent davantage dans leur milieu. On ne trouve pas de liens entre le jeu et le crime, entre le jeu et l'instabilité matrimoniale. Les auteurs montrent ensuite que les gagnants ne gaspillent pas leur argent, ne négligent pas leur famille et ne gâchent pas leur vie. Enfin, les joueurs compulsifs ne représentent qu'une infime fraction de l'ensemble des joueurs. Cette image rassurante n'a pas empêché la condamnation du jeu. Pourquoi?

Le troisième chapitre montre que le jeu soulève un débat théologique et philosophique : hasard ou providence? Un monde régi par le hasard est-il tolérable? Le jeu de hasard, en effet, vise à l'enrichissement du joueur sans qu'il ait à fournir les efforts nécessaires par son travail ou à démontrer ses mérites. Cette controverse se mue, au cours de l'histoire, en question sociale : le jeu est assimilé au loisir de la classe ouvrière et semble menacer l'ordre social défendu par les possédants. Associé - faussement - au vice et au crime, il devient " un nid de révolutions ». Les auteurs montrent que, jusqu'à notre époque, les attaques menées contre le jeu reposent sur des faussetés ou des convictions idéologiques suscitées par l'intérêt de classe. Mais, de nos jours, les gens se mettent de plus en plus à penser que c'est la chance, et non uniquement le flair et le travail qui peut décider de la richesse de chacun. D'où le succès de La Roue de la fortune...

Le quatrième chapitre, qui a le même objectif que le précédent, se veut une défense de la spéculation et des marchés à terme. Qu'est-ce qui a amené à confondre ces pratiques : jouer, parier, spéculer, investir? On comprend la confusion entre le 
jeu et la spéculation puisque les joueurs et les spéculateurs visent le même but : s'enrichir rapidement et ce, sans fournir un effort particulier. Mais on aurait tort de penser que la spéculation aurait un effet déstabilisateur sur la société : les auteurs démontrent, par des exemples historiques, que ce serait plutôt le contraire. Le spéculateur est montré comme celui qui peut avoir raison avant et contre la majorité. Il faut alors se garder de confondre spéculation, fraude et crime. De même, l'histoire des assurances, d'abord identifiées au pari et à l'usure - et condamnées de ce fait - démontre que leur action est économiquement bénéfique, voire nécessaire; car, et c'est une thèse fondamentale de l'ouvrage, l'existence de l'assurance, tout comme celle du jeu, aide l'homme à garder espoir en un avenir meilleur. Le joueur assume des risques liés à son rang social tandis que le spéculateur assume des risques liés au commerce : les rêves et les succès du joueur sont autant de témoignages de sa foi en un avenir meilleur, contre un ordre établi qui se sclérose. Lorsque la loi interdit le jeu et la spéculation, elle étouffe ces espoirs et ces ambitions propres à l'humain.

Le cinquième chapitre examine les différentes options qui s'offrent aux gouvernements pour réglementer le jeu. La tendance actuelle est à la légalisation : c'est bien le signe que les dépenses des gouvernements ont brusquement augmenté et qu'il leur faut trouver de nouvelles sources de financement. De nouveaux griefs frappent alors le jeu : les gouvernements, les systèmes de taxation, le crime et les jeux de hasard n'ont-ils pas partie liée? Les auteurs montrent qu'il n'en est rien, en étudiant les conséquences néfastes de la prohibition des loteries. Celle-ci ne conduit qu'à augmenter les impôts ou réduire les dépenses. D'ailleurs sa sincérité est-elle si évidente? Ne cache-t-elle pas parfois des intérêts purement égoïstes? Il n'en demeure pas moins que la confusion règne encore aujourd'hui dans beaucoup d'États à propos des lois sur le jeu. Selon les auteurs, il faudrait en venir à briser le monopole de l'État en la matière. De telles mesures conduiraient à une situation à la fois créatrice d'emplois et susceptible d'encourager l'optimisme et l'espoir chez ceux que la vie a davantage malmenés.

Le sixième chapitre est une conclusion. On aurait tort de croire, et l'histoire racontée dans cet ouvrage le prouve, que l'interdiction du jeu entraînerait sa disparition. Est-il donc en accord avec le bien commun? Les auteurs réaffirment que le jeu ne mine pas l'éthique du travail et qu'il ne fait pas primer l'argent et l'appât du gain sur le souci du bien commun. Mais qu'est-ce que ce «bien commun »? Il consiste essentiellement dans le bonheur, «bon heur" rappellent les auteurs : chance et hasard. On peut alors définir la notion de bien commun par l'existence d'espoir et d'occasions d'avoir de la chance. Mais, si cette définition est juste, il demeure impossible de déterminer quelle quantité de jeu peut être socialement acceptable. Quelles politiques se font-elles néanmoins jour à la lumière de la précédente définition? La légalisation du jeu, affirment les auteurs, peut conduire au développement de tout un nouveau secteur offrant à la fois des possibilités d'emploi et des partenariats d'entreprise.

Il ne s'agissait donc pas tant de livrer des informations - et elles sont nombreuses dans cet ouvrage enrichi d'une abondante bibliographie par chapitre - que de s'élever contre des idées reçues. De même, il s'agit de poser des problèmes complexes, et non de livrer une interprétation seulement économique, psychologique ou sociologique du jeu de hasard, en des termes trop cloisonnés. C'est l'histoire de l'homme et de sa complexité qui est en jeu. Mais cette convocation de dif- 
férentes disciplines - on peut parler d'interdisciplinarité - que pratiquent avec virtuosité les auteurs conduit parfois à un peu de confusion et à quelques redites. Le chapitre de conclusion aidera le lecteur à mettre en rapport les thèses les plus importantes mais trop clairsemées et répétées dans l'ouvrage, ce qui en rend parfois malaisés le suivi et la synthèse.

Jean-Marc RoHRBASSER

Ralph CuDworth, Traité de morale, "Traité concernant la morale éternelle et immuable " et Traité du libre arbitre. Introd., trad., glossaire, bibliogr. et notes par Jean-Louis Breteau. Paris, Presses universitaires de France, 1995. $15 \times 21,5,377$ p., index («Fondements de la politque »).

On doit à Jean-Louis Breteau, angliciste qui a consacré sa thèse à Ralph Cudworth, et au soutien d'Yves-Charles Zarka, responsable de la collection, de disposer en édition moderne de cette traduction. Sans doute s'agit-il avec Cudworth d'un de ces minores dont la cohérence n'est pas la qualité dominante et qui pour cette raison peut-être (est-ce sûr?) n'est pas très connu; mais il s'agit aussi d'un de ces auteurs qui ont eu sur leur époque une influence considérable, par la transmission des textes, des doctrines et des questions. Transmission des textes: son ouvrage principal, l'Authentique Système intellectuel de l'univers a servi de recueil de citations de philosophie grecque, non sans développer aussi une philosophie de la physique analogue à celle dont s'inspire Newton. Transmission des doctrines : la relecture de Platon, de Plotin, de Boèce... lui permettait de prendre à l'égard de l'épicurisme renaissant une position à la fois conciliatrice et critique, qui fit date; l'empirisme britannique n'aurait pas été possible, dans sa spécificité souvent méconnue (à savoir son intérêt pour la raison), sans cette atomisme relu par le platonisme. Transmission des questions : son horreur du conventionalisme radical à la Hobbes, son souci d'une morale transcendante, l'ont amené à chercher un équilibre entre la sensation et l'intellection, le corps et l'esprit, le mécanisme et le finalisme, dont Locke a manifestement médité les enseignements, et qui a fait regretter à Leibniz la redécouverte trop tardive de son texte principal. Les textes posthumes publiés ici, quant à eux, peuvent être considérés comme des éléments de discussion avec Locke : sans preuve décisive, l'interprétation fait actuellement son chemin, d'une lecture directe de ces textes par Locke (cf. Stephen Darwall, The British Moralists and the internal ought, 1640-1740, Cambridge, Cambridge University Press, 1995, p. 172-175), voire inversement d'une composition par Cudworth à la suite d'une lecture de manuscrits lockiens; ce texte serait ainsi une sorte de Nouveaux Essais avant la lettre, qui aide à comprendre ce que l'empirisme et le néoplatonisme ont de parenté dans leur opposition même. Cudworth minor peut-être, mais qui mérite donc plus d'être connu que son collègue de Cambridge, Henry More, dont la correspondance avec Descartes analysée par Koyré notamment, n'a pas toujours la même profondeur. 
L'intérêt des textes publiés et traduits tient à ce qu'ils présentent en une forme infiniment plus ramassée que le monstre qu'est le Système intellectuel, les thèses épistémologiques, cosmologiques et morales. Car le titre du premier Traité ne doit pas tromper: il s'agit beaucoup plus, ici, de théorie de la connaissance que de morale. L'objectif est d'établir les conditions préliminaires d'une théorie de la loi de Nature; et la cosmologie d'abord, les modalités de la connaissance ensuite sont au principe de la morale : l'épicurisme d'abord déforme la saine conception de l'atomisme démocritéen en introduisant le hasard au principe de l'ordre, alors que l'origine mosaïque de l'atomisme (thèse syncrétique plusieurs fois reprise) liait les atomes par des formes spirituelles. Le relativisme de Protagoras, le conventionalisme de Hobbes, l'empirisme de Gassendi - et peut-être des manuscrits de Locke -, le criticisme enfin de Descartes (et de Locke encore) qui prétendent mettre en examen les capacités de connaître avant de commencer à connaître, sont toutes des formes de gnoséologie qui remettent en cause la nécessité des essences et donc l'antériorité des principes moraux.

Appuyé sur le Thééthète, et le livre X des Lois surtout (mais aussi un Aristote et un Descartes platonisés), Cudworth s'oppose à toute mesure humaine du connaissable. La distinction du sensible et de l'intelligible, remise en honneur par Descartes, permet de différencier l'effet de pure réceptivité passive, propre à la sensation, et l'activité mentale de la perception qui exerce sa propre activité en harmonie avec (et non à l'occasion de) la sensation. La sensation, cette «perception assoupie », ne peut être fausse par elle-même puisqu'elle est pur effet; elle participe déjà à l'intellect en ce qu'elle interprète comme qualités secondes certains effets des corpuscules, mais cet « assoupissement » de la sensation l'empêche de sortir du factuel, de l'imparfait, du singulier. S'il y a donc de la norme, du parfait, de l'universel, il ne peut surgir que de l'esprit qui crée de son propre fonds (innéité peut-être, mais pas de la connaissance, seulement de la disposition à construire les concepts), les concepts normatifs, parfaits et universels. Et tout autant que les concepts des choses, l'esprit agissant constitue de son propre fonds les rapports entre les choses (les rapports ne peuvent être que mentaux) d'une part, et les distinctions qui déterminent genre, espèce et singulier dans le concept abstrait d'autre part. La détermination de la chose même que l'on connaît est donc effet de l'esprit, autant que sa relation et son essence. Le critère de la vérité demeure dans la clarté de l'idée intelligible, et non dans le sensible, ni d'ailleurs en Dieu où l'on ne peut voir les idées éternelles. Reste que ces essences éternelles doivent exister en une substance, ce qui prouve l'existence de Dieu, et inversement fonde la connaissance, et surtout la morale.

Au-delà de ces grandes lignes, il faut que le lecteur s'intéresse au détail des analyses qui s'attachent aux débats épistémologiques de l'époque : la question de l'idée abstraite constituée par élimination de qualités concrètes, le statut du triangle qui ne serait ni scalène, ni..., le statut des qualités premières et secondes, l'innéité et la priorité de l'universel en toute analyse de ressemblance, tous débats de l'époque qui ont dans ce texte leur solution néoplatonicienne clairement exposée.

Le deuxième Traité, $D u$ libre arbitre est plus explicitement lié à la morale. Ouvertement dirigé contre Hobbes (Of liberty and necessity), mais aussi contre le prédestinationisme calviniste et les formes contemporaines du stoïcisme, dirigé enfin contre la psychologie scolastique (ou vulgaire) des facultés, il s'appuie sur le fait du jugement de blâme et de louange pour établir qu'il existe des choses « qui 
dépendent de nous ". Mais le plus intéressant est sans doute le plus original : la théorie de l'auto-« référence » de l'âme. Thèse fondamentale, en ce qu'elle intègre l'apport de toute la tradition augustinienne dans une réflexion moderne qui servira d'appui à Locke, et par là Hume, Rousseau, voire Kant. C'est parce que l'âme est divisée en elle-même qu'elle peut faillir, mais aussi se réfléchir, se diriger, ce en quoi réside la véritable liberté d'indifférence, non pas perpétuelle indécision, mais capacité de se maîtriser dans le choix du bien. Le pouvoir sur soi, cultivé par Shaftesbury, commenté par Foucault, trouve ici une de ses études les plus intéressantes. Ici encore, une meilleure connaissance de ce texte posthume (publié pour la première fois en 1838) permet de mesurer à l'aune de cette pensée tâtonnante, la signification des systèmes, parfois plus caricaturaux, qui ont été publiés.

L'édition de J.-L. Breteau est belle et scrupuleuse; pour le second Traité, dont les manuscrits sont disponibles, l'édition est critique en ce qu'elle reprend les variantes les plus significatives. La traduction élégante justifie ses choix par un glossaire et par de nombreuses citations en langue originale données en notes, y compris, ce qui est souvent éclairant, des textes grecs cités par Cudworth et leur traduction actuelle (les partis pris de Cudworth se remarquent ainsi, par exemple dans sa citation de Boèce, p. 131, où l'aristotélisme d'un texte de Boèce devient un platonisme); sans doute renâclera-t-on devant le choix (justifié) de traduire to exert par raviver (exert ideas = raviver des idées), qui a l'inconvénient de transformer l'innéisme dispositionnel de Cudworth en innéisme du contenu, alors que le traducteur a lui-même conscience du danger de confusion (mettre en ouvre pouvait éviter cette connotation ambiguë). La redécouverte des sources, par un travail personnel considérable qui ajoute à celui des éditeurs anglais et allemands, est précieuse. L'introduction et les annotations thématiques sont discrètes - trop sans doute quand on connaît la compétence de l'auteur - mais sans doute liées aux exigences éditoriales; une ample bibliographie permettra d'approfondir le travail. Un texte trop ignoré, scrupuleusement édité, donc, et qui devrait favoriser une meilleure mise en perspective des $\mathrm{XVII}^{\mathrm{e}}$ et XVIII ${ }^{e}$ siècles britanniques.

Jean-Michel VIENNE

Beccaria et la culture juridique des Lumières. Actes du colloque européen de Genève, 25-26 nov. 1994, études historiques éd. et prés. par Michel PoRret. Genève, Droz, 1997. 15,3 × 22, 318 p. (« Travaux d'histoire éthico-politique », vol. 56).

Le bicentenaire du décès de Cesare Beccaria (1738-1794) a été l'occasion d'un colloque international dont on trouvera ici les actes, enrichis de la réédition d'un texte de Jean-Robert Tronchin (1710-1792), procureur général de la République de Genève de 1760 à 1767; et de l'édition d'une traduction partielle et inédite de $D e i$ delitti e delle pene par Maine de Biran. Deux importants ouvrages sur l'œuvre du célèbre marquis avaient déjà été publiés en 1990 . Le premier offre probablement à l'heure actuelle la source d'information la plus précise sur la vie et l'œuvre de Beccaria, tandis que le second tend à apprécier l'influence des idées du marquis dans les 
législations nationales ${ }^{15}$. Les contributions rassemblées par Michel Porret visaient manifestement la complémentarité avec ces deux ouvrages collectifs en mettant l'accent cette fois-ci sur les conditions de réception et d'applications Des délits et des peines en Angleterre, en Italie, dans les pays du Nord (Danemark, Norvège, Suède), en Espagne et dans les possessions habsbourgeoises. La première partie de l'ouvrage comprend en particulier une étude de Cyprian Blamires sur le rôle de Beccaria dans la pensée des réformateurs anglais (William Blackstone, Martin Madan, Samuel Romilly, William Paley, William Eden et Jeremy Bentham) qui met en évidence les divergences entre les juristes anglais sur l'interprétation des thèses de l'Italien et, surtout, l'influence de Beccaria sur la pensée de Bentham. Beccaria est resté célèbre pour s'être opposé aux conditions d'administration de la justice pénale organisées par l'ancien droit. L'appréciation de l'intentionalité du coupable - effectuée par les juges - était une opération trop variable pour être fiable car elle dépendait d'une multitude de facteurs difficiles à déterminer ${ }^{16}$. Beccaria combattit «l'arbitraire » de cette démarche à coup de syllogismes, dont voici l'un des plus connus : «En présence de tout délit, le juge doit former un syllogisme parfait : la majeure doit être la loi générale, la mineure l'acte conforme ou non à la loi, la conclusion étant l'acquittement ou la condamnation ${ }^{17}$. " En ce sens, le marquis italien prenait position contre l'individualisation de la peine qu'allait revendiquer les positivistes du XIx siècle. Évaluant le rapport en définitive assez lâche de la pensée de Montesquieu à celle de Beccaria, Catherine Larrère rappelle très opportunément, et l'étude de Michel Porret sur le cas de la République de Genève le confirme sur de nombreux exemples, que le qualificatif $d^{\prime}$ ' "arbitraire » s'est peu à peu déplacé au xvIII siècle d'une connotation positive exprimant la neutralité du juge à une expression péjorative - présente dans les Delitti - qui ne rendait pas exactement compte du processus parfois très complexe de qualification des crimes. Si la charge polémique de Beccaria résidait dans cette dénonciation du fonctionnement de la justice, elle reposait aussi, comme le montre Pierre Lascoumes, sur le fait que son utilitarisme et son système de valeurs renvoyait à une conception de l'ordre public laïcisé. Plusieurs contributions (Renato Pasto, Mario Sbriccoli) rappellent ainsi la puissance critique et politique des Delitti dans lequel M. Sbriccoli voit aussi un «pamphlet sur l'égalité » (p. 178). L'essentiel de la force polémique des Delitti réside peut-être dans le fait que son auteur a considéré « la question pénale comme le moyen de traiter d'une manière plus générale la réforme de la société et de l'État » (p. 178). Elle explique aussi certainement la diversité de ses lectures et de ses usages dans les pays d'Europe occidentale.

La seconde partie de l'ouvrage est moins directement liée à Beccaria. Elle rassemble une série d'études assez diverses sur les théories et pratiques pénales dont le point commun est d'affirmer que le Siècle des lumières n'était peut-être pas aussi sombre que celui qui était dénoncé dans les Delitti. Ces dossiers particuliers sur la maréchaussée française (Nicole Dyonet), le processus d'incrimination des coupables

15. Sur l'homme et l'œuvre : Cesare Beccaria tra Milano e l'Europa, Milan/Rome/Bari, Cariplo/Laterza, 1990. Sur la postérité de l'œuvre, Cesare Beccaria and modern criminal policy, Milan, Giuffrè ed., 1990.

16. Cesare Beccaria, Des délits et des peines, $1^{\text {re }}$ éd. 1764, Paris, Flammarion, 1991, p. $75-77$.

17. Ibid., p. 67. 
à Genève (M. Porret), la rareté des peines corporelles en Bourgogne (Benoît Garnot) et l'appel croissant des juges à la médecine légale (Vincent Barras, Alessandro Pastore) convergent pour dresser un horizon d'attente dans la culture juridique du siècle de Beccaria. Si la somme des critiques contenus dans les Delitti ne se retrouve pas dans les textes des jurisconsultes traditionnels, la plupart d'entre eux aspiraient déjà à une codification plus nette des catégories d'incriminations, certains étaient réservés même sur l'emploi de la torture au cours de l'instruction. De ce point de vue, les tentatives de réformes de la justice dans les différents pays auraient pu faire l'objet d'une étude spécifique. Peut-être aurait-on pu également relier la fortune de l'ouvrage de Beccaria à la transformation des représentations du criminel et de la criminalité dans la seconde moitié du XviII ${ }^{e}$ siècle. Celle-ci a été étudiée en détail par exemple sur le cas français par Hans-Jürgen Lüsebrink à travers l'analyse croisée de la littérature des Causes célèbres, dont Louis-Dominique Cartouche (1693-1721) et Louis Mandrin (1724-1755) sont les figures de proue, et celle des mémoires proposés aux concours des Académies en cette fin de xviII ${ }^{e}$ siècle ${ }^{18}$. L'analyse de cinquante mémoires rédigés entre 1774 et 1788 portant sur la criminalité et la justice pénale a permis à H.-J. Lüsebrink de constater que si la nature humaine reste évoquée à 27 reprises comme cause des délits, les " abus sociaux » le sont 38 fois, et les « abus juridiques » 43 . On peut lire dans ces résultats l'expression d'une pensée liée à la critique de la Monarchie absolue bien sûr, mais aussi celle d'une rhétorique éphémère, qui va se dissoudre en France dans l'exercice du pouvoir révolutionnaire. Or il nous semble que c'est bien l'une des caractéristiques, sinon de la culture juridique, du moins d'une certaine pensée juridique des Lumières, que d'avoir cru pouvoir combattre la criminalité avec de bonnes lois. Denis Diderot résuma parfaitement l'esprit de cet idéalisme juridique incarné par Beccaria : «Si les lois sont. bonnes, les mœurs sont bonnes; si les lois sont mauvaises, les mœurs sont mauvaises ${ }^{19}$. » Notons enfin que la dimension médico-légale de la culture juridique du $\mathrm{XVIII}^{\mathrm{e}}$ siècle a fait l'objet de nouvelles études présentées à un autre colloque genevois, en juin 1997. La publication des actes permettra certainement de mieux saisir le rôle pratique de la médecine légale dans ce vaste mouvement de réformes pour lequel Beccaria sert incontestablement d'éponyme.

Marc RenNeville

Jean-Michel BeRTHELOT, Les Vertus de l'incertitude. Le travail de l'analyse dans les sciences sociales. Paris, Presses universitaires de France, 1996. 13,5 $\times 21,5$, 272 p., index (« Sociologie d'aujourd'hui »).

Jean-Michel Berthelot, sociologue, nous convie à travers son dernier livre à un exercice de réflexion sur les sciences sociales. L'ouvrage, solidement charpenté et

18. Hans-Jürgen LƯsEBRINK, «Les Représentations sociales de la criminalité en France au XVIII ${ }^{e}$ siècle », doctorat de $3^{e}$ cycle, Paris, École des hautes études en sciences sociales, 1983, p. 213. Une version remaniée de cette thèse est parue : Kriminalität und Literatur in Frankreich des 18. Jahrhunderts., Munich/Vienne, R. Oldenbourg, 1983.

19. Denis Diderot, Supplément au voyage de Bougainvillée, $1^{\text {re }}$ éd. 1772, Paris, Flammarion, 1993, p. 178. 
bien argumenté, s'organise en plusieurs temps. Jouant de la focale de son objectif, l'auteur part du cadrage le plus large (l'espace des sciences sociales et de leurs schèmes d'intelligibilité) pour aller vers le plan le plus serré (la discipline en son fonctionnement spécifique). Le projet n'étant pas prescriptif mais analytique, il ne s'agit donc pas de proposer une épistémologie normative, mais de tenter d'analyser ces sciences telles qu'elles se manifestent dans leurs multiples productions passées et présentes.

Tout d'abord, chaque recherche en sciences sociales découpe un objet spécifique (un ordre de problème, un type de phénomène...) dans la complexité des réalités sociales. Et, à chaque moment de sa démarche, c'est « l'anticipation d'une structure interprétative » (p. 39) qui guide le chercheur. Berthelot distingue donc six schèmes d'intelligibilité qu'il présente comme des « structures logiques transdisciplinaires et transhistoriques» (p. 84) : les schèmes causal, fonctionnel, structural, herméneutique, actanciel et dialectique. À propos du même "phénomène ", des chercheurs peuvent mettre en œuvre des schèmes d'intelligibilité différents. Ainsi, un fait social tel que le suicide peut-il être étudié dans une perspective causale (en établissant des corrélations statistiques) ou dans une perspective actancielle (en s'attachant à reconstruire des intentionnalités d'acteurs).

Puis, des schèmes d'intelligibilité, on passe aux disciplines. Malgré la transversalité des grands langages d'interprétation, c'est toujours à travers le filtre disciplinaire que se donnent à voir (à lire) les savoirs. L'auteur essaie de sortir de l'antagonisme entre les conceptions externalistes et internalistes des savoirs disciplinaires : une discipline constitue indissociablement un « espace social de légitimation des savoirs » au sein duquel les chercheurs - souvent en conflits ou en luttes - s'organisent en cénacles, en clans, en équipes, en écoles ou en courants, avec leurs revues, leurs collections éditoriales, leurs territoires institutionnels, etc., et un « espace logique de construction d'argumentations » (p. 100) qui est irréductible - quoiqu'en disent les incessantes rumeurs professionnelles sur les « coups " et les « stratégies » des uns et des autres - à la première dimension. S'il serait totalement naïf de nier les déterminations sociales et institutionnelles à travers lesquelels se sont engendrées les productions scientifiques, il serait tout aussi faux de ne comprendre ces dernières que comme de simples effets des premières (p. 123). Les luttes, les rapports de force, les stratégies ou les intérêts..., repérables dans des sphères d'activité extrêmement variées, ne peuvent rendre compte à eux seuls de la logique spécifique des activités scientifiques.

Berthelot ordonne les différentes sciences sociales à partir de deux axes. Le premier oppose un "pôle idéographique", privilégiant la description, à un "pôle nomologique ", prônant la mise en modèle. L'auteur précise bien que si l'on retrouve l'anthropologie et l'économie aux deux extrêmes (la sociologie étant plus près de l'économie et l'histoire se rapprochant davantage de l'anthropologie), une des caractéristiques des sciences sociales réside dans le fait que chaque discipline engendre des travaux couvrant toujours plus ou moins tout l'éventail des possibles. Le second axe de différenciation oppose les disciplines qui circonscrivent très précisément leur champ d'investigation (linguistique, économie, etc.) à celles qui, comme l'histoire, la sociologie ou l'anthropologie n'ont jamais établi de limites à leur enquête (autonomisation vs globalisation). 
Comparant deux livres, l'un d'un sociologue et l'autre d'un historien, Berthelot exemplifie les différences disciplinaires. L'ouvrage d'histoire se caractérise par un «tissage d'événements singuliers et de commentaires théoriques », l'essentiel du texte ne résidant pas dans "l'enchaînement des propositions ". En revanche, dans l'ouvrage de sociologie, « il ne s'agit plus là d'étayer rigoureusement chaque proposition d'un substrat empirique, mais de les insérer dans un ordre théorique, où la cohérence démonstrative vaut pour preuve » (p. 134). L'auteur de ce compte rendu, sociologue de métier, ne peut s'empêcher ici de sortir de sa réserve descriptive et de la posture qui sied à l'épistémologie analytique pour émettre un commentaire normatif : l'exemple d'ouvrage sociologique pris par Berthelot donne assez spontanément envie à tout sociologue animé par l'esprit d'enquête de se déclarer historien. En effet, on est en droit de se demander si, « davantage sensible à l'agencement des raisons et à une théorisation du fonctionnement actuel des sociétés qu'à une mise à l'épreuve empirique » (p. 139), le sociologue ne sort tout simplement pas de son rôle (en se faisant herméneute sauvage ou logicien formaliste) et si l'historien qui «ne s'autorise de généralisations que sous le contrôle minutieux d'une multiplication des faits et des références » n'est finalement pas à meilleure distance du théoricisme pour faire la science des faits sociaux. Les sociologues ne gagneraientils pas, sans perdre leurs ambitions théoriques, à travailler avec une âme d'historien? Bien sûr, l'exemple pris par l'auteur n'est absolument pas en cause ici, car les productions sociologiques se caractérisent effectivement largement par cette « structuration rationnelle des faits [...] sous faible contrainte » (p. 140). Mais l'épistémologie analytique proposée, éclairant les axes différenciateurs, les lignes de tension ou de fracture entre les disciplines, doit justement permettre, dans une perspective cette fois-ci plus normative, d'envisager plus consciemment une politique (sociologique, historienne, etc.) de la recherche.

Berthelot entre ensuite au cour d'une discipline pour en inspecter la logique interne. C'est l'exemple de la sociologie qu'il propose alors de développer en mettant au jour les différents styles d'analyse pratiqués : sociographie, sociologie expérimentale (animé par le souci de «mise à l'épreuve empirique »), sociologie interprétative (refusant « toute investigation objectivante » et flirtant avec la littérature), sociologie critique (dont le travail de P. Bourdieu et J.-C. Passeron, La Reproduction, représenterait l'idéal-type) et sociologie analytique (« entreprise de réflexivité interne à la sociologie »).

Il conclut, enfin, sur la question des liens entre les sciences sociales et l'action, en faisant alors clairement ressortir l'acquis incontestable des sciences sociales, à savoir « la reconnaissance de l'incomplétude et l'appréciation du risque d'incertitude immanents aux savoirs scientifiques " face à « tous les discours totalitaires d'affirmation péremptoire d'une vérité révérée » et appelle de ses vœux « l'ouverture à la société entière d'une discussion argumentative, dont la science fait quotidiennement l'expérience dans son travail de validation, mais que seul l'ensemble des concernés peut entreprendre, dès lors qu'il ne s'agit plus seulement du vrai, mais du bien » (p. 247).

On pourrait adresser une série de remarques critiques à l'auteur : l'absence de dialogue ou de confrontation directe avec les réflexions épistémologiques, parfois 
congruentes mais parfois aussi différentes, de Jean-Claude Passeron ${ }^{20}$; l'utilisation d'un langage formel qui ne rajoute rien à ce que dit - bien - l'auteur dans la «langue naturelle " (tout lecteur peut faire l'expérience consistant à lire d'abord sans les formalisations, puis avec les formalisations, les mêmes passages du livre et constater alors la faible plus-value sémantique ainsi dégagée); la tendance paradoxale, par souci d'attitude épistémologique non normative, à légitimer objectivement les entreprises de connaissance les plus rétives à ce qui nous semble constituer des exigences minimales en sciences sociales. Ainsi l'auteur évoque-t-il le «style interprétatif » en sociologie de la façon suivante : «Souvent rejeté aux marges d'une scientificité tatillonne, prêtant le flanc aux critiques par ses faciles glissements vers une élaboration littéraire et son mépris explicite de toute logique de la preuve, menacé en permanence par le cercle du sens, ce style tire néanmoins sa force et sa légitimité des dimensions secrètes auxquelles il contraint à l'attention » (p. 206), et à entrer en fin de compte - normativement - dans les luttes pour la définition légitime des sciences sociales.

Mais la curiosité intellectuelle l'emporte, qui pousse plutôt à se demander ce que le même objet de réflexion pourrait susciter comme démarche de la part d'un historien. Ce dernier considérerait sans doute comme une trop grande audace le fait de supposer la transdisciplinarité et, surtout, la transhistoricité des schèmes d'intelligibilité. Il insisterait certainement davantage sur les discontinuités, les variations et la spécificité de certains contextes historiques, là où le sociologue peut être travaillé par la pulsion, socialement constituée, de la généralisation hâtive de son propos. En un temps où anthropologie, histoire et sociologie, pour ne citer que les disciplines les plus proches, nouent des liens sur des bases moins hégémoniques que par le passé, le livre de Berthelot nous semble particulièrement utile pour éclairer les différences et les spécificités, faire émerger les points communs et aider ainsi les chercheurs des différentes disciplines à s'autotransformer dans un processus de confrontation mutuelle.

Bernard LAHIRE

Denys DE BÉchillon, Qu'est-ce qu'une règle de droit? Paris, Odile Jacob, 1997. $15,5 \times 24,303$ p., index.

La question à laquelle le livre de Denys de Bechillon, chargé de recherche au centre de philosophie du droit de l'université de Paris II, cherche à apporter une réponse est des plus classiques et des plus centrales dans le domaine de la philosophie juridique. On en connaît les difficultés principales : sur le terrain de la normativité, il faut préciser, voire fixer les définitions en rendant compte de manière

20. Jean-Claude PASSERON, Le Raisonnement sociologique. L'espace non poppérien du raisonnement naturel, Paris, Nathan, 1991. 
satisfaisante de la triple dimension descriptive, prescriptive et légitimatrice du droit. Sur le terrain de la définition de la juridicité de la norme, c'est le couple Droit-État qui constitue le nœud gordien.

L'ouvrage se donne pour ambition de clarifier cet écheveau et d'apporter sa contribution à l'océan des thèses sur le sujet, en opérant une double réduction : du droit à la règle de droit et de la règle de droit à la règle étatique de droit. D'après l'auteur, la science est à ce prix.

Cette réduction, justifiée par la méthode constructiviste adoptée laisse donc volontairement de côté le droit comme fait social, en particulier l'étude des institutions qui le produisent et le pratiquent, des discours qui cherchent à le fonder. La perspective choisie est "normativiste » et se réclame explicitement de Kelsen. Les procédures concrètes, sociologiques, anthropologiques et historiques de la genèse de la norme, son inscription dans la société, les conditions requises pour que soit reconnue sa valeur normative ne seront pas examinées : «[...] la norme est "une signification", pas une "chose". Elle constitue un "outil d'une texture purement psychiques" » (p. 166).

La règle de droit est donc une norme. L'auteur écarte toute perspective jusnaturaliste classique ou moderne, plaide pour la distinction la plus ferme de l'ordre descriptif et de l'ordre prescriptif, du fait et de la valeur. De la sorte, «suivant une ligne d'inspiration plutôt kelsénienne, hasardons que la norme pourrait se définir comme la signification d'une proposition, indiquant aux hommes ou aux institutions un modèle auquel conformer leur conduite, impérativement » (p. 165-166).

Comme signification d'une proposition, la normativité de la norme ne provient pas de l'énoncé lui-même, mais de l'autorité qui le prononce : « [...] la question de savoir qui parle et d'où il parle influence donc la manière dont je reçois son propos. C'est elle avant tout qui conditionne les propriétés performatives de l'énoncé [...] c'est avant tout le statut du locuteur qui confère son autorité à un énoncé » (p. 168).

Surtout, la norme dictant un devoir-être est nécessairement impérative. L'habilitation, la permission, l'autorisation et la définition sont certes parmi les critères qui permettent de classer les normes, mais cela ne suffit pas, les normes ont pour objet de «fabriquer de l'interdiction; prescrire, commander, obliger» (p. 177), puisque la nature et ses lois, en elles-mêmes, ne prescrivent rien, et que comme le montre la «belle histoire » qu'est le mythe du Père de la horde primitive, "l'humanité se construit contre » (p. 201). Plus précisément, « ce contre quoi il y a à lutter en tout premier lieu pour assurer la possibilité d'une vie sociale, c'est un désir. Et pas n'importe quel désir, mais celui, sauvage et inextinguible, de me prendre pour le père de la horde, [...] la condition de la civilisation, c'est l'existence d'impératifs catégoriques » (p. 204).

Conscient de la fragilité scientifique de la parabole freudienne, Béchillon l'adopte cependant (« Peu importe en effet que l'histoire du père de la horde soit "vraie" ou non - mettons même qu'elle soit "fausse", et qu'elle ne conte strictissimo sensu qu'un mythe. La narration freudienne n'en apparaît que plus géniale. Car il faut du génie pour forger un mythe digne de ce nom ", p. 201.) et ce faisant, déroute quelque peu le lecteur qui tenait pour parfaitement distincts l'ordre descriptif - scientifique - auquel le livre se rattache et l'ordre prescriptif auquel appartiennent les considérations sur l'utilité de l'impératif et sa légitimation mythique. 
Ce retour de l'impératif au cœur de la définition de la norme ne doit pourtant pas être compris comme une opposition au droit postmoderne, le soft law, au contraire. " À la dureté archaïque d'un Droit "moderne" en fin de parcours, succéderait la fluidité adoucie d'un Droit "post-moderne" plus adulte, plus soft, plus contractuel et assez nettement affranchi de sa dimension autoritaire » (p. 207). La conciliation est rendue possible en distinguant les modes de productions de la norme, négociés, dialogiques, adaptables, et la teneur de la norme, impérative.

Une définition de la norme une fois obtenue, il reste à préciser en quoi dans le vaste champ de la normativité, on peut distinguer une norme particulière, la règle de droit.

Y a-t-il du droit en dehors de l'État? Béchillon, citant Hans Kelsen, opte pour une définition juridique de l'État, parce qu' « il est impossible [de] donner [de l'État] une définition non juridique » (p. 101). Il y voit en premier lieu « la personnification de l'Ordre juridique » (Michel Troper, p. 101), un «corps abstrait» (Ernst Kantorowicz, p. 103), ce qui lui permet d'affirmer que «c'est la raison pour laquelle la démocratie suppose l'État: le lieu du pouvoir y reste indisponible et simplement occupé par un représentant (du peuple, de la nation, de la volonté générale [...]) » (p. 103).

En second lieu, «l'État suppose [...] une puissance singulière » (p. 104), l'exercice de sa souveraineté, illimité, sauf par la souveraineté des autres Etats, qui fonde la puissance d'action ou de gouvernement du territoire et de la population. La Nation, comme État, et le Droit ne font qu'un. C'est ce droit étatique officiel que Béchillon adopte comme objet, au terme d'un raisonnement en deux temps.

1. Certes, il existe des droits non étatiques. L'examen des cultures non occidentales nous fait découvrir des formes de normativités qui ne font pas intervenir les mêmes paradigmes, les mêmes représentations de la normativité, du temps, du corps, de la causalité... Du droit différent, donc, mais du droit quand même, pour ne pas répéter les erreurs de l'anthropologie coloniale considérant l'absence de droit comme un symptôme de primitivisme. Un tel choix ne peut être considéré comme anthropocentrique parce qu'il n'y a pas de relation entre la question posée par la science (est-ce du droit?) et la distinction, au sein de la normativité, de l'élément séparé droit en Occident : "Ne confondons pas une définition scientifique avec l'observation scientifique d'un phénomène de "définition" sociale " (p. 135). Le droit peut n'exister qu'en Occident, les concepts de la théorie du droit, pure, exclusivement descriptive, sont partout pertinents, si la construction conceptuelle trouve à y gagner. C'est ce que pense Béchillon qui choisi d'appeler la normativité non occidentale «Droit» pour faire sa place aux leçons de l'anthropologie du droit.

2. Béchillon préfère pourtant se concentrer sur le droit étatique parce que «c'est [lui] qui constitue la forme moderne de la juridicité " (p. 143). Choix restrictif, commandé par l'intérêt du point d'observation, qui évacue sciemment le "droit officieux » p. 146, de même que les représentations profanes de la règle de droit (p. 151), celle des simples individus, ou la pratique concrète de l'État (p. 152), pour privilégier le noyau du droit officiel dont la fonction est déterminante. Dès lors, il est possible de définir la norme juridique du droit officiel de l'État comme norme investie comme telle par l'État. C'est l'affiliation au système juridique qu'est l'État qui fait la juridicité de la norme. 
Posant dès les premières lignes (« Je ne sais pas ce qu'est le "juste”, ni même la justice. J'aimerais bien pourtant mais je n'arrive à rien faire qui ressemblerait à de la science avec ces mots-là [...] je crois bien que le Droit est froid, désespérément froid », p. 9) la distinction entre description et prescription, ou pour le dire autrement la neutralité axiologique de la doctrine, la pureté de la théorie, Béchillon conclut en retrouvant son point de départ. Démarche qui a son prix, puisqu'il aura fallu écarter systématiquement, et parfois au nom du but à atteindre, tout ce qui aurait été susceptible de «réchauffer »le problème, voir de brûler la théorie... à savoir la pratique de la norme et son histoire, le mystère de son élaboration concrète, les procédés de légitimation qui l'accompagnent. La question du droit juste surgit d'ailleurs, au terme de l'ouvrage, à l'occasion d'une réflexion sur le droit nazi. Pour l'auteur, dans l'hypothèse où l'État nazi est un État, le droit nazi est du droit, et la critique doit chercher ses armes dans la morale et dans le droit international. Où la théorie, dans sa pureté, se fait prescriptive, puisqu'elle interdit de juger de la justice des lois en vigueur au nom du droit...

D'où un soupçon : si le caractère prescriptif de la norme naît de l'autorité de celui qui l'énonce, suffit-il que le juriste refuse toute prétention à prescrire pour que ce soit le cas? Est-il prouvé sociologiquement que la doctrine, fût-ce sous la forme de la science pure du droit, n'intervient en rien dans l'élaboration et la légitimation du droit? Si tel n'est pas le cas, dans l'affrontement concret entre plusieurs formes de normativités, le droit froid, souple et ferme, occidental et mondial, contractuel et étatique reçoit là le soutien incontestable qui le légitime en le constituant comme objet de science...

Mikhail XIFARAS

Michel FouCaUlt, «Il faut défendre la société ». Cours au Collège de France (19751976), éd. établie dans le cadre de l'Association pour le centre Michel-Foucault, sous la dir. de François Ewald et Alessandro Fontana, par Mauro Bertani et Alessandro Fontana. Paris, Seuil/Gallimard, 1997. 15,3 × 24, XI-291 p., index (« Hautes Études »).

Il y avait l'œuvre exotérique, celle qui, de l'Histoire de la folie à l'Histoire de la sexualité, bouleversa le champ historico-philosophique. Il y eut ensuite le grand rassemblement, protéiforme et punctiforme, des Dits et écrits (Paris, Gallimard, 1994, 4 vol.), des interventions plus ou moins urgentes, plus ou moins immédiatement militantes. Et voici que paraît le premier volume de l'œuvre ésotérique, celle que professa Foucault, au Collège de France, de 1971 à 1984. C'est le texte oral du cours de 1976 qui se trouve ici soigneusement transcrit par Mauro Bertani et Alessandro Fontana, complété par des notes qui en précisent les références, par un «Résumé du cours » extrait de l'Annuaire du Collège de France, enfin, par une brève et éclairante mise en perspective et de fort bienvenus index.

Contrairement à ce qui se produit souvent dans des cas de ce genre, le grand soin apporté par les éditeurs à cette publication n'est pas disproportionné au regard du 
texte même. Il ne s'agit pas d'un fond de tiroir destiné aux fidèles parmi les fidèles, mais d'un véritable « livre », admirablement composé, dont l'essentiel ne redouble pas le propos des ouvrages « officiels » entre lesquels il s'insère - Surveiller et punir (Paris, Gallimard, février 1975) et La Volonté de savoir (Paris, Gallimard, octobre 1976) ${ }^{21}$. Ce n'en est pas l'ombre ou l'ébauche, c'est l'élaboration du problème suivant : dès lors que la circulation du pouvoir ne doit pas être pensée en termes de cession contractuelle (d'aliénation volontaire), «ne faut-il pas l'analyser d'abord et avant tout en termes de combat, d'affrontement ou de guerre » (p. 16)? Et poser ce problème, c'était éprouver la pertinence d'un modèle agonistique (gauchiste?) auquel Surveiller et punir avait, en effet, recouru (p. 31 et 315) pour en finir avec celui du pacte et de la souveraineté ${ }^{22}$.

Il s'agit donc, très philosophiquement, de transformer une solution en une difficulté. Comme toujours, au lieu de demander brutalement : « Cette proposition est-

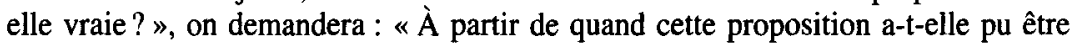
formulée et susceptible de vérité et de fausseté? » Soit, en l'occurrence : «Comment, depuis quand et pourquoi a-t-on commencé à percevoir ou à imaginer que c'est la guerre qui fonctionne sous et dans les relations de pouvoir » (p. 41)?

Pour répondre à cette question, Foucault, soucieux comme à l'habitude d'écarter les trop attendus parrainages du grand Musée philosophique, révoque Machiavel et Hobbes - le premier parce qu'il restait du côté du pouvoir et du prince (p. 145 et 150 ; Volonté de savoir, p. 128), le second parce que, loin de faire de la guerre la matrice du politique, il lui substitue l'état de guerre qui est tout autre chose, à savoir précisément l'éviction de la guerre réelle, empirique, qui se trouve au cœur des polémiques anglaises de l'époque (p. $78 \mathrm{sq}$.). C'est donc plutôt de ce dernier côté qu'il faut se tourner, là où le problème se pose, non dans les termes d'une genèse hypothétique de la souveraineté, mais dans ceux d'une histoire effective des relations de pouvoir, d'un processus régional dont la guerre perpétuelle est le chiffre qui requiert un nouveau savoir, celui de l'historien partisan destiné à infléchir lesdits rapports de pouvoir qu'il s'efforce de penser — un savoir qui donc, pour la première fois depuis Platon, n'est plus de l'ordre de l'ordre et de la paix (p. 154).

Éprouver la pertinence du modèle agonistique, ce sera alors en reconstituer la généalogie par l'étude d'un corpus ailleurs absent et à l'étude duquel est consacré l'essentiel de ce cours, à savoir celui que constituent ces histoires de France où l'on tente de comprendre comment l'origine belliqueuse des invasions barbares s'est trouvée pervertie de telle sorte que les vaincus l'ont finalement, et perfidement, emporté sur leurs vainqueurs. Trois noms, ou plutôt trois ouvrages principaux, balisent alors l'analyse : François Hotman, Franco-Gallia, 1573; Henri de Boulainvilliers, L'État de la France..., 1727; Emmanuel Joseph Sieyès, Qu'est-ce que le Tiers-État?, 1789. Au premier, il revient d'avoir reconduit la question de la légiti-

21. Ce cours, publié en italien dès 1990 (Difendere la società, Florence, Ponte alle grazie), avait d'ailleurs déjà retenu l'attention; cf., par ex., Michel SENELLART, « M. Foucault : "Gouvernementalité" et raison d'État », La Pensée politique, 1, mai 1993, p. 284.

22. Voir p. 195-196, 224, 310, 314. Comment récuser le paradigme contractuel, c'est là un problème qui, en France, se posait à tous : voir la préface de Gilles DeLEuze à Instincts et institutions, Paris, Hachette, 1953, ou l'analyse faite par Louis Althusser, Montesquieu, la politique et l'histoire, Paris, Presses universitaires de France, 1959. 
mité à celle de l'invasion autour de laquelle vont désormais se nouer tous les débats juridico-politiques (p. 104). Au second, qui occupe véritablement le centre de ce travail, il faut attribuer le mérite - mais tout le problème est de savoir si c'est bien d'un mérite qu'il s'agit - d'avoir vraiment pensé l'histoire en tant que calcul des forces, soit un processus qui se définit sans reste par une guerre des « races » ou des « nations », entendues indépendamment de l'État comme communautés d'individus unis par des mœurs, des usages, voire une loi particulière (p. 117 et 143). Au troisième, il faudrait plutôt reprocher d'avoir « embourgeoisé » le discours historique en le recentrant sur l'État dont la nation n'est plus que la figure en pointillé (p. 200); corrélativement, la guerre pour la domination, le conflit de type militaire, n'apparait plus comme la vérité de l'histoire, mais comme l'expression de simples crises exceptionnelles car ce qui conduit désormais le cours des choses, c'est une lutte civile qui a pour enjeu l'État comme figure présente de l'universel - « Le présent, ce n'est plus le moment de l'oubli ${ }^{23} »$. Foucault peut alors relire, pour les renvoyer dos à dos, François Dominique de Montlosier et Augustin Thierry.

À partir de là, il s'efforce de concevoir l'émergence du racisme d'État moderne en référence à celle d'un nouveau mode d'exercice du pouvoir, la biopolitique, qui vient s'articuler à celui de la discipline, analysé en 1975, et qui occupera la dernière partie de La Volonté de savoir : s'il faut opposer la norme à la loi, il faut encore distinguer la norme disciplinaire, qui dresse le corps individuel comme foyer de forces utiles, d'avec la norme régulatrice qui institue les grands équilibres vitaux d'une population définie en termes statistiques. Le racisme moderne apparaît là, au croisement de la vie comme politique et de la guerre comme réalité du pouvoir. La guerre, c'est alors l'extermination de l'ennemi dégénéré par un État qui a en charge l'intégrité de la population entendue comme race biologique. Et le racisme, c'est ce qui autorise le droit de donner la mort là où le pouvoir $\mathrm{a}$, au contraire, pour vocation de majorer la vie, de «faire vivre et laisser mourir ». C'est pourquoi « il n'y a guère de fonctionnement moderne de l'État qui, à un certain moment, à une certaine limite, et dans de certaines conditions, ne passe par le racisme» (p. 227). Y compris l'État socialiste pour qui l'adversaire aurait pourtant dû être de classe, et non de race. Y compris encore - faut-il le préciser? - l'État français d'aujourd'hui ${ }^{24}$.

Ces analyses, pour éblouissantes qu'elles soient, peuvent laisser, à certains égards, dubitatif. Quant à leur pertinence historique d'abord et il faudrait, par exemple, se demander si les discours historiens étudiés par Foucault ne sont pas plus contractualistes qu'il ne veut bien le dire : n'est-ce pas, au fond, d'un pacte historique qu'il s'agit chez Boulainvilliers ou chez Jean-Baptiste Dubos, de ce que Montesquieu appelle, dans L'Esprit des lois (liv. XXX), un « règlement général » - L'Esprit des lois sur lequel Foucault fait d'ailleurs l'impasse aussi radicalement que dans Surveiller et punir à propos du droit pénal, et ce alors même qu'il en est si souvent bien proche ${ }^{25}$ ? Mais, d'autre part, il est frappant de constater que ce para-

23. p. 203. Voir Pierre Macherey, «Une nouvelle problématique du droit : Sieyès », Futur antérieur, 4, printemps 1991.

24. Voir Étienne Balibar, La Crainte des masses, Paris, Galilée, 1997, p. 323 sq.

25. Cela reste à montrer. Quant à la question précise du droit pénal, voir Catherine LARRÈRE, "Droit de punir et qualification des crimes de Montesquieu à Beccaria », in Beccaria et la culture juridique des Lumières. Actes du colloque européen de Genève, 25-26 nov. 1994, études historiques éd. et prés. par Michel Porret, Genève, Droz, 1997. 
digme guerrier dont Foucault relativise ici généalogiquement la pertinence, il ne parvient pas encore à s'en affranchir : s'il s'agit de savoir en quoi des concepts militaires comme ceux de tactique ou de stratégie sont opératoires (p. 40), il faut bien reconnaître que c'est précisément en tant que " tactique discursive » pourvue d'une grande «polyvalence stratégique » qu'est analysé le nouveau savoir historique (p. 67 et 169), de sorte que le serpent se mord ici la queue. Aussi bien, quoique le retournement de la formule de Clausewitz — « La guerre n'est que la continuation de la politique par d'autres moyens » —, qui était opéré brutalement en 1975 (Surveiller et punir, p. 170) et qui sert ici de point de départ (p. 16), se voie bien plus prudemment reformulé en 1976 (La Volonté de savoir, p. 123), la terminologie ne semble guère varier d'un ouvrage à l'autre, le pouvoir y étant toujours décrit en termes de stratégie et de tactique. L'hypothèse militaire s'avère ainsi plus résistante que «l'hypothèse répressive » (p. 17-18) et l'on peut raisonnablement supposer que cette difficulté ne sera pas étrangère aux importants déplacements effectués dans les derniers volumes de l'Histoire de la sexualité. La parution des cours ultérieurs devrait éclairer cela.

Bertrand BINOCHE

Habermas, la raison, la critique. Sous la dir. de Christian Bouchindhomme et Rainer Rochlitz. Paris, Cerf. 1996. 13,5 × 21,5, 240 p. (« Procope »).

Jürgen Habermas, Droit et morale. Tanner lectures (1986). Trad. de l'allemand par Christian Bouchindhomme et Rainer Rochlmz. Paris, Seuil, 1997. $18 \times 25,7$, 93 p. («Traces écrites»).

Le premier ouvrage, qui contient huit études d'auteurs français et étrangers sur Jürgen Habermas, contribue à corriger un déséquilibre de l'édition française, où l'abondance relative des traductions des œuvres de ce philosophe s'accompagne de la rareté des études qui lui sont consacrées. La caractéristique de ce recueil, qui explique son grand intérêt, est de présenter un certain nombre d'approches souvent distanciées, voire critiques, des conclusions habermassiennes tout en évitant de les soumettre à une contestation globale ou de leur opposer des présuppositions radicalement étrangères aux siennes. On distinguera trois ensembles dans ces recherches.

Un premier groupe d'articles s'attache à rendre raison de quelques aspects obscurs de l'évolution habermassienne récente. Le travail de Louis Quéré montre ainsi que derrière la multitude intimidante des références aux sciences humaines auxquelles recourt la Théorie de l'agir communicationnel, le propos habermassien développe une intention claire et simple au fond, susceptible d'intéresser les praticiens des sciences sociales les plus soucieux de réfléchir sur les fondements de leurs démarches : l'intention d'élaborer une anthropologie nouvelle axée sur le thème de la communication linguistique et des formes concrètes de l'intersubjectivité, qui doit se substituer aux paradigmes classiques centrés sur la conscience et l'individu, avec lesquels les sciences humaines hésitent parfois à rompre. De son côté, Rainer 
Rochlitz veut justifier la place de plus en plus prépondérante que Habermas a reconnu à la théorie juridique, alors que jusque dans les années quatre-vingts, sa position dans ce domaine semblait en somme se situer dans l'horizon de la critique marxiste du droit. Cette orientation est liée, montre l'auteur, à une interrogation sur la manière dont la rationalité pratique peut acquérir une efficacité dans les conditions imposées par le monde contemporain. L'investissement du terrain juridique n'a donc pas pour origine une capitulation devant la réalité existante et un reniement du projet critique, mais la conviction que les principes universalistes et égalitaristes de l'éthique ne deviennent effectifs que dans la mesure où ils s'incarnent dans les pratiques d'une démocratie radicale.

Le second groupe d'études de ce recueil est consacré au thème de la raison. Comme on sait, l'ambition philosophique dominante de Habermas consiste à édifier une théorie de la raison, du moins à montrer à quelles conditions on peut effectuer en même temps une reprise contemporaine du thème hégélien de la raison dans l'histoire et de l'exigence kantienne d'une autonomie de la raison pratique. Les articles de Christian Bouchindhomme, Johann Arnason, Jean-Marc Ferry et Plinio Walder Prado, tout en reconnaissant la portée d'une telle tentative, émettent, chacun dans un style différent, des doutes quant à son succès. Le premier met en cause un rapport aux sciences positives qui manque peut-être de clarté critique. Le deuxième s'interroge sur la conception habermassienne de la rationalité, qui reste sans doute trop unilatéralement pensée en fonction de l'opposition de l'instrumental et du communicationnel et de la mise en valeur de ce dernier. L'auteur, en s'appuyant sur Charles Taylor et Maurice Merleau-Ponty, esquisse une définition de la raison qui se voudrait plus large et mieux fondée que celle à laquelle l'auteur de la Théorie de l'agir communicationnel parvient. Ferry, pour sa part, revient sur l'importante polémique entre Habermas et Karl Otto Apel à propos du statut des énoncés philosophiques portant sur la raison: sont-ils à concevoir, comme dans la Théorie de l'agir, à la manière d'explicitations de certaines compétences données empiriquement dans l'histoire et dans les pratiques ordinaires, ou bien, comme chez Apel, sur le modèle des énoncés transcendantaux obtenus au cours d'une démarche de fondation ultime? L'auteur refuse les deux termes de cette alternative et propose une solution personnelle, d'inspiration fichtéenne, qui mérite la discussion. Enfin, Walder Prado rappelle les tenants et les aboutissants du différend entre Jean-François Lyotard et Habermas. En refusant la notion de postmodernité, ce dernier n'a certes pas tort, selon lui, de rejeter l'idée selon laquelle l'époque contemporaine serait marquée par l'éclatement irrémédiable des discours et des pratiques, obligeant à tenir pour caduque la prétention à l'unification inhérente au thème de la raison. Cependant, la défense habermassienne de la modernité et de la raison repose sur le refoulement du thème des limites du langage et de la communication, pourtant constitutif lui aussi de la modernité, et surtout sur la méconnaissance de l'art, tristement réduit, dans la Théorie de l'agir, au statut d'illustration d'une fonction d' « authenticité expressive ». Aux yeux de l'auteur, la mise en question de ces deux aspects suffit à justifier une attitude nuancée à l'égard de toute la construction habermassienne; mais on peut se demander dans quelle mesure il ne se contraint pas alors à revenir à des positions qui, comme il reconnaît lui-même, ont été dépassées par Habermas. 
On peut isoler enfin un dernier ensemble d'études qui visent à apprécier les conclusions habermassiennes en fonction de la situation historique contemporaine qu'elles prétendent assumer. Gérard Raulet conteste ainsi le choix de la communication comme fil conducteur de la théorie sociale. Il amène selon lui à préjuger abusivement de l'unité des phénomènes par-delà la diversité des jeux de langage et des facteurs de différenciation à l'œuvre dans la culture moderne. Il implique d'autre part une vision trop étroite des ressources de la critique : à ramener « la réalité des interactions sociales qui sont la Raison» (p. 103) à la seule figure de l'échange linguistique, ne court-on pas le risque de n'offrir à la théorie sociale qu'une base bien étroite? Cette difficulté est également au cœur du remarquable essai d'Axel Honneth. Celui-ci rappelle que dans sa version horkheimerienne, la théorie critique doit s'appuyer sur une expérience réelle qui de soi implique déjà une tendance à l'émancipation. Mais Habermas n'opère qu'une reprise sélective de cette intention en mettant seulement en valeur la force normative des présuppositions de l'activité communicationnelle (même si l'auteur néglige le fait que, concrètement, Habermas raisonne autant à partir du potentiel présent dans la rationalisation moderne en particulier que des ressources de l'interaction langagière en général). Aujourd'hui, c'est dans la résistance aux atteintes portées aux processus de la reconnaissance sociale (par exemple dans le travail et dans le chômage) qu'il faudrait situer cette origine, plutôt que dans la seule critique des distorsions de la communication, qui n'en forme qu'un moment. Cette proposition d'élargissement ou de rectification de la perspective habermassienne, destinée à rendre possible une meilleure prise en compte des crises contemporaines, conclut de la manière la plus stimulante un recueil particulièrement instructif et, on le voit, riche de suggestions.

Le texte des leçons de 1986 sur le droit et la morale, situées chronologiquement entre la Théorie de l'agir communicationnel et Droit et démocratie constitue, quant à lui, une contribution à la réflexion contemporaine sur l'action ainsi qu'un précieux témoignage sur l'évolution de la pensée de Habermas dans les années qui ont vu la formation de sa pensée politique actuelle. Sous une forme polémique et programmatique, le but de ce court écrit est de montrer qu'il est possible de réinscrire la théorie juridique dans le cadre d'une philosophie pratique sans renoncer au thème d'une pensée critique orientée par la réflexion sur les situations contemporaines.

Pour établir cette thèse, l'auteur engage une contestation du positivisme juridique en reprenant certains thèmes de sa critique de l'interprétation wébérienne de la modernité. Dans la Théorie de l'agir, il se bornait à noter que, historiquement, il était peu vraisemblable que l'autonomisation et la formalisation du droit (seules mises en évidence par le sociologue) aient constitué des phénomènes indépendants et n'aient pas correspondu en particulier à l'apparition de formes de conscience morale élevées, gouvernées par l'exigence de règles impartiales fondées sur l'adhésion rationnelle des intéressés. Ici, l'auteur va plus loin et note par exemple que l'apparition des législations de l'État-providence, significativement dénoncées par Max Weber comme une menace pour la pureté du droit formel parce qu'appuyée sur des impératifs de justice selon lui idéologiques, constitue une sorte de vérification du caractère mythique de l'image d'une légalité détachée de tout besoin de légitimité suggérée par le positivisme juridique. A aucun des moments de son histoire, et à l'époque contemporaine moins que jamais, le droit moderne n'a répondu à l'image 
idéalisée que Weber, prisonnier de son scepticisme dans le domaine pratique, a voulu en donner. Son développement s'est au contraire accompagné - bien sûr sans avoir toujours su la réaliser empiriquement — de l'avènement d'une manière de fonder rationnellement les normes dont l'explicitation autorise aujourd'hui à penser la possibilité d'une interdépendance et d'une interpénétration de la morale et du droit, et donc aussi le sauvetage du noyau rationnel des théories du droit naturel.

Cependant, on pourrait reprocher à cette conclusion son idéalisme. La difficulté provient ici surtout du fait que des sociologues tels que Niklas Luhmann ont développé une image de la société moderne comme d'un ensemble de sous-systèmes indépendants les uns des autres, ce qui, d'une part, interdit au droit l'ambition d'avoir une prise sur l'ensemble social et, d'autre part, laisse penser que la complexité de nos sociétés est telle qu'aucun genre unique de principes, éthiques en particulier, ne peut prétendre y opérer efficacement. Renouant avec la philosophie politique classique au terme d'une critique attentive des hypothèses proposées par certains sociologues et juristes de notre temps, Habermas réaffirme au contraire avec force la capacité qu'a, aujourd'hui encore, un «État de droit » de maîtriser politiquement cette complexité, en organisant des procédures démocratiques destinées à fonder les décisions collectives et à rendre possible l'exercice de la justice. Une norme se trouve ainsi élaborée, qui permet d'évaluer de façon critique les pratiques sociales et politiques existantes.

Du point de vue de l'histoire du développement de la pensée de l'auteur maintenant, cet écrit tend d'abord à dissiper le soupçon légitime selon lequel celui-ci aurait pratiqué, dans les années quatre-vingts, une sorte de fuite en avant dans la théorie morale, le dispensant de rectifier les insuffisances de sa théorie sociale ou de l'adapter aux nouvelles conditions historiques. Il montre aussi la précocité relative de sa rupture avec l'antijuridisme et l'anarchisme qui semblaient impliqués par le diagnostic sur le contemporain proposé dans la Théorie de l'agir, de même que l'importance qu'a eue d'emblée pour Habermas la dualité kantienne du droit et de la morale pour faire obstacle au moralisme auquel risquait de conduire l'adoption de la problématique de l'éthique de la discussion ${ }^{26}$. Mais ces évolutions ne sont pas non plus achevées au moment de la rédaction de l'ouvrage. Ainsi, ne trouve-t-on pas dans Droit et morale, dans lequel les deux termes semblent en équilibre, l'équivalent des pages très décidées de Droit et démocratie où Habermas fait du droit au détriment de la morale, le moyen privilégié d'effectuation historique de la rationalité pratique, ce par quoi elle a vraiment prise sur le réel (cf. cependant la p. 50, qui annonce ce thème). De même, la valorisation un peu terne de l'État de droit (p. 90-92) qui termine les leçons de 1986, même si elle n'en néglige pas les aspects démocratiques, contraste avec la fermeté que mettra Habermas six ans plus tard, à établir la nécessité d'une conjonction entre le libéralisme moderne et la tradition "républicaine » (la liberté des Anciens selon Benjamin Constant) afin de faire de la démocratie politique le pivot de sa théorie juridique. Tout cela fait de Droit et morale, à certains égards, un ouvrage de découverte et de transition, mais qui conserve un intérêt

26. L'impression qu'un tel risque existe effectivement pouvait ressortir des deux ouvrages publiés ultérieurement par Habermas dans ce domaine et traduits en français sous les titres de Morale et communication, Paris, Cerf, 1986, et De l'éthique de la discussion, trad. de l'allemand par Mark HunYADI, Paris, Cerf (« Passages»), 1992. 
propre en raison du sérieux avec lequel y sont pensées les conditions d'une réactivation du projet kantien d'une philosophie pratique qui se lierait avec les acquis des sciences sociales et avec la prise en compte des exigences du présent.

Stéphane HABER

Giulia Sissa, Le Plaisir et le mal. Philosophie de la drogue. Paris, Odile Jacob, 1997. $14,5 \times 22,225$ p., index.

Dans Le Plaisir et le mal, Giulia Sissa fait se croiser, entre autres et dans le désordre, Platon, William Burroughs, Freud et Christiane F. (droguée et prostituée) afin d'obtenir de leurs témoignages la matière d'une réflexion sur la toxicomanie comme forme extrême et excessive du désir et du plaisir. L'auteur entend s'intéresser à la drogue comme à l'objet d'une impossible satisfaction dont il conviendra à terme de montrer qu'elle n'est ni le seul ni le plus convenable paradigme du désir. Entre le témoignage du plaisir excessif (jusques et y compris dans la douleur) que l'auteur trouve chez de Quincey ou Burroughs et l'invitation finale faite au lecteur de «se plier au principe de réalité : pour jouir, il faut faire un effort. Allez donc séduire des hommes, conquérir des femmes, gagner de l'argent, écrire un livre [...] » (p. 190), G. Sissa commente pour les opposer deux théories distinctes du désir et du plaisir : celles de Platon puis de Freud. Le premier donnerait à la philosophie de la drogue son expression la plus «extrême » (la condamnation du désir insatiable), quand le second lui apporterait l'indispensable nuance d'une théorie du plaisir négatif mais du désir satisfait. De ces deux lectures, l'auteur conclura finalement : « $\mathrm{Si}$ Platon avait raison, tout désir fonctionnerait comme une toxicomanie. Si la toxicomanie exhibait la vérité du désir humain, comme le prétend William Burroughs, alors Platon serait notre philosophe le plus clairvoyant. Mais il n'en est rien [...] Il est tentant de jouer avec l'idée que les drogues seraient le paradigme de tous les objets que l'on convoite ou achète. La psychanalyse cependant, et plus précisément Freud, nous en détourne. La centralité du non déplaisir se conjugue ici avec une théorie du désir non insatiable. La satisfaction est possible » (p. 189). Platon joue donc ici le rôle de contempteur du corps. G. Sissa s'appuie sur les formules et les images les plus frappantes de la condamnation philosophique de l'hédonisme (notamment celles de Socrate s'opposant à Calliclès dans le Gorgias), pour montrer que le gouffre sans fond et le tonneau percé sont les caractéristiques mêmes du rapport que le corps entretient à l'égard de son propre plaisir : c'est un trou qui désire sans cesse se boucher, mais la chose est impossible (bouché, un trou ne serait plus un trou). Il faut ainsi se rendre à l'évidence que « le corps platonicien est un corps baroque, déliquescent et fluide. C'est également un corps toxicomane. Arraché à sa dissolution par un remplissage cyclique, il n'est pas, il devient. Tant que le flux l'inonde et se maintient à l'intérieur, on peut faire autre chose que l'abreuver » (p. 53). Ce type de remarques, comme la lecture dont il se soutient, présente, du point de vue de la doctrine platonicienne, le double inconvénient de passer outre tout ce que cette doctrine peut dire du corps (de sa nature, de son soin et de ses apti- 
tudes), mais encore de ne pas tenir compte de l'effort qu'elle conduit, précisément au chapitre des plaisirs, afin de considérer le rapport à soi comme une forme de maîtrise et de labeur, que Michel Foucault désignait dans l'Histoire de la sexualité comme «subjectivation». G. Sissa dit vouloir se distinguer de Foucault en montrant que la thématique de la maîtrise ne rend pas suffisamment compte du caractère insatiable du plaisir. Mais elle le fait en négligeant ce que l'Histoire de la sexualité observait : le soin du corps et le rapport qu'il convient d'adopter à l'égard des plaisirs corporels sont, dans la philosophie platonicienne, indissociables d'une éducation aux plaisirs comme à leur maîtrise. La modération que défend cette philosophie, lorsqu'elle montre combien il est nécessaire de connaître les plaisirs, de les examiner pour mieux en user ou mieux les associer, ne ressemble guère au diagnostic sévère que lui attribue G. Sissa. Ainsi, l'idée que "Platon vise à exclure une fois pour toutes les réjouissances non intellectuelles du genre de vie du savant »(p. 62) paraîtra sans doute incongrue au lecteur du Banquet ou à celui qui entend Socrate dans le Gorgias : « Moi, j'aime Alcibiade, fils de Clinias, et la philosophie. » Vouloir encore, à l'encontre de l'anthropologie du Gorgias (ou du Timée) comme de la psychologie de la République (qui s'efforce précisément de concevoir une éducation des désirs et des plaisirs), reconduire le dialogue platonicien à l'éloge exclusif du plaisir philosophique (que l'auteur réduit, semble-t-il, au plaisir de la lecture ou aux joies du raisonnement), ne peut bien sûr donner lieu qu'à la conclusion que les désirs corporels et le désir de vérité sont séparés par un abîme (p. 92). Mais c'est une conclusion qui n'a pour elle, à notre connaissance, aucune autorité dans le texte platonicien.

De Platon pourfendeur du plaisir impossible, G. Sissa en vient rapidement à Freud, analyste du plaisir négatif et du «désir satiable » (p. 168). Elle montre très clairement comment ce dernier conçoit désir et plaisir sur le modèle d'investissements et d'équilibres énergétiques, pour insister ensuite sur l'expérience freudienne de la cocaïne et son rôle dans la distinction qu'opère finalement Freud entre le plaisir et la mort, la jouissance et l'extinction. Quoiqu'en pensent Lacan et les «psychanalystes lacaniens ", le désir peut rencontrer son objet, atteindre la quiétude que Freud avait su trouver dans cette cocaïne qu'il prenait pour un analgésique (p. 178180). Platon s'était donc bien trompé, désirer et éprouver du plaisir sont des choses possibles et, dans certaines limites, souhaitables. Le Plaisir et le mal s'en tient à cette conclusion, que son auteur regarde comme un élément important de la réflexion sur la dépendance toxicomaniaque; au drogué qui sait que son envie ne cesse jamais, on devrait pouvoir rappeler qu' « un besoin qui monopolise votre temps n'est pas bon, car votre temps c'est votre vie, votre vie c'est vous » (p. 190). Outre cette leçon édifiante, la «philosophie de la drogue » de G. Sissa se prononce de façon assez allusive pour le développement d'une politique publique d'aide aux toxicomanes ( « discours de compréhension ») tout en soulignant l'ambiguité de l'actuelle «complaisance esthétique» (notamment cinématographique) pour la figure du drogué. Mais elle n'examine pas les éventuels effets de cette politique sur l'objet de son essai, le plaisir (du drogué). Dans le même sens et d'emblée, une « philosophie de la drogue » qui ne s'intéresse ni à l'histoire, ni à la nature, ni aux pratiques, ni aux formes et aux coûts sociaux de la drogue parait vouée à l'échec. La seule consultation des études informées sur la consommation des drogues illégales pouvait éventuellement permettre de nuancer le constat selon lequel le drogué poursuivrait sans fin son plaisir; le gouffre ou le panier percé sont des images qui ne 
conviennent guère aux équilibres, certes instables, de la toxicomanie. Les politiques publiques répressives, qui procèdent finalement d'une hypothèse quantitative semblable (en cherchant à contraindre les toxicomanes à diminuer leur consommation), échouent aussi bien aux États-Unis qu'en Europe (particulièrement en France; sur le sujet, cf. la très précieuse étude de Pierre Kopp, L'Économie de la drogue, Paris, La Découverte, 1997). Comme y insistait Gilles Deleuze il y a quelques années, le drogué est aussi le perpétuel désintoxiqué dont l'existence oscille entre la consommation et l'abstinence (dans le même sens, cf. encore l'étude de P. Kopp), incapable d'avoir un usage expérimental ou varié des stupéfiants (intervention dans la « Rencontre au centre Marmottan » avec F. Châtelet, F. Guattari, le $D^{r}$ Claude Olievenstein et al., publiée par la Bibliothèque des mots perdus, 1978). Le Plaisir et le mal, faute d'information, n'atteint ni cette sorte de nuances (la différence entre l'usage de stupéfiants et la toxicomanie aurait pu par exemple trouver quelque aliment dans les Lois de Platon qui font un éloge déterminant de l'ivresse), ni finalement l'objet éthique de son intitulé.

Jean-François Pradeau

Hervé Barreau, Le Temps. Paris, Presses universitaires de France, 1996. 11,5 × 17,5, 128 p., bibliogr. (« Que sais-je? », 3180).

L'ouvrage se divise en deux parties : « Le temps dans la vie quotidienne » et « Le temps dans la connaissance scientifique ». L'auteur décline chacun de ces temps et s'interroge sur leurs différences.

Le temps biologique est perçu à travers les rythmes auxquels l'organisme s'adapte et qu'il est susceptible de répéter, le temps physiologique à travers les âges de la vie et le vieillissement. Les notions de temps et de durée évoluent de l'enfant à l'adulte, forgées par la langue qui véhicule « toute une sédimentation de la connaissance commune du temps ». Le rythme de la vie individuelle doit se soumettre à celui de la vie sociale, lui-même régulé par celui du monde. Cet emboîtement des rythmes finit par constituer notre représentation du temps. Le calendrier joue à cet effet un rôle important, et l'auteur en retrace l'histoire technique.

Ce temps des calendriers débouche sur le temps scientifique, avec l'horlogerie scientifique moderne et la datation des longues durées. Une introduction succincte mais claire aux temps de la théorie de la relativité et de la mécanique quantique complète le tableau.

L'auteur a donc su faire passer de grands moments de l'histoire de la mesure du temps et en montrer les conséquences sur la vie humaine ou sur la connaissance du monde. De Jean Piaget à Gaston Berger, du calendrier égypto-julio-grégorien aux étalons à césium en passant par le pendule de Huyghens, de Galilée à Erwin Schrödinger en passant par Maxwell et Einstein, l'expérience humaine du temps suit de près l'évolution des sciences et des techniques.

$\mathrm{Au}$ chapitre des regrets, on peut objecter l'absence de référence à la théorie des systèmes dynamiques, la «mathématique du temps » pour reprendre le titre d'un 
ouvrage de Steven Smale, avec les notions d'attracteurs ou de « catastrophes », si présentes en physique, en économie, ou en chronobiologie qui est pourtant mentionnée (voir, par exemple, Ivar EKELAND, Le Calcul, l'imprévu. Les figures du temps de Kepler à Thom, Paris, Seuil, "Science ouverte », 1984). De même, le temps de l'évolution des espèces n'est pas banal, avec ses débats autour de la sélection naturelle et du neutralisme, ou entre gradualisme et " équilibres ponctués ». Le temps de l'Histoire, malgré de timides allusions, est curieusement absent de l'ouvrage, bien que ce sujet ait été très débattu au moins depuis les Annales (par exemple, « $\mathrm{La}$ Longue Durée » de Fernand Braudel, Annales. ESC, 4, 1958, ou L'Ordre du temps de Krzysztof Pomian, Paris, Gallimard, 1984, ou Le Futur passé de Reinhart Koselleck, Paris, École des hautes études en sciences sociales, 1990, etc.).

L'ouvrage n'en demeure pas moins une bonne invitation à réfléchir sur le sujet difficile du temps. Un titre plus précis et plus restrictif aurait peut-être permis de ne pas conduire les lecteurs et les lectrices vers l'idée que le temps se limite aux seuls aspects abordés.

Noël BonNEUIL

Du film ethnographique à l'anthropologie filmique. Textes rassemblés et présentés par Claudine de FranCE. Paris, Éd. des Archives contemporaines, 1995. 15,5 × $23,168 \mathrm{p}$.

Si le recours à l'image est aussi ancien que le savoir anthropologique moderne, on assiste aujourd'hui à un déplacement du modèle des films spécialisés de la référence au cinéma documentaire vers le souci de l'investigation scientifique. Cet ouvrage marque un tel mouvement en proposant six textes rassemblés et présentés par Claudine de France, responsable de la formation doctorale anthropologique et cinématographique à l'Université de Nanterre.

Dans son article de présentation, C. de France affirme les objectifs et les ambitions d'une anthropologie filmique. Elle pose la question du passage de l' « accumulation primitive » de films ethnographiques à la constitution d'une véritable discipline, projet à l'origine de l'ouvrage. Cette anthropologie filmique apparaît alors comme «l'étude de l'homme filmable - susceptible d'être filmé - mais également celle de l'homme filmé, tel qu'il apparaît mis en scène par le film ».(p. 7). Le second terme de cette définition renvoie aux contraintes techniques de l'acte de filmer et non pas à une réflexion sur la présentation de soi, voire sa représentation, familière pourtant aux sociologues.

C. de France n'ignore pas la difficulté qu'il y a à définir un objet de recherche partant de l'outil de sa restitution. Mais elle n'échappe pas à la pétition de principe quand elle justifie cette entreprise par les contraintes propres au travail filmique. Les renouvellements des techniques audiovisuelles, le faible recours des chercheurs à leur usage et la rareté de travail critique et épistémologique sur ces genres de productions sont autant de raisons de saluer la détermination des anthropologues cinéastes et leur intention de transmettre et de réfléchir les savoir-faire issus de leurs 
expériences. Faut-il pour autant assujettir l'autonomie de la construction d'un objet anthropologique - ou de tout autre objet de sciences sociales - à une technique récente et aujourd'hui indispensable? On peut en douter après l'acte de fondation durkheimienne de la sociologie qui est aussi un acte de rupture à l'égard des apparentes spécificités de la méthode statistique de son temps.

Un second argument vient à l'appui du projet d'anthropologie filmique. L'anthropologie en général a recours aux traditions orales et le film serait adéquat à leur restitution : il reléguerait l'écrit, et restituerait à la parole sa suprématie. L'écrit, dans ces conditions, n'interviendrait plus en amont ou pendant l'enquête, mais en aval. Et cette tradition orale d'un nouveau genre, voulue par C. de France, lui fait aussi craindre le risque du rejet total de l'écrit chez certains anthropologues cinéastes alors même que la jeune discipline aurait tant besoin d'avocats auprès de ses pairs. On n'en finira jamais de plaider les vices ou les vertus de l'écrit et de l'oral tant que l'enregistrement de la parole, sonore ou écrit, tant que les images, fixes ou animées, ne seront pas considérées comme autant de modalités d'objectivation qui forment une panoplie pour l'enquête anthropologique ou sociologique.

Les anthropologues cinéastes revendiquent la particularité de leur démarche face aux spécialistes de l'image animée, notamment les journalistes de documentaires. Ainsi C. de France retient trois critères de distinction à leur égard. Le public du film anthropologique, en premier lieu, serait restreint à la communauté des chercheurs. Mais n'en va-t-il pas, pour les sciences sociales, de l'image comme du livre : l'une et l'autre connaissent des publics en priorité spécialisés mais aussi multiples et dont la commande (qu'elle provienne des producteurs ou de leurs homologues les éditeurs) médiatise les contraintes. Une seconde caractéristique serait l'objectif poursuivi par le chercheur : la description ethnographique (verbale ou non verbale). On peut craindre ici encore une pétition de principe. Le troisième argument est recevable : l'anthropologue cinéaste, contrairement au journaliste, travaille en ayant la faculté de prendre son temps et de jouer sur l'espace. Ce point est en effet essentiel. Il est même parfois identifié par les documentaristes les plus scrupuleux. Il peut de plus rencontrer le souci expérimental qui s'exprime dans la réflexion actuelle sur les sciences sociales ${ }^{27}$. Pour d'autres raisons encore, les anthropologues cinéastes rencontrent une donnée qu'on retrouve dans l'ensemble des sciences sociales : l'autonomie de la pratique de l'image est faible du fait de la monopolisation par les journalistes des formes légitimes de restitution des phénomènes de société ${ }^{28}$.

La portée de l'anthropologie filmique revendiquée par les auteurs est démontrée efficacement dans les articles de Jane Guéronnet, « Ritualité et coopération dans une

27. Voir Les Formes de l'expérience. Une nouvelle histoire sociale, dir. Bernard LEPETIT, Paris, Albin Michel, 1995 ou Jeux d'échelles. De la micro-analyse à l'expérience, dir. Jacques Revel, Paris, Gallimard/Seuil, 1996.

28. La meilleure illustration en est l'ouvrage La Misère du monde, dir. par Pierre BourDIEU, Paris, Seuil, 1993, où la revendication de la pratique sociologique proprement savante ne s'exerce plus à l'égard d'une sociologie routinisée (comme dans Pierre BourdiEu, Jean-Claude Passeron, Jean-Claude Chamboredon, Le Métier de sociologue, Paris/La Haye, Mouton, 1968) mais contre les formes journalistiques de représentation des phénomènes sociaux. Le succès du livre provient précisément du fait qu'il se situe sur cette frontière, comme le montre depuis la multiplication dans le quotidien Libération de tranches de vie à la manière de la " Misère du monde ». 
technique du Corps ", et de Annie Comolli, "L'investigation filmique des apprentissages ». Prolongeant la tradition maussienne de recours à l'image pour l'étude des techniques sociales du corps, ces contributions confirment que la caméra est un outil privilégié pour l'étude des manifestations sociales incorporées. Les anthropologues américains de l'entre-deux-guerres, tels Gregory Bateson ou Margaret Mead, l'avaient bien compris. Ils furent parmi les premiers à réaliser des films dans des conditions techniques particulièrement inconfortables comparées à celles d'aujourd'hui. Dans le domaine de la sociologie, l'analyse des rites sociaux d'interactions se prêterait tout particulièrement à l'étude par le film. Erving Goffman luimême eut recours à l'analyse d'images, mais publicitaires, dans son étude de la représentation du genre féminin ${ }^{29}$. Bien que cette recherche n'ait pas ouvert de tradition en sociologie, nous sommes bien aujourd'hui au point où les sciences sociales dans leur généralité peuvent bénéficier de l'expérience des anthropologues cinéastes. Les textes de Jean-Marc Rosenfeld, de Philippe Lourdou et l'entretien accordé par Jean Rouch fournissent alors au lecteur des réflexions et des témoignages de la première importance à propos de la manière de filmer et du travail de commentaire.

Le principal intérêt de l'ouvrage est de mettre en évidence que si la production d'images occupe une place importante au sein de notre société, les chercheurs en sciences sociales n'en sont qu'aux balbutiements de sa pratique empirique. La consultation des bibliographies de films spécialisés (fournies dans ce livre systématiquement à la manière des bibliographies d'imprimés), conforte la conviction que le genre demeure l'exception non seulement parce qu'il est peu pratiqué mais encore du fait des difficultés que les chercheurs peuvent rencontrer pour prendre connaissance des travaux réalisés.

Le projet d'anthropologie filmique marque bien un moment de renouveau des sciences sociales actuelles. Mais, pris aujourd'hui entre trois genres antérieurs, l'enquête anthropologique, le cinéma ethnographique, et le documentaire de presse audiovisuelle, il reste dépendant d'une revendication de spécificité qui s'exprime par la mise à l'écart de celui de ces trois genres le moins culturellement légitime (le journalisme), le souci de dépasser sa référence à une pratique artistique (le cinéma), et la tentative de subvertir la discipline scientifique mère (l'anthropologie). Reste aujourd'hui à intégrer pleinement la pratique filmique dans la panoplie des sciences sociales en en comparant systématiquement les ressources et les effets avec les procédés plus traditionnels (entretiens, statistiques, sources imprimés secondaires). Dès lors, une critique en terme de construction d'objet ou d'élaboration expérimentale ferait réévaluer ce que les réalisateurs de documentaires peuvent mesurer s'ils partagent un souci de connaissance: l'importance du jeu sur le temps accordé à l'enquête, ou encore le travail de construction qui s'opère au cadrage ou bien au montage.

Marie JAISSON

29. Erving Goffman, Gender Advertisements, New York, Harper Colophon Books, 1976. 